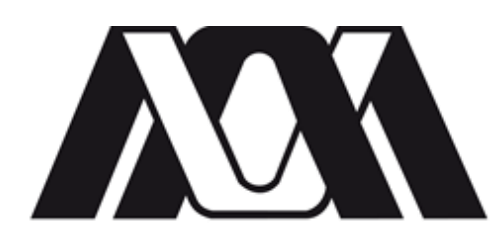

Casa abierta al tiempo

UNIVERSIDAD AUTÓNOMA METROPOLITANA

UNIDAD IZTAPALAPA

\title{
Efectos de la variación simultánea de la frecuencia respiratoria y el volumen corriente sobre la actividad autonómica cardiovascular
}

\author{
Tesis que presenta \\ Alejandra Guillén Mandujano \\ Para la obtención del grado de \\ Doctora en Ciencias (Ingeniería Biomédica)
}

Asesor:

Dr. Salvador Carrasco Sosa

Comité Evaluador:

Presidente: Dra. Claudia Lerma González

Secretario: Dr. Juan Carlos Echeverría Arjonilla

Vocal: $\quad$ Dra. Guadalupe Dorantes Méndez

Vocal: Dr. Miguel Ángel Peña Castillo

Vocal: Dr. Salvador Carrasco Sosa 


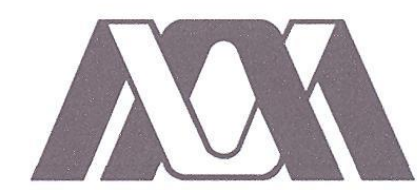

Casa abierta al tiempo

UNIVERSIDAD AUTÓNOMA METROPOLITANA

UNIDAD IZTAPALAPA

\title{
Efectos de la variación simultánea de la frecuencia respiratoria y el volumen corriente sobre la actividad autonómica cardiovascular
}

\author{
Tesis que presenta \\ Alejandra Guillén Mandujano \\ Para la obtención del grado de \\ Doctora en Ciencias (Ingeniería Biomédica)
}

Asesor:

Dr. Salvador Carrasco Sosa

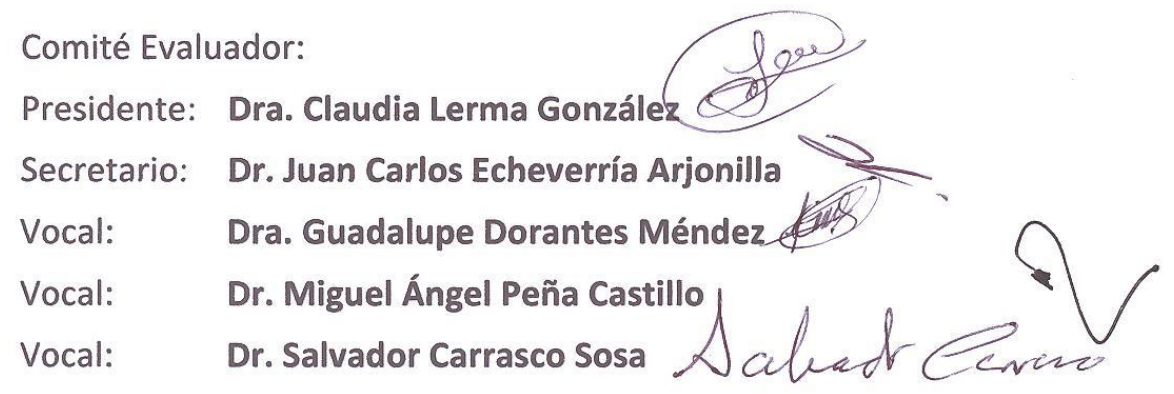




\section{Agradecimientos}

Este proyecto pudo llevarse a cabo gracias al apoyo de:

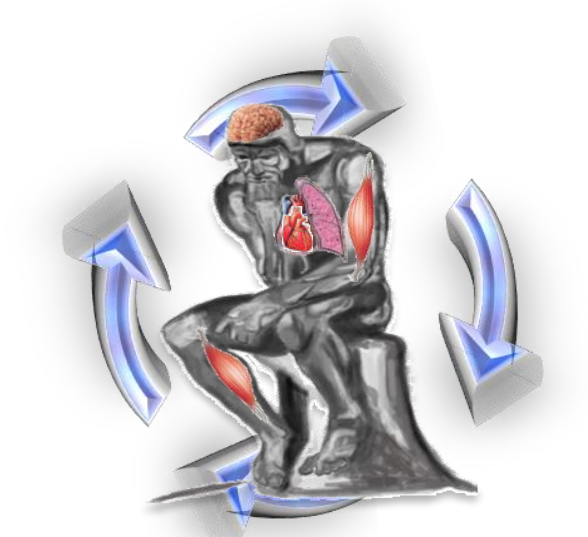

Pensar y Hacer Fisiología

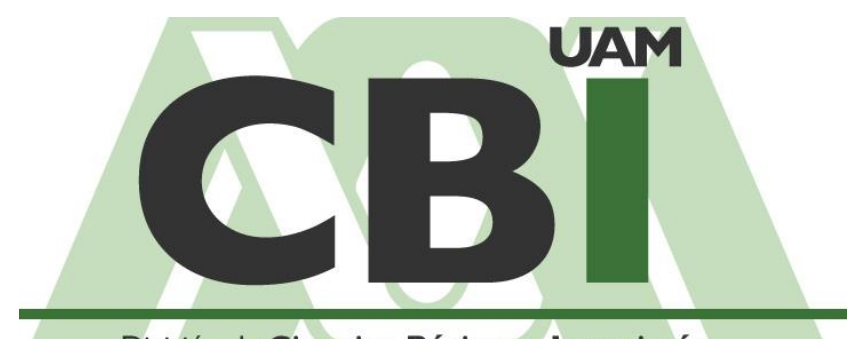

División de Ciencias Básicas e Ingeniería
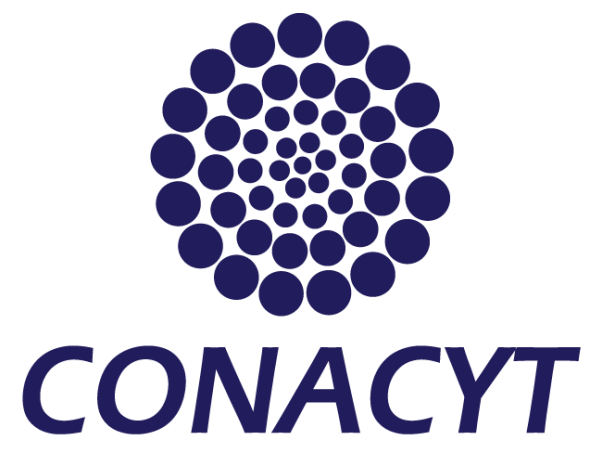

Consejo Nacional de Ciencia y Tecnología
Laboratorio de Fisiología Médica

Departamento Ciencias de la Salud

División de Ciencias Biológicas y de la Salud.

UAM Iztapalapa

División de Ciencias Básicas e

Ingeniería

UAM Iztapalapa

Programa Nacional de Posgrados de Calidad CONACyT

CVU: 168495

Beca No.: 28593 


\section{Índice}

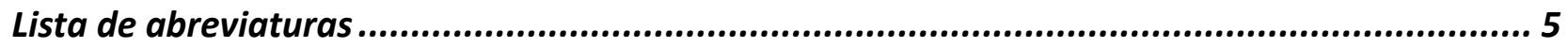

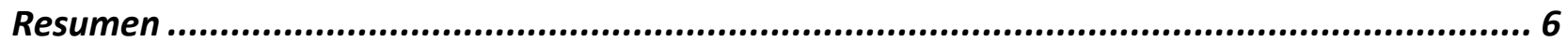

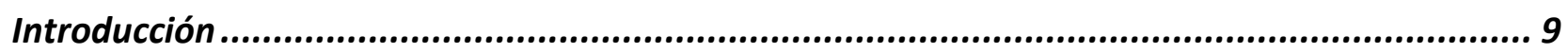

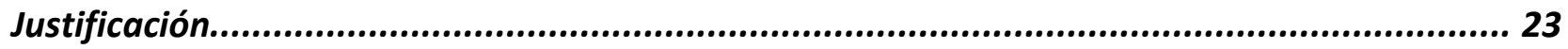

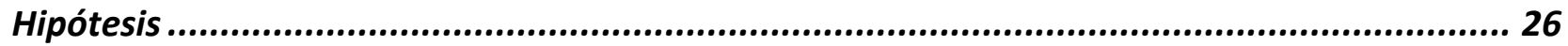

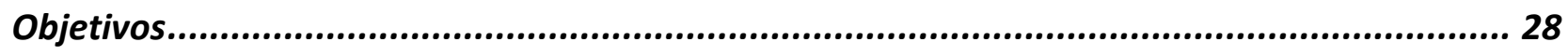

Etapa I: Pruebas piloto para el desarrollo de la metodología............................................ 31

Etapa II: Aplicación experimental de la metodología desarrollada...................................... 42

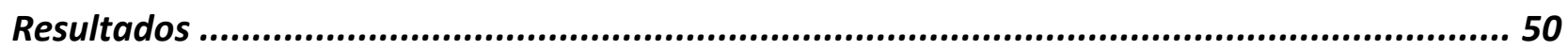

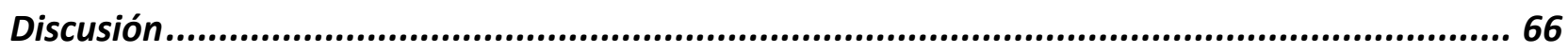

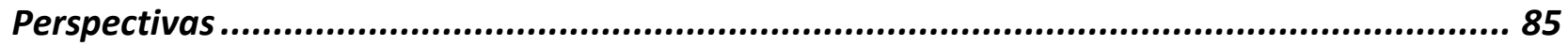

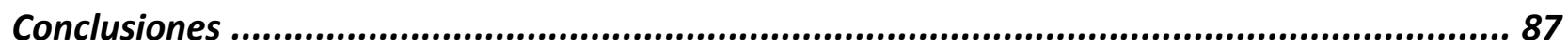

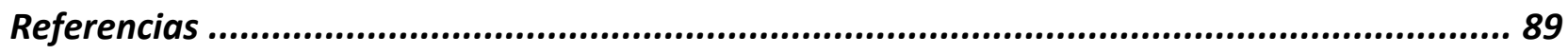

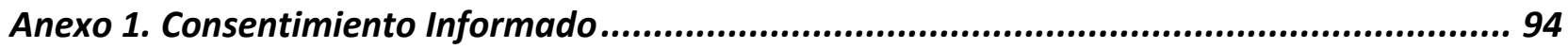

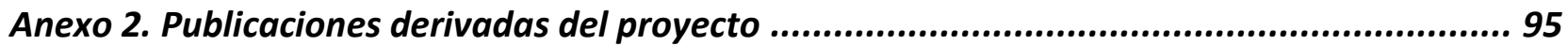




\section{Lista de abreviaturas}

\begin{tabular}{|c|c|}
\hline $\mathrm{AF}_{\mathrm{RR}}$ & Componente de alta frecuencia de la variabilidad de la frecuencia cardiaca \\
\hline ASR & Arritmia sinusal respiratoria \\
\hline $\mathrm{BF}_{\mathrm{RR}}$ & Componente de baja frecuencia de la variabilidad de la frecuencia cardiaca \\
\hline $\mathrm{CC}$ & Comando central \\
\hline CPA & Cambio de postura activo \\
\hline DC & Demodulación compleja \\
\hline DEC & Decremental \\
\hline DPWVS & Distribución Pseudo Wigner-Villé suavizada \\
\hline DTF & Distribución tiempo-frecuencia \\
\hline ECG & Electrocardiograma \\
\hline $\mathrm{E}_{\mathrm{FR}}$ & Efectos autonómico-cardiovasculares de la variación de la frecuencia respiratoria \\
\hline $\mathrm{ETCO}_{2}$ & Fracción espiratoria de bióxido de carbono \\
\hline $\mathrm{E}_{\mathrm{VC}}$ & Efectos autonómico-cardiovasculares de la variación del volumen corriente \\
\hline $\mathrm{E}_{\mathrm{VC}+\mathrm{FR}}$ & $\begin{array}{l}\text { Efectos autonómico-cardiovasculares de la variación simultánea de la frecuencia } \\
\text { respiratoria y el volumen corriente }\end{array}$ \\
\hline $\mathrm{FC}_{\mathrm{m}}$ & Frecuencia cardiaca media \\
\hline FCVD & Frecuencia respiratoria constante, volumen corriente decrementado \\
\hline FCVI & Frecuencia respiratoria constante, volumen corriente incrementada \\
\hline FDVC & Frecuencia respiratoria decrementada, volumen corriente constante \\
\hline FDVD & Frecuencia respiratoria decrementada, volumen corriente decrementado \\
\hline FDVI & Frecuencia respiratoria decrementada, volumen corriente incrementado \\
\hline FIAF $_{R R}$ & $\begin{array}{l}\text { Frecuencia instantánea del componente de alta frecuencia de la variabilidad de la } \\
\text { frecuencia respiratoria }\end{array}$ \\
\hline FIVC & Frecuencia respiratoria incrementada, volumen corriente constante \\
\hline FIVD & Frecuencia respiratoria incrementada, volumen corriente decrementada \\
\hline FIVI & Frecuencia respiratoria incrementada, volumen corriente incrementado \\
\hline FR & Frecuencia respiratoria \\
\hline $\mathrm{FR}_{\mathrm{LI}}$ & Frecuencia respiratoria linealmente incrementada \\
\hline $\mathrm{FR}_{\mathrm{LI}} / \mathrm{VC}_{\mathrm{LI}}-\mathrm{VC}_{\mathrm{LD}}$ & \\
\hline II & Índice de interferencia \\
\hline INC & Incremental \\
\hline $\ln \mathrm{AF}_{\mathrm{RR}}$ & $\begin{array}{l}\text { Transformación logarítmica de la potencia del componente de alta frecuencia de la } \\
\text { variabilidad de la frecuencia cardiaca }\end{array}$ \\
\hline MAVT & Modelos autorregresivos variantes en el tiempo \\
\hline MRC2 & Maniobras de respiración controlada doblemente variantes \\
\hline RLS & Estimación por mínimos cuadrados recursiva (Recursive Least Squares) \\
\hline $\mathrm{RR}_{\mathrm{m}}$ & Nivel medio de los intervalos RR \\
\hline $\mathrm{SA}$ & Sinoatrial \\
\hline SNA & Sistema nervioso autónomo \\
\hline $\mathrm{VC}$ & Volumen corriente \\
\hline $\mathrm{VC}_{\mathrm{LD}}$ & Volumen corriente linealmente decrementado \\
\hline $\mathrm{VC}_{\mathrm{LI}}$ & Volumen corriente linealmente incrementado \\
\hline VFC & Variabilidad de la frecuencia cardiaca \\
\hline VP & Ventilación pulmonar \\
\hline$\Delta \mathrm{I}$ & $\begin{array}{l}\text { Diferencia entre interceptos de las regresiones } \ln \mathrm{AF}_{\mathrm{RR}}-\mathrm{VC}_{\mathrm{LI}} \text { y } \ln \mathrm{AF}_{\mathrm{RR}}-\mathrm{VC}_{\mathrm{LD}} \text {, índice de } \\
\text { histéresis }\end{array}$ \\
\hline
\end{tabular}




\section{Resumen}

Introducción. La amplitud de la arritmia sinusal respiratoria (ASR) depende tanto de la frecuencia respiratoria (FR) como del volumen corriente (VC). No obstante, los efectos de cada variable respiratoria han sido establecidos por separado, manteniendo a la otra variable constante, y empleando protocolos con pocos valores elegidos aleatoriamente y administrados de manera discontinua. Además, hay muy pocos estudios sobre el efecto del VC sobre la amplitud de la ASR y aún menos comparaciones entre sexos.

Objetivo. Evaluar, en hombres y mujeres jóvenes y sanos, los efectos interactivos de la variación simultánea y lineal de la FR y el VC sobre dos índices confiables de la ASR, la potencia de alta frecuencia de los intervalos $\mathrm{RR}\left(\mathrm{AF}_{\mathrm{RR}}\right)$ y el nivel medio de los intervalos $\mathrm{RR}\left(\mathrm{RR}_{\mathrm{m}}\right)$.

Método. Se registraron el electrocardiograma (ECG), el VC y el perfil espiratorio de $\mathrm{CO}_{2}$ de 18 mujeres y 20 hombres voluntarios jóvenes (entre 18 y 25 años de edad), sanos (ECG normal, normotensos, espirometría normal, sin adicciones) durante la ejecución, guiada visualmente, de 3 maniobras respiratorias de 150 ó 30 s de duración cada una en postura sentada: FR linealmente incrementada $\left(\mathrm{FR}_{\mathrm{LI}}\right)$ de 0.15 a $0.5 \mathrm{~Hz}$ a VC constante de $1 \mathrm{~L}$ para hombres y $0.8 \mathrm{~L}$ para mujeres; VC linealmente incrementado respiración por respiración $\left(\mathrm{VC}_{\mathrm{LI}}\right)$ seguido de $\mathrm{VC}$ linealmente disminuido $\left(\mathrm{VC}_{\mathrm{LD}}\right)$ en rangos de 1 a $2.5 \mathrm{~L}$ para hombre y de 0.8 a $2.0 \mathrm{~L}$ para mujeres, a FR constante de $0.2 \mathrm{~Hz}$; y la aplicación simultánea de $\mathrm{FR}_{\mathrm{LI}}$ y $\mathrm{VC}_{\mathrm{LI}}-\mathrm{VC}_{\mathrm{LD}}$. Los protocolos de $30 \mathrm{~s}$ se repitieron en postura erguida. Los espectros tiempo-frecuencia de las series de los intervalos RR y de la respiración fueron estimados mediante la distribución pseudo-Wigner-Villé suavizada, para estimar la potencia de alta frecuencia de la respiración y la potencia de $\mathrm{AF}_{\mathrm{RR}}$. Se analizaron los efectos de las maniobras mediante el cálculo de las diferencias control-maniobra de los valores medios y la comparación estadística de los parámetros derivados del análisis de correlación lineal entre los indicadores de la ASR y las variables respiratorias.

Resultados. Las relaciones $\mathrm{AF}_{\mathrm{RR}}-\mathrm{FR}$ y $\mathrm{AF}_{\mathrm{RR}}-\mathrm{VC}$ fueron similares entre géneros. Las diferencias control-maniobra de $\mathrm{AF}_{\mathrm{RR}}$ y $\mathrm{RR}_{\mathrm{m}}$ durante la maniobra combinada fueron aproximadamente iguales a la suma de aquéllas correspondientes a las maniobras independientes. Los coeficientes de 
correlación entre $\mathrm{FR}_{\mathrm{LI}} \mathrm{y} \mathrm{AF}_{\mathrm{RR}}$ por separado y en combinación fueron mayores a 0.90 y negativos, con mayores pendientes durante la maniobra combinada $(\mathrm{p}<0.001)$. La pendiente de la regresión $\mathrm{FR}_{\mathrm{LI}}-\mathrm{AF}_{\mathrm{RR}}$ aumentó casi al doble en la maniobra combinada $(\mathrm{p}<0.001)$. Las relaciones $\mathrm{AF}_{\mathrm{RR}}-\mathrm{VC}_{\mathrm{LI}}$ y $\mathrm{AF}_{\mathrm{RR}}-\mathrm{VC}_{\mathrm{LD}}$ presentaron, en las maniobras independientes, tres combinaciones de pendientes con diferente signo, todas con histéresis: en $49 \%$ de los registros la relación $\mathrm{AF}_{\mathrm{RR}}-\mathrm{VC}_{\mathrm{LI}}$ fue negativa y la relación $\mathrm{AF}_{\mathrm{RR}}-\mathrm{VC}_{\mathrm{LD}}$ fue positiva, con pendientes e interceptos significativamente diferentes $(\mathrm{p}<0.001)$; en $28 \%$ ambas relaciones fueron positivas pero con diferentes pendientes e interceptos $(\mathrm{p}<0.01)$ y en el $23 \%$ restante ambas relaciones fueron negativas, con pendientes similares pero interceptos diferentes $(\mathrm{p}<0.001)$. Durante la maniobra combinada, la relación $\mathrm{AF}_{\mathrm{RR}}-\mathrm{VC}_{\mathrm{LI}}$ presentó pendiente negativa y la relación $\mathrm{AF}_{\mathrm{RR}}-\mathrm{VC}_{\mathrm{LD}}$ pendiente positiva, ambas con altos coeficientes de correlación y mayores valores e histéresis que las relaciones independientes. Con respecto a sus valores en la postura sentada, en la postura erguida todas las pendientes de todas las relaciones disminuyeron y presentaron menores coeficientes de correlación $(\mathrm{p}<0.001)$.

Discusión y conclusiones. El novedoso procedimiento usado, consistente en la aplicación de maniobras respiratorias rápidas, no fatigantes, no estacionarias, con variación simultánea de FR y VC, analizadas con una distribución tiempo-frecuencia, permite documentar que:

1) La $\mathrm{FR}_{\mathrm{LI}}$ atenúa consistentemente a la $\mathrm{ASR}$, aplicada por separado o en combinación con cambios en el VC. Sin embargo, la relación entre la FR y la frecuencia instantánea del $A_{\mathrm{RR}}$ presentó un valor umbral que podría explicarse por el fenómeno de aliasing cardiaco (Witte et al. 1988)

2) Los efectos del $\mathrm{VC}_{\mathrm{LI}^{-}} \mathrm{VC}_{\mathrm{LD}}$ por separado son ambiguos y presentan histéresis, ya que:

a. el $\mathrm{VC}_{\mathrm{LI}}$ sólo incrementa la amplitud de la ASR hasta un valor umbral, más allá del cual la ASR no aumenta o disminuye.

b. a un mismo valor de VC, el valor correspondiente de ASR correspondiente a la porción linealmente incrementada es diferente al de la linealmente decrementada.

3) Tanto hombres como mujeres presentaron cambios en la amplitud de la ASR con valores y comportamientos dinámicos similares ante todas las maniobras estudiadas. Estos resultados sugieren que la respuesta del control autonómico cardiovascular a los estímulos respiratorios no se ve afectada por diferencias sexuales ni por cambios en los niveles hormonales. 
4) La combinación de $\mathrm{FR}_{\mathrm{LI}}$ y $\mathrm{VC}_{\mathrm{LI}}-\mathrm{VC}_{\mathrm{LD}}$ induce un efecto atenuante en la amplitud de la $\mathrm{ASR}$ equivalente a la suma de los efectos independientes, indicando que estos estímulos no se interfieren entre sí al modular la actividad autonómica generadora de la ASR. En otras palabras, sugieren que los núcleos autonómicos integran linealmente y por separado las aferencias respiratorias.

5) La superposición de las maniobras de respiración controlada y el cambio de postura mejoró la interpretabilidad de los cambios de la potencia de $\mathrm{AF}_{\mathrm{RR}}$, ya que mantuvo constante la influencia respiratoria sobre los núcleos autonómicos. Estos resultados apoyan aún más la idea de que los núcleos autonómicos son capaces de sumar diversas aferencias para producir una salida proporcional.

Los resultados obtenidos mediante las maniobras de control respiratorio propuestas aportan nueva información que amplía el conocimiento aceptado sobre los mecanismos generadores de la ASR y el funcionamiento de las estructuras nerviosas involucradas. En particular, sugieren que es necesario incorporar al comando central como el responsable de la influencia del control respiratorio voluntario sobre la amplitud de la ASR. El umbral observado al analizar el efecto de la variación lineal creciente del VC sobre la ASR cuestiona a la conducta usual de normalizar la ASR con el VC y enfatiza la importancia de caracterizar la respuesta de cada individuo a los cambios dinámicos de las dos principales variables respiratorias en rangos mayores a los que se utilizan comúnmente. 


\section{Introducción}

La frecuencia cardiaca instantánea, medida como el inverso del intervalo de tiempo que transcurre entre ondas $\mathrm{R}$ sucesivas (RR), varía continuamente a lo largo del tiempo, presentando fluctuaciones latido a latido. Estas variaciones se ponen en evidencia cuando se grafican sus valores a lo largo del tiempo, formando una señal conocida como tacograma (Fig. 1). A esta característica fisiológica de la función cardiaca se le conoce como variabilidad de la frecuencia cardiaca (VFC). Aunque su

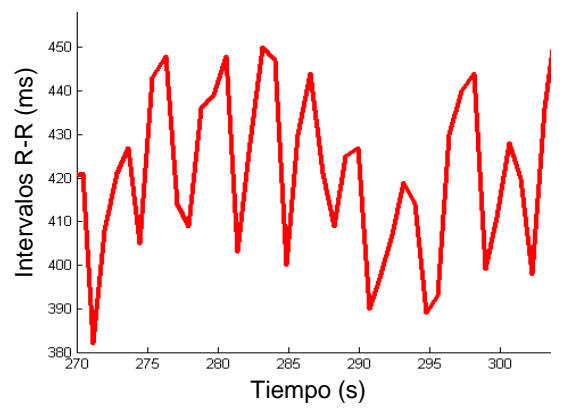

Fig. 1. Ejemplo de un registro de la variación temporal de frecuencia cardiaca instantánea o tacograma.

estudio formal comenzó hace relativamente poco tiempo (Task Force 1996), su utilidad como índice no invasivo del funcionamiento del sistema nervioso autónomo (SNA) la ha convertido en una herramienta de investigación muy utilizada por diversos investigadores en una gran variedad de condiciones de salud y enfermedad.

\section{Fisiología de la VFC}

La función cardiovascular está regulada por un complejo sistema de control que depende de múltiples factores, aunque se destaca la participación del SNA, el cual inerva al corazón y a los vasos sanguíneos. La frecuencia cardiaca está controlada por el nodo sinoatrial (SA), el cual recibe la influencia de las dos ramas del SNA: la simpática, que provoca aceleración en la frecuencia cardiaca y la parasimpática o vagal, que produce diminución de la frecuencia. Una de las técnicas más estudiadas y mejor validadas para extraer información sobre el funcionamiento del SNA a partir del tacograma es el análisis espectral de la VFC (Task Force 1996, Parati et al. 2006). El espectro de la VFC presenta tres componentes principales a los que se les ha asociado correlatos funcionales. La descarga nerviosa vagal es rápida, presenta frecuencias entre 0.15 y $0.4 \mathrm{~Hz}$ generando el componente de alta frecuencia o $\mathrm{AF}_{\mathrm{RR}}$, y está modulada principalmente por la respiración (Brown et al. 1993). La influencia simpática es lenta, con frecuencias entre 0.04 y 0.15 $\mathrm{Hz}$, contribuyendo a la potencia del componente de baja frecuencia $\left(\mathrm{BF}_{\mathrm{RR}}\right)$. Debido a la integración de estas dos influencias, la señal formada al graficar la frecuencia cardiaca latido a latido contra el tiempo es compleja (Fig. 2). Mediante el análisis espectral de la señal de VFC es posible recuperar estos componentes en frecuencia y calcular su potencia y frecuencia central, lo 
que permite estudiar de forma indirecta y no invasiva al SNA y la contribución de cada una de sus ramas al control cardiovascular (Task Force 1996). El indicador espectral cuya correlación fisiológica ha sido claramente demostrada la potencia del componente $\mathrm{AF}_{\mathrm{RR}}$, la cual refleja únicamente la actividad vagal (Malliani et al. 1991, Malliani et al. 1998, Pagani et al. 1986, Parati et al. 2006). El correlato funcional de la potencia del componente $\mathrm{BF}_{\mathrm{RR}}$ es equívoco, ya que parece depender tanto de la modulación simpática como de la vagal (Eckberg 2003, Malliani et al. 1991, Parati et al. 1995).
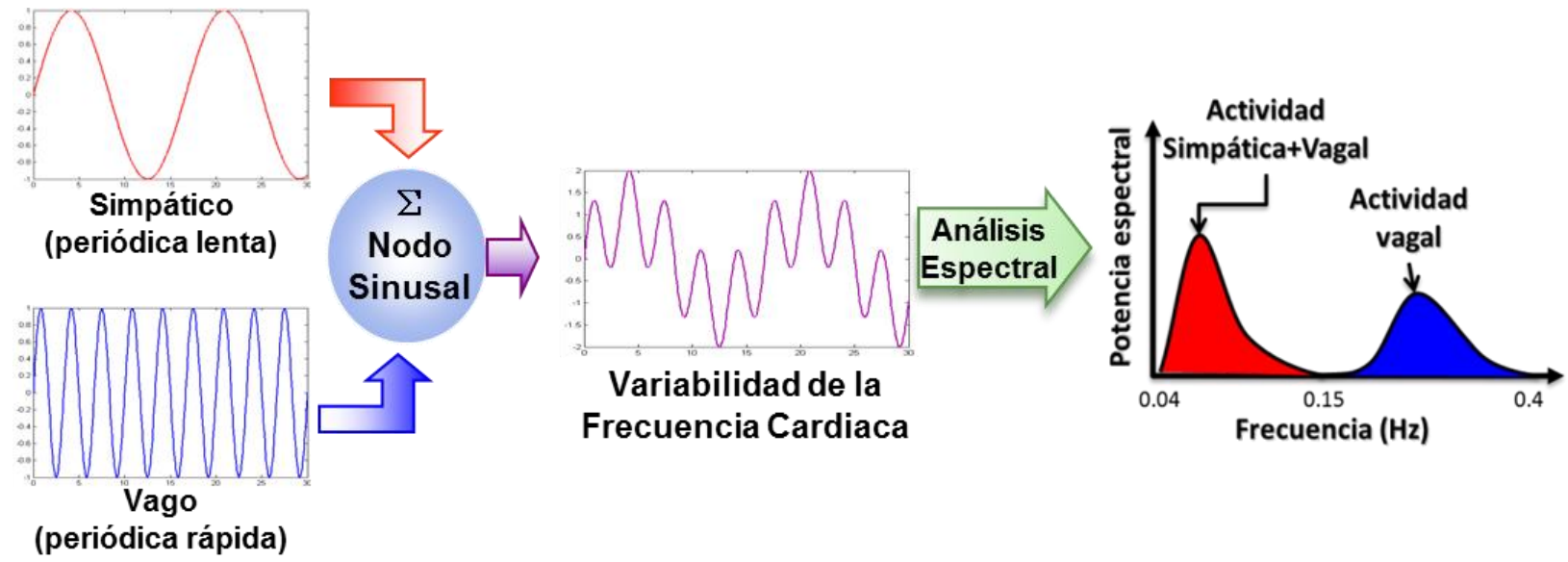

Fig. 2. Representación esquemática del origen de los componentes espectrales de la variabilidad de la frecuencia cardiaca y cómo pueden estimarse mediante análisis espectral.

\section{Técnicas para el análisis espectral de la VFC en condiciones no estacionarias}

Existen numerosas técnicas que permiten el análisis espectral de la VFC. Algunas requieren garantizar una mínima estacionalidad en la señal a evaluar, es decir, que la frecuencia central de los componentes y sus momentos estadísticos se mantengan constantes durante el tiempo considerado en el análisis. Sin embargo, en la mayor parte de las circunstancias fisiológicas y clínicas el análisis espectral estacionario resulta insuficiente, puesto que las características de las señales cardiovasculares $\mathrm{y}$, por lo tanto, de sus componentes en frecuencia, cambian constantemente a lo largo del tiempo, ya sea abrupta o suavemente. Para poder obtener información confiable de estas señales no estacionarias se han desarrollado diversas herramientas capaces de estimar cómo evolucionan las potencias y frecuencias de los componentes a lo largo del tiempo. Cada una presenta ventajas y desventajas que deben ser consideradas al momento de seleccionar alguna para el análisis de un conjunto de datos en particular (Mainardi et al. 2009) 
Las metodologías de análisis espectral tiempo-frecuencia o variantes en el tiempo que han sido más utilizadas para el estudio de la VFC en condiciones no estacionarias son: las distribuciones tiempo-frecuencia (DTF), en especial la distribución pseudo-Wigner-Villé suavizada (DPWVS); los modelos autorregresivos variantes en el tiempo (MAVT) y la demodulación compleja (DC).

\section{Distribución Pseudo Wigner-Villé Suavizada}

Las DTF de la llamada clase de Cohen son transformaciones matemáticas que permiten estimar la contribución en potencia y la frecuencia central de los diferentes componentes espectrales de una señal en un instante dado a partir de la transformada Fourier de la función de autocorrelación instantánea, a veces referida como la función de covarianza central (Fig. 3). Así, es posible estimar la evolución temporal de la frecuencia y la amplitud de los componentes de señales no estacionarias (Cohen 1995).

\section{Señal no estacionaria}

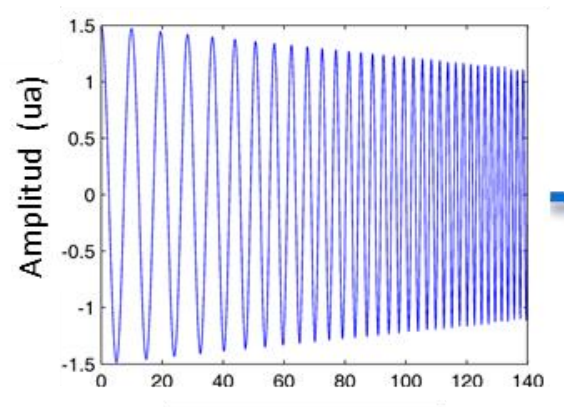

Tiempo (s)

Estimación de potencia y frecuencia
instantáneas

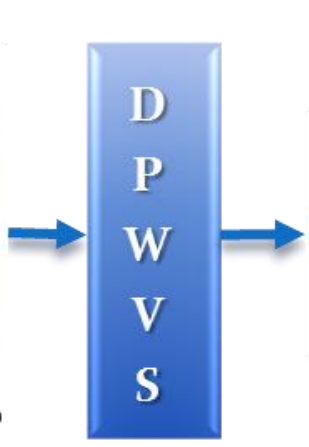

S

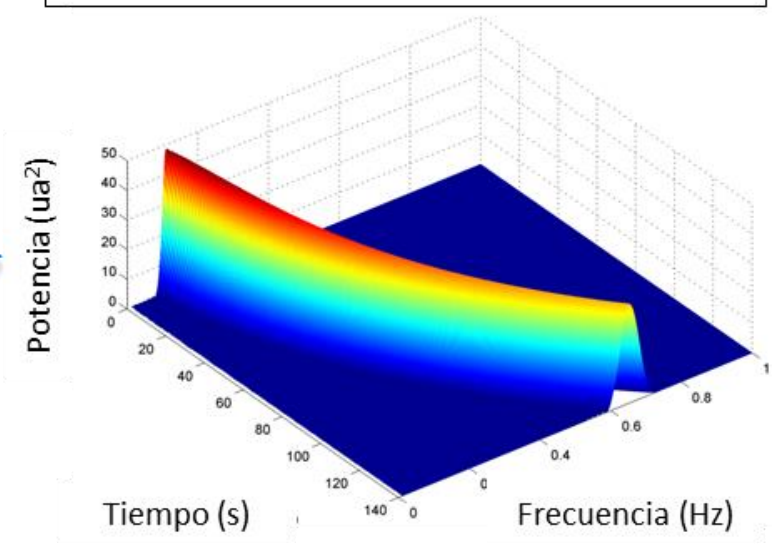

Fig. 3. Las Distribuciones Frecuencia-Tiempo estiman la potencia de los componentes en frecuencia de una señal y cómo cambian a través del tiempo. En la representación en tres dimensiones, la altura refleja la densidad de potencia espectral.

Aunque existen varios tipos de DTF, no hay un criterio único para seleccionar alguna en particular: es necesario considerar las características de la señal a analizar y evaluar qué propiedades deben satisfacerse para obtener información confiable.

En el contexto del análisis espectral de señales fisiológicas, la DTF más ampliamente evaluada y validada (Jasson et al. 1997, Mansier et al. 1998) es la DPWVS, la cual, en su forma discreta, está definida por:

$\operatorname{DPWVS}_{x}^{N, M}(n, \omega)=\sum_{m=-\infty}^{+\infty} g_{M}(m) \sum_{k=-\infty}^{\infty} h_{N}^{2}(k) x(n+k+m) x^{*}(n-k+m) e^{-2 j \omega k}$ donde: 
$D P W V S_{x}(n, \omega)=$ distribución tiempo-frecuencia de la serie de tiempo $\mathrm{x}(\mathrm{n})$

$x(n)=$ serie de tiempo analizada

$g_{M}(m)=$ ventana de suavizado en tiempo

$h_{N}(k)=$ ventana de suavizado en frecuencia

Las propiedades de la DPWVS dependen de las características de las ventanas de suavizado en tiempo y frecuencia, las cuales controlan la resolución tiempo-frecuencia de la distribución. Con respecto a otras representaciones de la clase de Cohen, la DPWVS se caracteriza porque su kernel es separable, por lo que los suavizados en tiempo y en frecuencia puede ajustarse de manera independiente, dotándola de gran flexibilidad en los criterios de suavizado, sus posibles aplicaciones y su eficiencia computacional (Monti et al. 2002). En el caso particular del análisis de la VFC, se requiere una mejor resolución en el tiempo que en la frecuencia, ya que el espectro tiene pocos componentes pero se desea seguir sus cambios de manera cuasi instantánea, cosa que la DPWVS consigue de manera eficiente (Mainardi 2009).

Normalmente, la potencia y frecuencia instantáneas de los componentes espectrales de la VFC se extraen aprovechando las propiedades marginales y del primer momento de la DPWVS, de tal manera que:

$$
\begin{aligned}
& P_{c}(t)=\sum_{m=m_{j}}^{m_{k}} D P W V S\left(f_{m}, t\right) \Delta f \\
& f i_{c}=\frac{\sum_{m=m_{j}}^{m_{k}} f_{m} \cdot \operatorname{DPWVS}\left(f_{m}, t\right)}{\sum_{m=m_{j}}^{m_{k}} \operatorname{DPWVS}\left(f_{m}, t\right)}
\end{aligned}
$$

Donde la banda del componente en cuestión está limitada por $\left[\mathrm{f}_{\mathrm{mj}}, \mathrm{f}_{\mathrm{mk}}\right]$.

\section{Modelos Autorregresivos Variantes en el Tiempo}

$\mathrm{El}$ análisis de una señal mediante MAVT se basa en asumir que dicha señal es generada por un modelo paramétrico:

$$
x(t)=\varphi(t)^{T} \cdot \theta(t)+d(t)
$$

Donde t es el índice de tiempo discreto, $\theta(\mathrm{t})$ es el vector de parámetros variantes en el tiempo, es decir, los coeficientes del modelo, $\phi(t)$ es el vector de observaciones y $d(t)$ es un término de ruido blanco. 
El vector de parámetros es dependiente del tiempo para considerar que la dinámica del sistema puede cambiar en el tiempo. Este vector es desconocido y debe estimarse a partir de los datos, para lo cual se dispone de varios algoritmos. Entre los más utilizados para el análisis de la VFC se encuentran la estimación por mínimos cuadrados recursiva (RLS, por sus siglas en inglés Recursive Least Squares), cuyo objetivo es minimizar el error de predicción del modelo, es decir, la diferencia entre la señal analizada y la aproximación estimada por el modelo (Mainardi et al. 2002). Este algoritmo incorpora un factor de olvido $\lambda$ que debe valer entre 0 y 1 , para controlar la sensibilidad del algoritmo a los cambios, cuya selección es crucial y no trivial, ya que se genera un compromiso entre la capacidad del modelo para seguir cambios rápido y su sensibilidad al ruido: entre más pequeño sea el valor de $\lambda$, el modelo reaccionará mejor a los cambios pero será más sensible al ruido, pero entre más cercano a uno sea, el algoritmo será insensible al ruido pero incapaz de seguir cambios rápidos. Teóricamente, existe un valor óptimo para el factor de olvido para el cual se minimiza el error de estimación de los parámetros, pero éste depende de otros parámetros que tampoco se conocen. Lo recursivo del algoritmo consiste en que, para cada nueva muestra de la señal a analizar, el vector de parámetros es actualizado con una cantidad proporcional al error de predicción del modelo. Entre mayor sea el error, mayor será la variación de los parámetros del modelo. La ganancia del algoritmo, que se actualiza en cada paso, es clave, ya que determina qué tanto impactan los errores de predicción en la actualización de los parámetros. Una versión alternativa de este algoritmo, denominado "olvido ballena" (whale forgetting) emplea una regla de recursión más generalizada con la finalidad de reducir el dramatismo del compromiso entre sensibilidad al cambio y sensibilidad al ruido, generando una función de ponderación más suave que considera el promedio de los datos más recientes, por lo que, aunque preserva la reactividad a los cambios en la estimación de los parámetros es menos sensible al ruido (Bianchi et al. 1997).

Para la estimación de las potencias de los componentes de BF y AF se utiliza un método de descomposición espectral, que consiste en dividir el área bajo el espectro derivado del modelo MAVT en una suma de componentes cuyas frecuencias centrales y potencias pueden ser calculadas de manera relativamente sencilla, pero que implica la detección continua de los polos MAVT. Esta tarea ardua de por sí y consume mucho tiempo, pero se complica aún más cuando los parámetros del modelo se actualizan constantemente y se requiere que para estimar los nuevos polos se consideren los valores de los polos estimados anteriormente, por lo que se han propuesto 
algoritmos de seguimiento de polos (Meste et al. 2005), aunque ligeras variaciones en los polos del modelo pueden ocasionar grandes variaciones en la potencia espectral estimada. Finalmente, para calcular la potencia de los componentes $\mathrm{BF}_{\mathrm{RR}} \mathrm{y} \mathrm{AF}_{\mathrm{RR}}$ se suman las potencias de todos los componentes calculados cuyas frecuencias centrales se encuentren en las bandas elegidas.

\section{Demodulación Compleja}

En cuanto a la DC, se presupone que la señal a analizar $x(\cdot)$ tiene un componente de interés en la banda $\left[\mathrm{f}_{0}-\mathrm{f}_{\omega}, \mathrm{f}_{0}+\mathrm{f}_{\omega}\right]$. La señal es entonces demodulada al multiplicarla por $e^{j-2 \pi f_{0}}$ para recorrer el espectro y centrar la banda de interés en el eje $f=0$. El siguiente paso consiste en aplicar un filtro paso bajo con frecuencia de corte $\mathrm{f}_{\omega}$, obteniéndose así el componente deseado $x_{f_{0}}(t)$. Si los componentes de la señal están restringidos a una banda específica, el valor de $\mathrm{f}_{\omega}$ seleccionado puede ser lo suficientemente pequeño como para que $x_{f_{0}}(t) \approx|a(t)| e^{j \theta(t)}$ y la potencia $\mathrm{y}$ frecuencia instantáneas del componente pueden ser estimados como:

$$
\begin{gathered}
P_{x_{f o}}=|a(t)|^{2} \\
f i_{x_{f 0}}=\frac{1}{2 \pi\left(\frac{d \theta}{d t}\right)}
\end{gathered}
$$

Este procedimiento debe repetirse para todas las frecuencias de interés (Hayano et al. 1993). La principal limitación de la $\mathrm{DC}$ es la selección de las frecuencias centrales $\mathrm{f}_{0} \mathrm{y}$ las frecuencias de corte $\mathrm{f}_{\omega}$, que no es trivial como podría aparentar, particularmente cuando las frecuencias centrales de los componentes varían continuamente sobre una banda amplia.

\section{Selección del método idóneo}

Puesto que no hay un método de referencia para calcular unívocamente el "espectro instantáneo" de una señal no estacionaria y por lo tanto no se puede comparar el desempeño de los diferentes algoritmos en el análisis de señales reales, para el análisis de la VFC se consideran relevantes algunos requerimientos de los algoritmos relacionados con su capacidad de identificar y cuantificar los cambios de los componentes espectrales, sus posibilidades de ajustar la resolución entiempo y frecuencia de manera independiente y de obtener mediciones cuantitativas de las dinámicas de los componentes espectrales a partir de las representaciones tiempo-frecuencia (Mainardi et al. 2002).

Aunque los MAVT permiten el ajuste independiente de las resoluciones en tiempo (por el factor de olvido) y en frecuencia (por el orden del modelo), proveen un espectro suavizado con picos 
bien definidos que permiten identificar y rastrear fácilmente sus frecuencias centrales y la cuantificación de los parámetros espectrales puede obtenerse mediante descomposición espectral automática, presentan limitaciones importantes. Éstas se relacionan con su extrema dependencia de una apropiada selección del factor de olvido y el orden del modelo, ya que una selección inadecuada puede hacer que los resultados sean muy lejanos a la realidad, en particular cuando la señal es no estacionaria y presenta transitorios abruptos, ya que puede volverse altamente inestable. Más aún, la estimación cuantitativa de las potencias y frecuencias instantáneas de los componentes espectrales es altamente sensible a la ubicación y tipo de polos del modelo (Mainardi 2009). Para resolver estos problemas se han propuesto y aplicado diversas mejoras al algoritmo RLS, que incluyen factores de olvido variantes en el tiempo, órdenes del modelo actualizables y funciones de olvido generalizadas como el whale forgetting (Bianchi et al. 1997) y algoritmos de seguimiento de polos más robustos (Meste et al. 2005). Sin embargo, las mejoras en desempeño se obtienen a costa de mayor complejidad computacional.

Las ventajas principales de la DPWVS son su eficiencia computacional y excelentes resoluciones tiempo-frecuencia, que también pueden ser ajustadas de manera independiente. La interferencia por términos cruzados es el principal problema de esta metodología, pero una apropiada elección de las ventanas de suavizado en tiempo y frecuencia permiten minimizarlos e incluso estimar la coherencia tiempo-frecuencia entre dos señales (Orini et al. 2012).

En cuanto a la demodulación compleja, su facilidad de implementación y eficiencia computacional resultan sumamente atractivas, pero su principal limitación es la selección de las frecuencias central y de corte del filtro de demodulación, la cual se puede facilitar si se incluye información a priori sobre las frecuencias centrales de los componentes de interés, que puede obtenerse por otro método de análisis como la DPWVS (Monti et al. 2002) o del procesamiento de otra señal como la respiración (Bailón et al. 2006).

\section{Arritmia sinusal respiratoria}

La VFC es modulada por la respiración. Este fenómeno es conocido como arritmia sinusal respiratoria (ASR). Al comparar los registros simultáneos de frecuencia cardiaca y respiración en el tiempo podemos apreciar que durante la inspiración la frecuencia cardiaca aumenta, mientras en la espiración disminuye (Fig. 4A). En el dominio de la frecuencia, la ASR se manifiesta como la coherencia que existe entre los espectros de potencia de la señal respiratoria y del tacograma, en 
particular del componente de $\mathrm{AF}_{\mathrm{RR}}$ (Fig. 4B). La existencia y amplitud de la ASR se consideran herramientas valiosas para caracterizar y evaluar el estado de salud de la rama parasimpática del control autonómico cardiovascular, en particular, se le considera un indicador del tono vagal (Yasuma y Hayano 2004, Hrushensky et al. 1984).

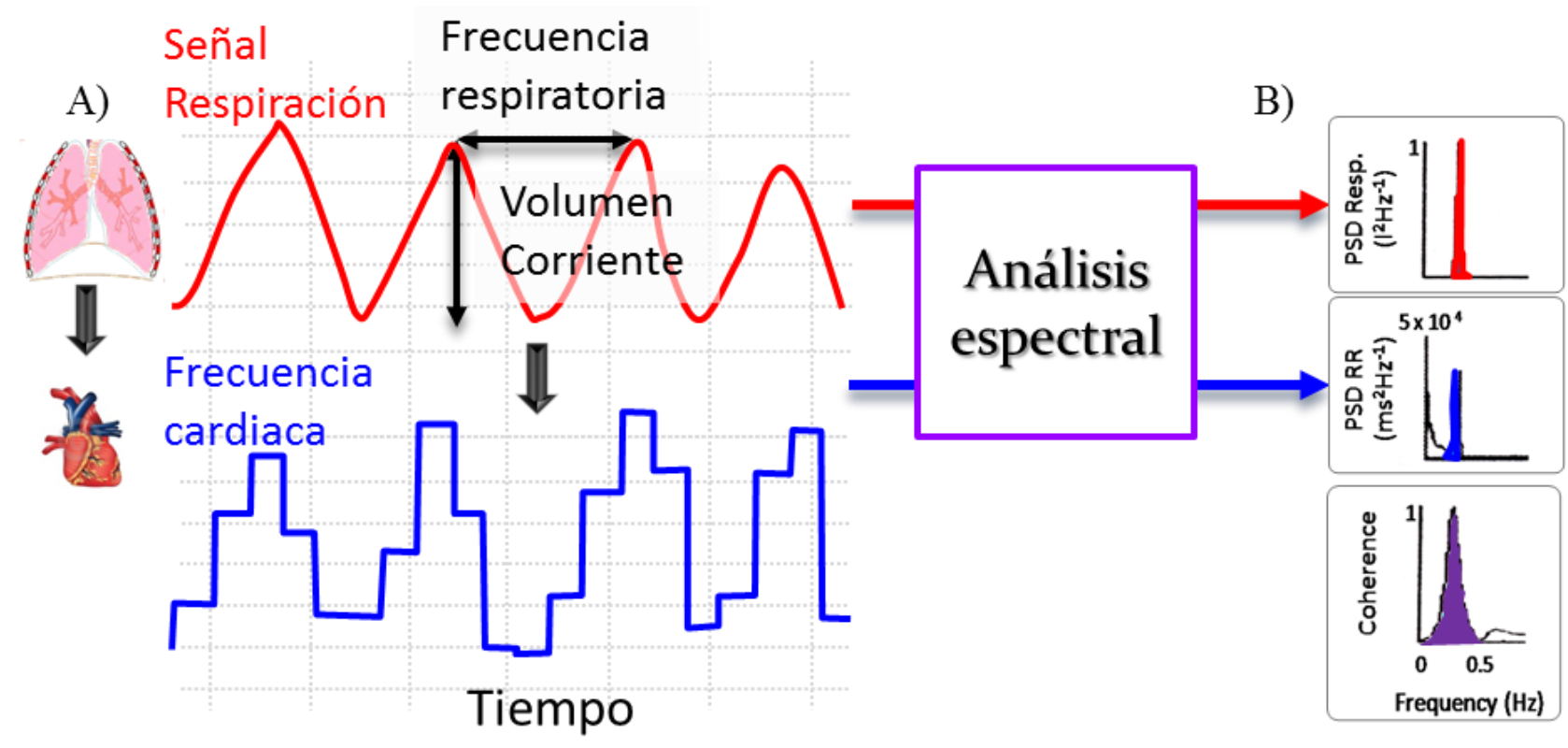

Fig. 4. El fenómeno fisiológico de la arritmia sinusal respiratoria (ASR). A) En el tiempo: acoplamiento entre la frecuencia cardiaca y los movimientos respiratorios. B) En la frecuencia: coherencia entre el componente de alta frecuencia de los intervalos RR y el componente de la respiración.

\section{Fisiología de la ASR}

Aunque los mecanismos fisiológicos que la originan y su posible función evolutiva aún son temas controversiales (Hayano y Yasuma 2003, Eckberg 2009, Karemaker 2009, Tzeng et al. 2009), actualmente se considera que en la generación de la ASR participan dos mecanismos, uno central y uno periférico, integrados por los núcleos vagales del tallo cerebral que inervan al nodo sinusal (Fig. 5). El mecanismo central está constituido por la influencia directa de los núcleos de control respiratorio del tallo cerebral sobre los núcleos vagales. El mecanismo periférico está dado por la influencia combinada de los barorreceptores, quimiorreceptores y aferencias pulmonares (Saul y Cohen 1994).

Durante la inspiración, ocurre inhibición de los núcleos vagales y aumento de la actividad simpática, por lo que la frecuencia cardiaca aumenta y se reduce la potencia de $\mathrm{AF}_{\mathrm{RR}}$. Durante la espiración, aumenta la actividad de los núcleos vagales reduciendo la frecuencia cardiaca y aumentando la potencia de $\mathrm{AF}_{\mathrm{RR}}$. Este fenómeno es conocido como compuerta respiratoria (Fig. 
5), la cual se cierra durante la inspiración y se abre con la espiración (Gilbey et al. 1984). La frecuencia de trabajo de la compuerta corresponde a la FR. Así, cuando la FR es baja, la amplitud de la ASR es mayor, y cuando la FR se incrementa, la amplitud de la ASR disminuye (Eckberg 2003).

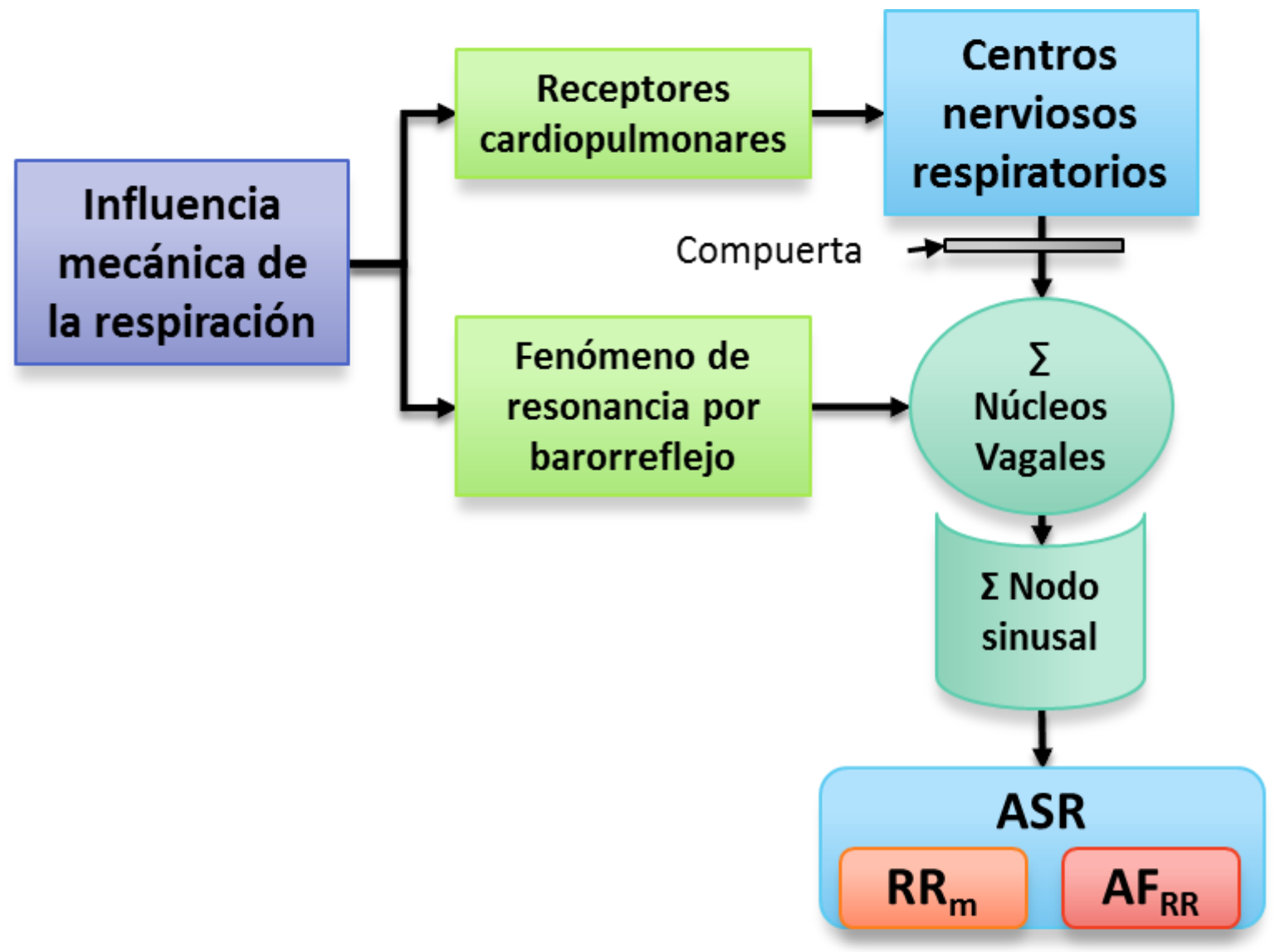

Fig. 5. Mecanismos fisiológicos aceptados como generadores de la arritmia sinusal respiratoria (ASR) y sus indicadores temporal y espectral, el valor medio de los intervalos $R R\left(R R_{m}\right)$ y la potencia de su componente de alta frecuencia $\left(\mathrm{AF}_{\mathrm{RR}}\right)$. Actualmente, se acepta que la respiración ejerce su influencia sobre el control autonómico de la función cardiovascular a través de mecanismos centrales, mediados por la interacción de los centros nerviosos respiratorios y de los núcleos vagales, y periféricos, dependientes del barorreflejo.

\section{Factores que modifican la amplitud de la ASR}

Un estudio clásico (Hirsch y Bishop 1981) estableció que la amplitud de la ASR depende principalmente de las dos variables ventilatorias: la FR y el volumen corriente (VC). Según estos autores, la relación entre la FR y la amplitud de la ASR muestra un comportamiento similar al de un filtro pasa-bajas (Fig. 6A). El aumento del VC tiene el efecto de aumentar la ganancia del filtro, es decir, que a mayor VC le corresponderá un mayor valor de ASR (Fig. 6B). A pesar de 
que el efecto del VC se obtuvo con pocos puntos y la relación ASR-VC no fue explícitamente reportada ni analizada, estos resultados han tenido una profunda influencia en el estudio de la ASR: nadie cuestiona que su magnitud sea directamente proporcional a la del VC.
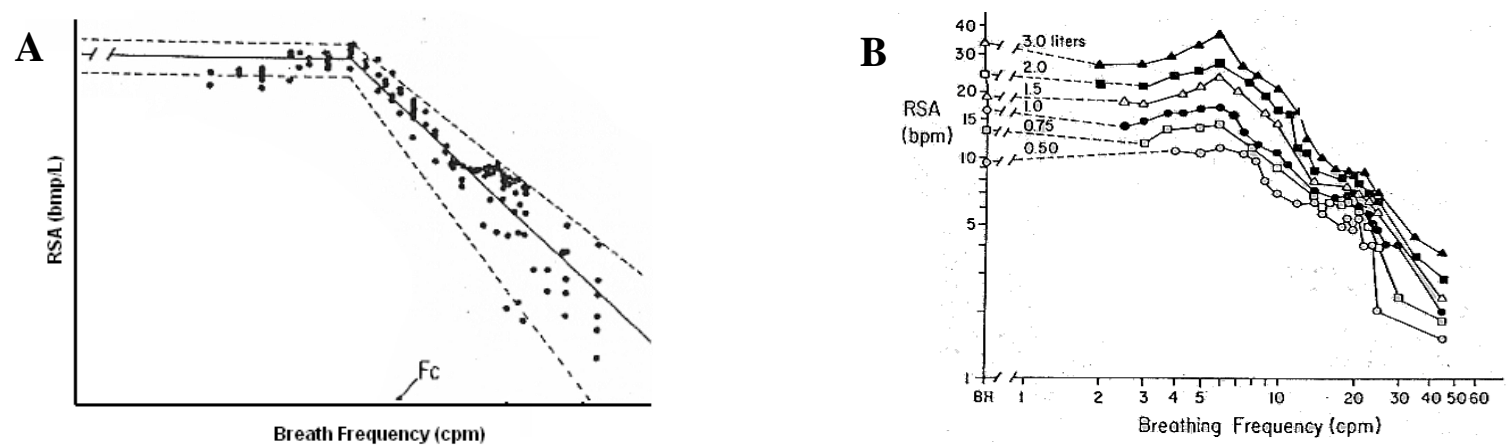

Fig. 6. A) Comportamiento como filtro pasa bajas de la amplitud de la arritmia sinusal respiratoria: a mayor frecuencia respiratoria, menor amplitud. B) Efecto del volumen corriente: a mayor volumen, mayor amplitud. Tomado de Hirsch y Bishop (1981).

Otro factor que parece relacionarse con la amplitud de la ASR es el nivel de $\mathrm{CO}_{2}$ en la sangre. La ventilación pulmonar (VP) o volumen minuto, resultado del producto VC x FR, es uno de los principales factores que determinan el nivel de $\mathrm{CO}_{2}$ en la sangre: la hiperventilación causa hipocapnia y la hipoventilación resulta en hipercapnia. Sasano et al. (2002) reportaron que al incrementar la presión alveolar de $\mathrm{CO}_{2}\left(\mathrm{PCO}_{2}\right)$ de 30 a 40 y $50 \mathrm{mmHg}$ la amplitud de la ASR aumentó proporcionalmente (Fig. 7), efecto que estaría mediado por el quimiorreflejo sobre los núcleos de control autonómico. Concluyeron que los cambios observados en la ASR podrían facilitar la relación ventilación - perfusión (Hayano et al. 1996, Sasano et al. 2002). 

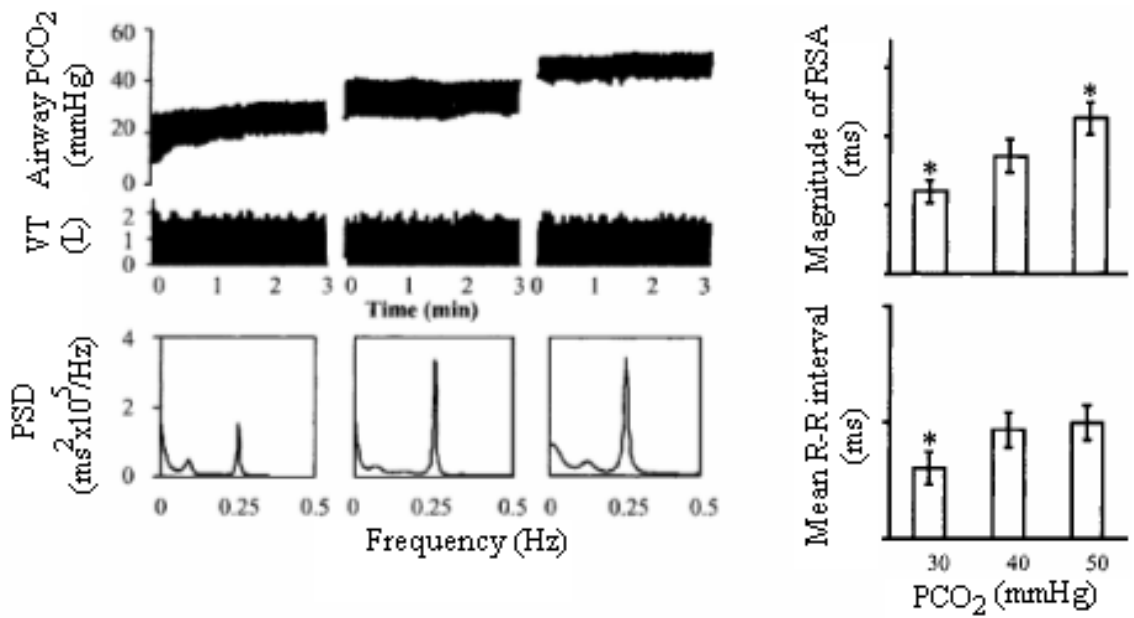

Fig. 7. Relación entre los aumentos de la presión alveolar de $\mathrm{CO}_{2}\left(\mathrm{PCO}_{2}\right)$ y la amplitud de la ASR: conforme se incrementa la $\mathrm{PCO}_{2}$, aumenta también la potencia de $\mathrm{AF}_{\mathrm{RR}}$ y el $\mathrm{RR}_{\mathrm{m}}$. Tomado de Sasano et al. (2002).

\section{Metodologías para la evaluación de la ASR}

Puesto que no existe una metodología estándar para evaluar el fenómeno de la ASR (Grossman y Taylor 2007), se han desarrollado numerosos protocolos que difieren en diversos aspectos, como la duración de la maniobra, el rango y modo de variación de la FR, si controlan o no el VC y el tipo de análisis utilizado (Hirsch y Bishop 1981, Saul et al. 1989, Taylor et al. 2001). La mayoría incluye la variación de la FR en etapas de varios minutos de duración, necesarios para que la frecuencia cardiaca alcance lo que los autores consideran un estado estable. Estos protocolos son largos y cansados, llegando a producir hipocapnia en los sujetos de investigación por sostener durante periodos extensos una respiración voluntariamente controlada, es decir, no regida por el quimiorreflejo. La larga duración y la dificultad de ejecución de estos protocolos imposibilitan el estudio de los efectos de maniobras vagales o simpáticas sobre la relación $A_{F_{R R}}-\mathrm{FR}$ completa. Más aún, se han publicado pocos trabajos en los cuales evaluaron el efecto del VC sobre la amplitud de la ASR (Kollai et al. 1990, Kobayashi et al. 1998).

El control voluntario de los movimientos respiratorios es un procedimiento muy valioso que ha permitido explorar los efectos de la FR y el VC sobre la ASR. Este conocimiento es necesario para comprender los mecanismos subyacentes a este fenómeno (Hirsch y Bishop 1981, Larsen et al. 2010). Una de las herramientas más poderosas y utilizadas para la evaluación de la ASR es el análisis espectral de la VFC, en particular la potencia de $\mathrm{AF}_{\mathrm{RR}}$ (Larsen et al. 2010), cuyos cambios en amplitud y frecuencia central son fácilmente medibles. El análisis espectral también permite la estimación de la coherencia entre las series de tiempo de los intervalos RR y la respiración. 


\section{Efectos del cambio de postura sobre la actividad autonómica}

Durante el cambio de postura activo (CPA) la gravedad causa el desplazamiento del volumen sanguíneo hacia los miembros inferiores, ocasionando un descenso en la presión arterial. Para contrarrestar la hipotensión, los barorreceptores envían señales hacia los centros autonómicos, produciendo un incremento en la salida simpática cardiaca y vasomotora y una disminución o retiro de la actividad vagal. Estos cambios causan a su vez aumentos de la resistencia periférica, de la FC y de la contractilidad cardiaca, por lo tanto del gasto cardiaco y en consecuencia de la presión arterial media. El efecto del CPA sobre la potencia espectral de la VFC fue establecido por Pomeranz et al. (1985): el componente de $\mathrm{BF}_{\mathrm{RR}}$ aumentó y el de $\mathrm{AF}_{\mathrm{RR}}$ disminuyó (Fig. 8).

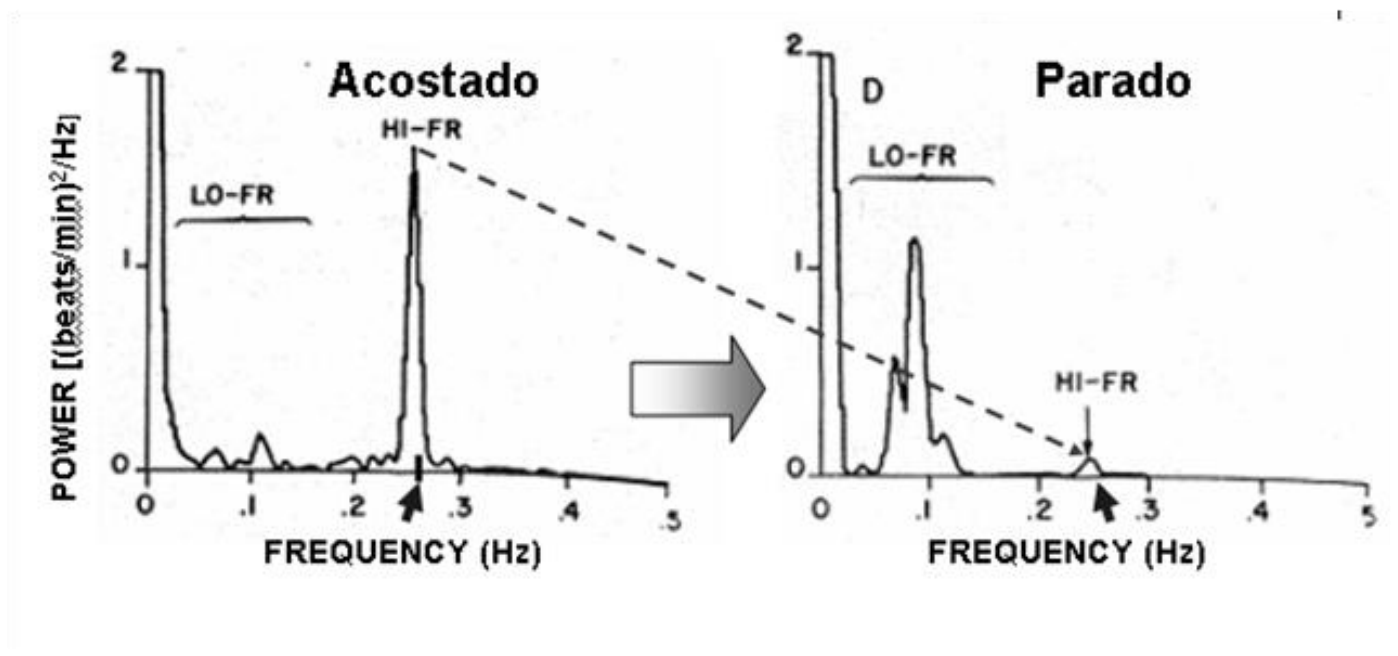

Fig. 8. Efecto del cambio de postura sobre los componentes espectrales de la variabilidad de la frecuencia cardiaca: al erguirse, la potencia del componente de alta frecuencia disminuye y la del de baja frecuencia aumenta. Tomado de Pomeranz et al. (1985).

\section{Diferencias entre sexos en el control autonómico-cardiovascular}

Estudios previos que compararon entre los valores basales de ASR de hombres y mujeres o sus cambios en respuesta a maniobras específicas han rendido resultados controversiales. En un estudio en gemelos, las mujeres presentaron mayor ASR que los hombres en condición de reposo (Snieder et al. 2007). En respuesta a estimulación con frío en la frente, los hombres mostraron mayores incrementos en ASR y más reducciones en frecuencia cardiaca que las mujeres (Ruiz et al. 2006). La respuesta de la ASR a un VC del doble del valor de reposo con FR espontánea fue similar en ambos sexos (Sébert 1983). Además, ni el periodo cardiaco ni la potencia de $\mathrm{AF}_{\mathrm{RR}}$ en unidades absolutas fueron diferentes entre sexos sin importar las condiciones respiratorias (Stark et al. 2000). 


\section{Enfoque novedoso para el estudio de la integración autonómica}

La integración de múltiples aferencias es intrínseca al control autonómico cardiovascular, razón por la cual en ocasiones es difícil establecer a qué aferencia y en qué grado se debe un cambio en la salida o respuesta estudiada (Fig. 9).

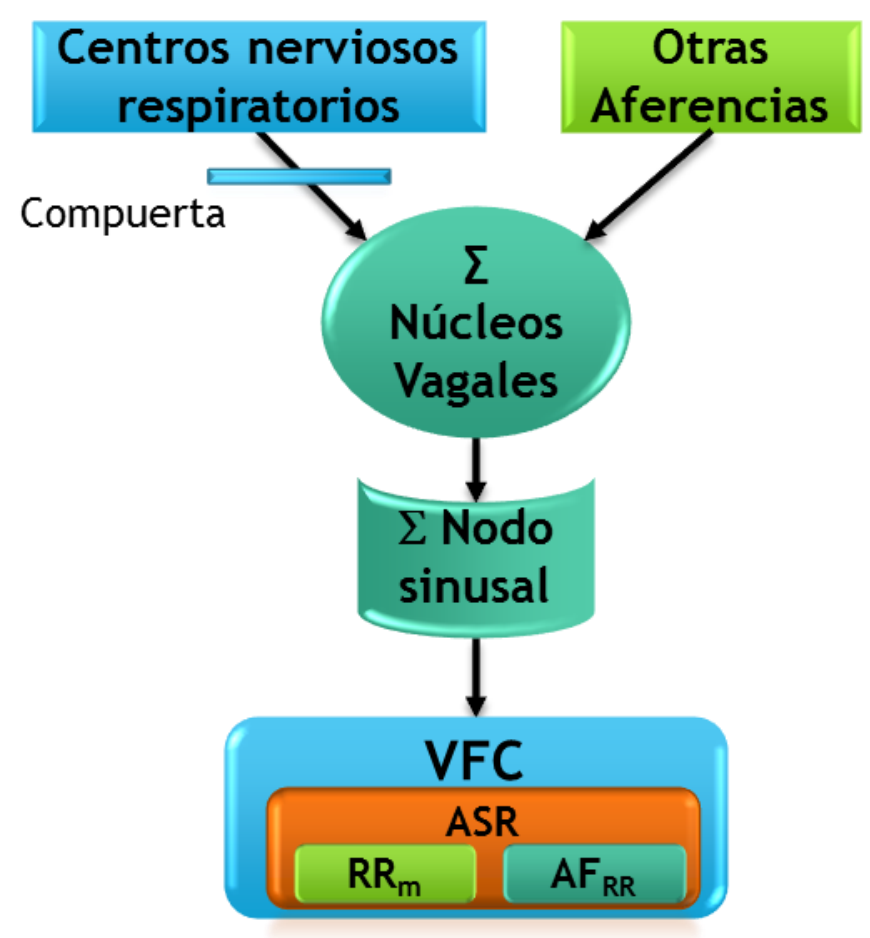

Fig. 9. Las eferencias de los núcleos autonómicos parasimpáticos resultan de la integración de diversas influencias aferentes, por lo que la interpretación de sus indicadores no invasivos, derivados del análisis en el tiempo y la frecuencia de la variabilidad de la frecuencia cardiaca (VFC) es ambigua.

En este proyecto propone el empleo de un enfoque novedoso para estudiar este fenómeno, el cual consiste en analizar las respuestas cardiovasculares a la aplicación independiente de dos estímulos de efecto conocido, y compararlas con la respuesta a la aplicación de los mismos estímulos de manera simultánea. La comparación entre los efectos provocados en la actividad autonómica por la variación independiente y simultánea de la FR y el VC podría indicar si la integración de las aferencias respiratorias realizada por los núcleos autonómicos es potenciadora, aditiva, oclusiva o inhibidora, de manera análoga a estudios que comparan entre los efectos corticales de estimulación somatosensorial independiente y simultánea para explorar los grados de sobrelapamiento de las áreas responsivas (Biermann et al. 1998, Hamada y Suzuki 2003). En estos 
estudios se emplea el llamado Índice de Interferencia (II) para determinar el tipo de interacción entre estímulos, el cual se calcula de la siguiente manera:

$$
I I=\frac{\text { Respuesta }(E 1+E 2)}{\text { RespuestaE } 1+\text { RespuestaE2 }}
$$

Si el valor del II es menor que 1, indica que los estímulos E1 y E2 se ocluyen entre sí, es decir, la respuesta ante la aplicación simultánea de los estímulos es menor que la suma de las respuestas producidas por cada estímulo de manera independiente. Si el II es igual a 1, la interacción entre E1 y E2 es aditiva, es decir, la respuesta del sistema al estímulo simultáneo es igual a la suma de la respuesta a cada estímulo por separado. Finalmente, si el II es mayor que 1, la respuesta al estímulo simultáneo es mayor que la suma de las respuestas a los estímulos por separado, lo que indica que los estímulos se potencian entre sí.

En particular, se propone emplear estímulos que varíen lineal y continuamente en el tiempo, de tal manera que, al analizar la respuesta del sistema estudiado, sea posible determinar qué tanto se aleja de la linealidad (Fig. 10). En el caso más deseable, la respuesta obtenida también será lineal, lo que facilitaría su caracterización formal al reducirla a dos parámetros, pendiente e intercepto. Los cambios en la respuesta podrían ser evaluados por la comparación de únicamente dos valores que representarían a toda una función, en contraposición con la fisiología de puntos discretos y normalmente insuficientes para hacer una interpolación satisfactoria.

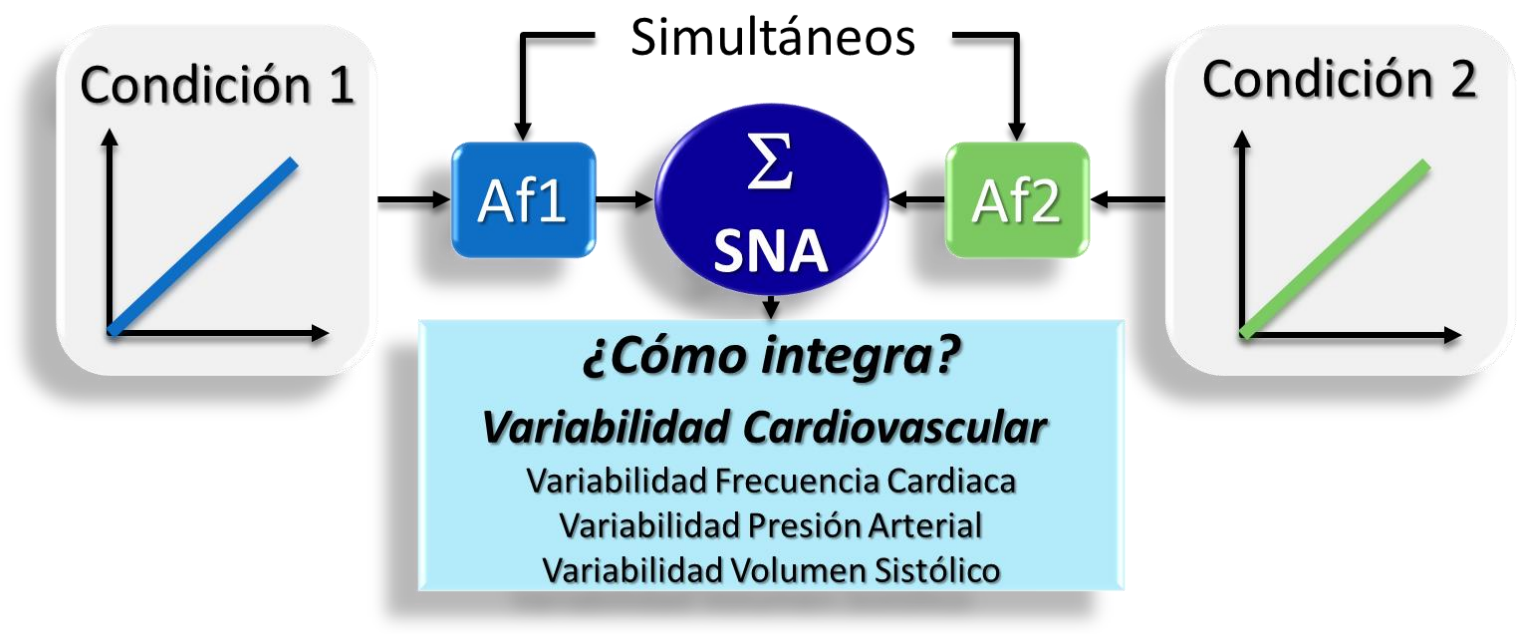

Fig. 10. Enfoque novedoso para el estudio de la integración autonómica de varias aferencias, consistente en la comparación de la respuesta cardiovascular a la aplicación individual de estímulos lineal y continuamente variantes en el tiempo con la respuesta a la aplicación simultánea de los mismos estímulos. 


\section{Justificación}

Actualmente, el análisis de la VFC puede considerarse la mejor herramienta disponible para el estudio no invasivo de la función y control autonómico cardiovascular en seres humanos (Task Force, Parati et al. 2006). Sin embargo, una búsqueda en PubMed (empleando los términos "heart rate variability" AND "spectral analysis" y filtrando los resultados a estudios en seres humanos publicados en los últimos 10 años), revela que la mayoría (75\%) de los estudios publicados que la emplean tienen

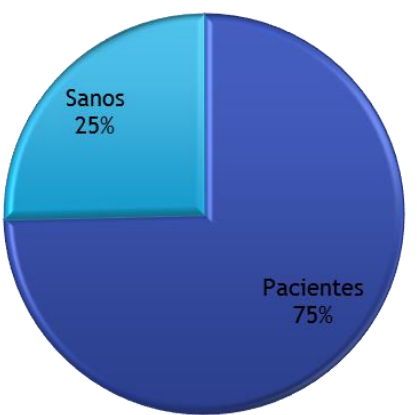

Fig. 11. Representación gráfica de la proporción de estudios que emplean el análisis de la VFC con un enfoque clínico y los que tienen un enfoque fisiológico.

un enfoque clínico, es decir, que sus sujetos de estudio padecen alguna enfermedad o daño funcional; pocos se dedican a estudiar el funcionamiento normal en el ser humano sano (Fig. 11).

La potencia del componente de $\mathrm{AF}_{\mathrm{RR}}$ es el índice no invasivo de la actividad vagal más validado (Parati et al. 2006). Desafortunadamente, generalmente los investigadores que lo emplean pasan por alto la estrecha relación de este componente con la respiración (Brown et al. 1993). De los artículos publicados sobre el análisis espectral de la VFC en seres humanos los últimos 10 años (489 en PubMed), la mayoría no registró o reportó la respiración (403, equivalente al 82.4\%); de los pocos que la reportaron 94\% no la controlaron, y los que sí la controlaron emplearon un solo valor de FR arbitrariamente seleccionado. Más aún, casi ninguno (1 de 86) controló el VC (Fig.

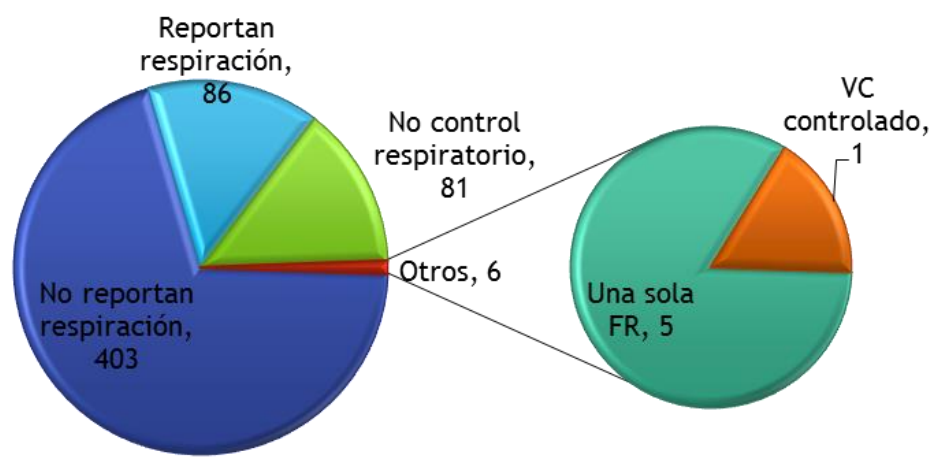

Fig. 12. A pesar de que la estrecha relación entre la potencia del componente de $A F_{R R}$ y la respiración es ampliamente reconocida y aceptada, al no registrar y menos aún controlar la FR y/o el VC, el posible origen de los cambios de este indicador de actividad vagal resulta ambiguo.
12). Esta situación deja entrever el poco conocimiento y atención que prevalecen sobre la compleja interacción entre los núcleos de control respiratorio y los núcleos vagales (Eckberg 2003): si no se conocen las variaciones que pueden ocurrir en la respiración, la interpretación de los cambios del componente $\quad \mathrm{AF}_{\mathrm{RR}} \quad$ resultará ambigua. 
Actualmente, no existe un protocolo de control respiratorio estandarizado para el estudio de la ASR. La mayoría de los protocolos empleados duran decenas de minutos, son difíciles de realizar y producen fatiga en los sujetos de experimentación. Además, las variaciones entre los protocolos dificultan la comparación de los resultados. Así, el desarrollo de una metodología estandarizada para la evaluación de la ASR resulta una meta necesaria.

A pesar de que la ASR ha sido estudiada desde hace décadas, los efectos del VC y la FR sobre su amplitud están insuficientemente formalizados, ya que aún no se cuenta con ecuaciones que relacionen estas tres variables simultáneamente. Hirsch y Bishop (1981) publicaron uno de los estudios más influyentes que documentaron los efectos de la FR y el VC en la magnitud de la ASR. En este estudio, emplearon dos protocolos de control respiratorio diferentes, uno para examinar los efectos de más de 10 diferentes FR a VC fijo, y otro para evaluar los efectos de seis diferentes VC a FR constante. Reportaron que la amplitud de la ASR presenta una relación inversa con la FR y una relación lineal positiva con el VC. Esta última relación es asumida como cierta para normalizar la amplitud de la ASR respecto al VC, procedimiento considerado necesario para eliminar la interferencia respiratoria, minimizar las variaciones interindividuales y mejorar su comparabilidad (Kobayashi 1998, Grossman y Taylor 2007, Larsen et al. 2010). Los hallazgos de ese estudio sobre las influencias independientes de cada variable respiratoria han sido confirmados repetidamente (Grossman y Taylor 2007), más frecuentemente para la FR que para el VC. Sin embargo, no hay reportes disponibles que documenten los efectos de la variación continua y simultánea de las dos variables respiratorias sobre la amplitud de la ASR, a pesar de que las variaciones espontáneas en la respiración generalmente involucran cambios simultáneos en ambas variables.

Aunque la FR es la variable modificadora de la ASR que ha sido más estudiada, sus efectos aún no están completamente delineados, debido a que la mayoría de los trabajos emplea una sola (Badra et al. 2001, Pinna et al. 2006) o unas pocas frecuencias respiratorias discretas seleccionadas arbitrariamente (Cooke et al. 1998) y que se ubican en un rango estrecho de valores (Fig. 13). Así, se han empleado pocos puntos para describir las relaciones, y queda por explorar si existen valores límite en FR más allá de los cuales la ASR desaparece. 
ASR

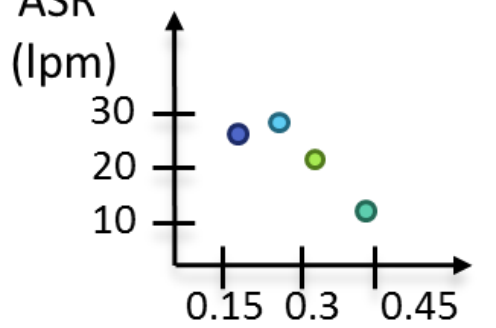

$\mathrm{FR}(\mathrm{Hz})$

Rango estrecho, pocos puntos, valores arbitrarios
ASR

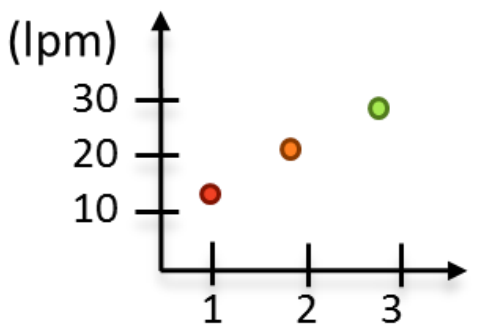

Rango estrecho, pocos puntos, pocos estudios

Fig. 13. Los protocolos generados para el estudio del efecto de las variables respiratorias sobre la amplitud de la ASR emplean pocos valores arbitrariamente seleccionados que caen en un rango estrecho y son aplicados de manera discontinua, por lo que se obtiene poca información que generalmente se reporta como valores medios.

El efecto de la amplitud del VC sobre la ASR ha sido todavía menos estudiado: en los estudios disponibles o no se controla el VC (Tzeng et al. 2009), o se mantiene un VC constante arbitrario a diferentes frecuencias (Cooper et al. 2004), o se emplean varios niveles arbitrarios de VC sostenidos varios minutos para tratar de conocer su efecto sobre la ASR (Kobayashi 1998). De nueva cuenta, aún no se formaliza el efecto del VC sobre la ASR, y se desconoce si existen valores umbrales mínimos y/o máximos (Fig. 13).

Se considera entonces que la formalización de las relaciones continuas entre la FR, el VC y la potencia espectral del componente $\mathrm{AF}_{\mathrm{RR}}$ permitirá contar con un indicador cuantitativo de la actividad vagal que integre el efecto combinado de las variables respiratorias, de tal manera que su variación refleje únicamente la influencia de alguna condición sobrepuesta sobre la actividad autonómica (Fig. 14). Hasta donde sabemos no se han publicado trabajos que empleen un enfoque similar al propuesto en este proyecto para el estudio de la ASR.

Pocos estudios han investigado las diferencias entre sexos en los niveles basales de ASR (Snieder et al. 2007, Mortola et al. 2015), en su respuesta a cambios en las variables respiratorias (Sébert 1983, Stark et al. 2000, Mortola et al. 2015) o a otras maniobras fisiológicas (Sébert 1983, Stark et al. 2000). Por lo tanto, este problema permanece irresuelto.

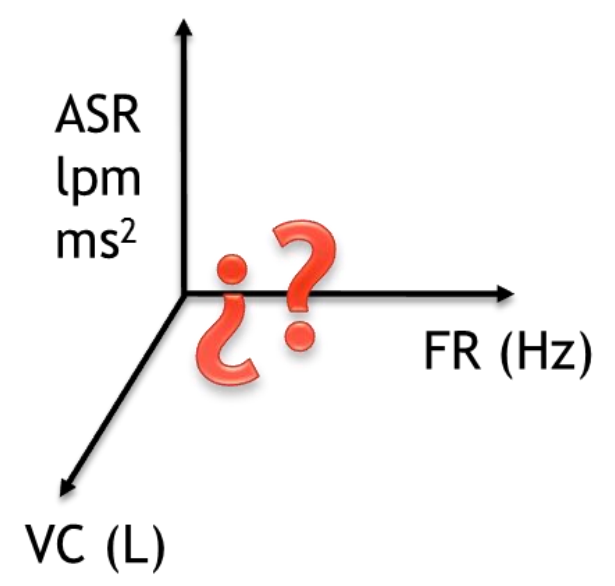

Fig. 14. Hasta la fecha no se ha formalizado el efecto que tiene la variación simultánea y continua de las dos principales variables respiratorias sobre la amplitud de la ASR. 


\section{Hipótesis}

Con base en los siguientes hechos conocidos y aceptados:

1. La ASR es un fenómeno de acoplamiento cardiorrespiratorio de origen central y periférico caracterizado por un incremento de la actividad vagal en la fase espiratoria (Eckberg 2003).

2. El componente $\mathrm{AF}_{\mathrm{RR}}$ de la VFC es el mejor indicador disponible para la evaluación no invasiva de la actividad vagal (Parati et al. 2006).

3. La potencia de $\mathrm{AF}_{\mathrm{RR}}$ varía de manera análoga a un filtro pasa bajas respecto de la FR (Hirsch y Bishop 1981, Saul et al. 1989).

4. La amplitud de la ASR es directamente proporcional al VC (Kobayashi 1998).

5. La hipercapnia aumenta la amplitud de la ASR (Hayano et al. 1996, Yasuma y Hayano 2004), pero una hipocapnia leve no la afecta significativamente (Cooper et al. 2004).

6. Cambiar de una postura supina a una erguida suscita un retiro de la actividad vagal y un incremento en la afluencia simpática, indicadas por una disminución de la potencia de $\mathrm{AF}_{\mathrm{RR}}$ (Pomeranz 1985).

7. Las distribuciones tiempo-frecuencia son herramientas adecuadas para realizar el análisis espectral de señales no estacionarias (Monti et al. 2002, Mainardi 2009).

8. Cuando la FR es mayor que la mitad de la frecuencia cardiaca, la ASR ocurre en rangos de frecuencia menores a aquéllos de la FR (Witte et al. 1988).

9. La amplitud de la ASR en condiciones basales es similar entre géneros (Mortola et al. 2015).

Planteamos las siguientes hipótesis:

1. Con relación a los efectos causados por la variación independiente del VC a FR constante $\left(\mathrm{E}_{\mathrm{VC}}\right)$ y de la FR a VC constante $\left(\mathrm{E}_{\mathrm{FR}}\right)$ sobre la potencia del componente de $\mathrm{AF}_{\mathrm{RR}}$, el efecto de la variación simultánea de FR y VC ( $\left.\mathrm{E}_{\mathrm{VC}+\mathrm{FR}}\right)$ será similar a la suma de sus efectos independientes: $E_{V C+F R} \sim E_{V C}+E_{F R}$, por lo que su II será igual o muy cercano a 1. Si los resultados de la investigación respaldan esta hipótesis, podrían indicar que las regiones del SNA que participan en la generación de la ASR integran por separado las aferencias del VC y la FR.

2. El componente de $\mathrm{AF}_{\mathrm{RR}}$ presentará una función lineal directa con el $\mathrm{VC}$.

3. La relación entre el componente de $\mathrm{AF}_{\mathrm{RR}}-\mathrm{VC}-\mathrm{FR}$ en rangos seleccionados ad hoc será lineal e indicará el retiro vagal provocado por el cambio de postura con una reducción significativa de la potencia de $\mathrm{AF}_{\mathrm{RR}}$. Los coeficientes de las variables independientes de la regresión múltiple 
$A F_{R R}=b_{o}+b_{1} F R+b_{2} V C$ serán menores $(\mathrm{p}<0.05)$ en la condición de parado con respecto a sentado.

4. La relación entre la frecuencia instantánea del componente de $\mathrm{AF}_{\mathrm{RR}}\left(\mathrm{FIAF}_{\mathrm{RR}}\right)$ y la FR permitirá evaluar los límites fisiológicos de la ASR.

5. Los cambios de fracción alveolar de $\mathrm{CO}_{2}$ durante las maniobras no tendrán efectos significativos sobre la amplitud de la ASR.

6. No habrá diferencias entre las respuestas de hombres y mujeres. 


\section{Objetivos}

\section{General}

Generar conocimiento original y relevante sobre la interrelación funcional de la respiración controlada y el control autonómico cardiovascular, con énfasis en la amplitud de la ASR, mediante el análisis de las relaciones entre variables respiratorias e indicadores temporales y espectrales de la actividad vagal, generadas a partir de protocolos de variación continua y lineal de la FR y el VC, aplicados de manera independiente y simultánea.

\section{Específicos}

1. Explorar la manera en que el sistema formado por núcleos vagales-nodo SA responde a la aplicación de dos aferencias simultáneas variantes en el tiempo, la FR y el VC, mediante el análisis cuantitativo de las relaciones continuas que se establecen entre las variables controladas y un valioso indicador no invasivo de la actividad vagal, la potencia del componente de $\mathrm{AF}_{\mathrm{RR}}$ (Fig.15).

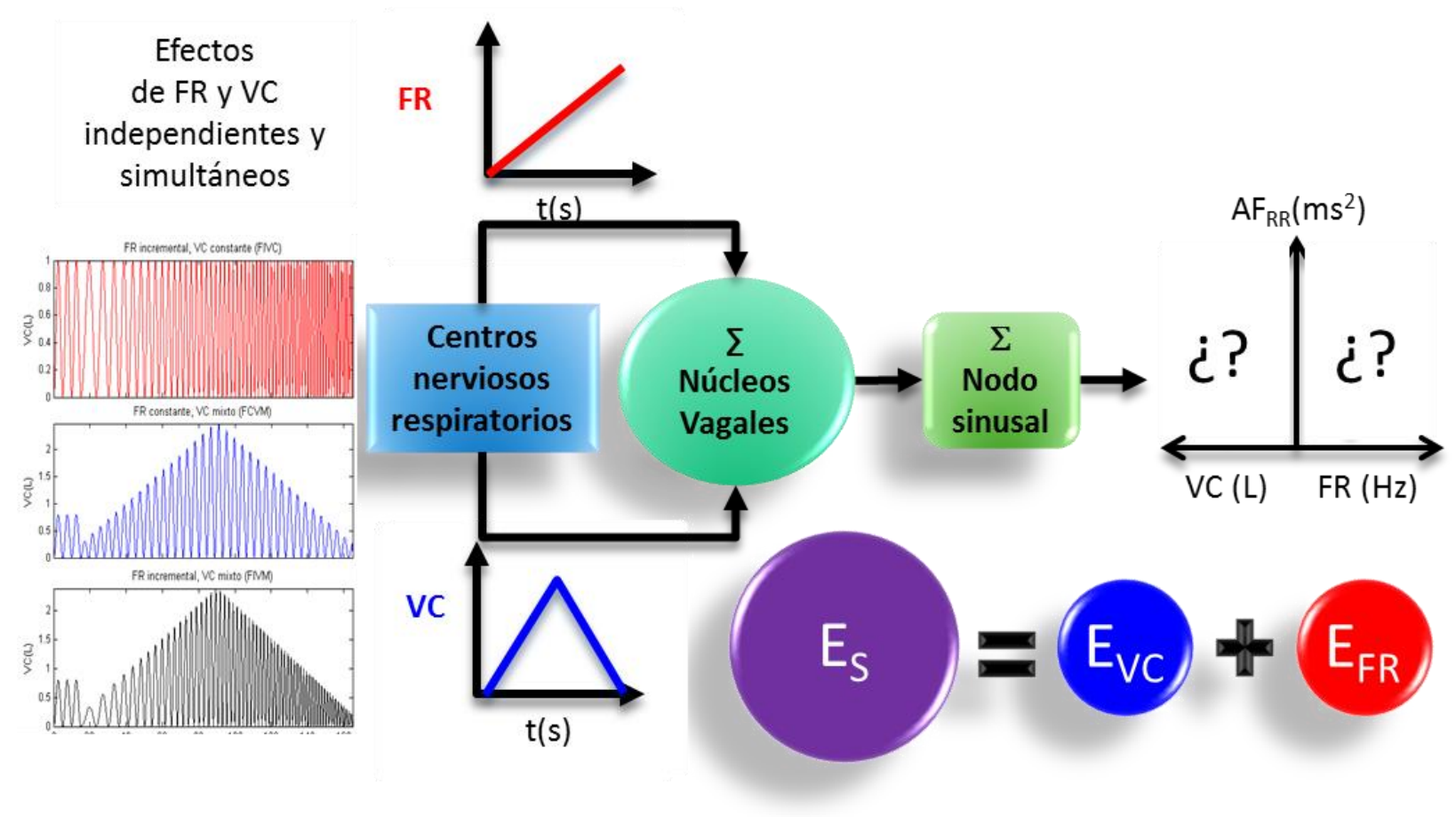

Fig. 15. Al comparar las respuestas provocadas sobre la potencia de $\mathrm{AF}_{\mathrm{RR}}$ por la variación continua y lineal de cada variable respiratoria por separado con los cambios durante su variación combinada exploraremos la manera en que el sistema núcleos vagales-nodo sinusal que genera la ASR integra dos aferencias que varían simultáneamente. 
2. Caracterizar el efecto de un protocolo de variación lineal de VC sobre la potencia del componente $\mathrm{AF}_{\mathrm{RR}}$, evaluando el tipo de relación que existe entre estas variables y si hay un límite en volumen de la ASR (Fig. 16A).

3. Examinar la relación $\mathrm{FIAF}_{\mathrm{RR}}-\mathrm{FR}$ para establecer el umbral de ruptura de la asociación entre las dos variables o límite en frecuencia de la ASR (Fig. 16B).

A

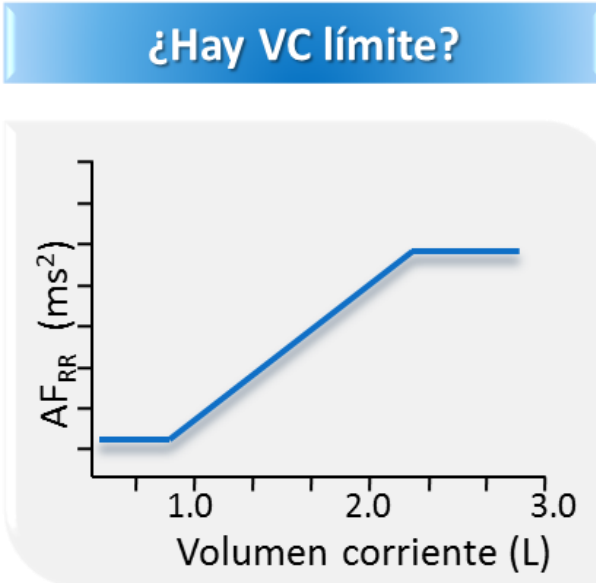

B
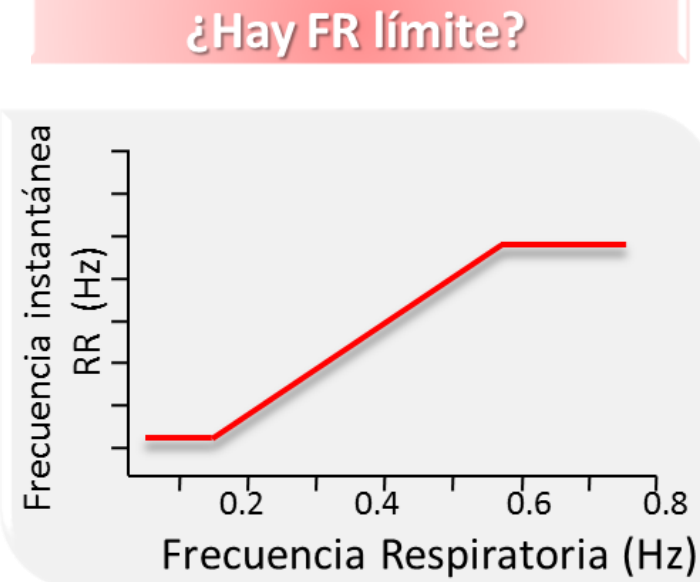

Fig. 16. La variación continua y lineal de las variables respiratorias $V C(A)$ y $F R(B)$ en un amplio rango de valores permitirá la evaluación del efecto que provoca sobre la amplitud de la ASR, así como la existencia de valores umbrales mínimos o máximos más allá de los cuales la ASR no responde.

4. Desarrollar un indicador de actividad vagal que (Fig. 17):

a. Considere el efecto simultáneo de las dos variables ventilatorias.

b. Evalúe si los cambios en el control autonómico-cardiovascular se deben a cambios en la respiración o a otra aferencia.

c. Se pueda superponer a otras maniobras para evaluar sus efectos autonómicocardiovasculares. Esto implica que debe ser de corta duración.

d. Sea formalmente manejable, es decir, de preferencia lineal y de fácil comparación. 


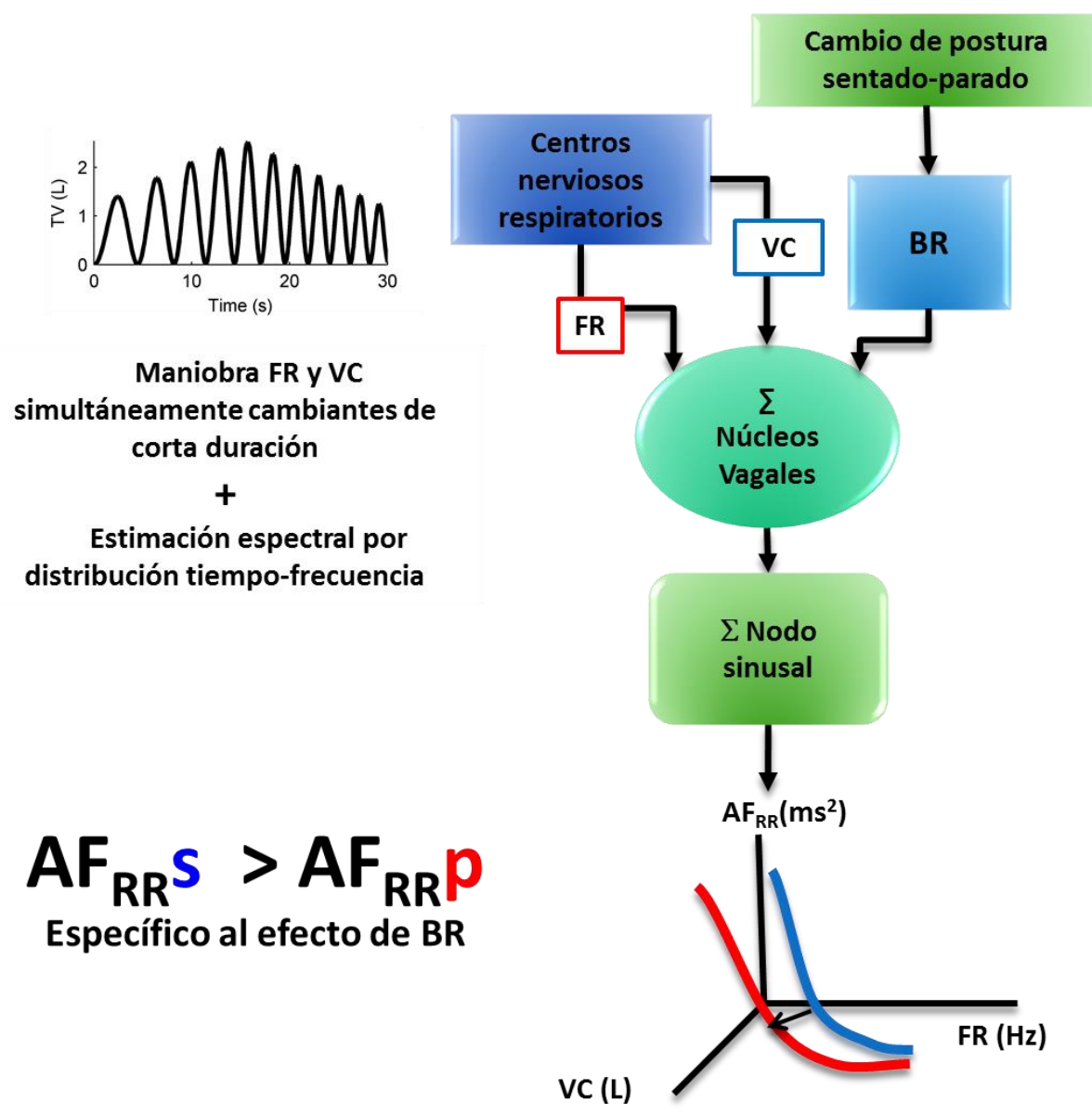

Fig. 17. Si se contara con una maniobra respiratoria que permitiera controlar simultáneamente los cambios de FR y VC en un amplio rango de valores durante un tiempo corto permitiría sobreponer este control respiratorio a la ejecución de otra maniobra fisiológica como el cambio de postura activo, reduciendo la ambigüedad en la interpretación de los indicadores espectrales de la VFC. Si se comparara la potencia de $A F_{R R}$ obtenida durante una maniobra de respiración controlada en posición sentada $\left(A F_{R R} S\right)$ con la obtenida mientras se repite la misma maniobra de respiración controlada pero ahora en posición de pie $\left(A F_{R R} P\right)$, se tendría la certeza de que las variaciones del indicador serían causadas específicamente por el efecto del barorreflejo (BR).

5. Examinar el efecto de las maniobras sobre la fracción espirada de $\mathrm{CO}_{2}\left(\% \mathrm{CO}_{2}\right)$.

6. Examinar si existen diferencias entre las respuestas de hombres y mujeres. 


\section{Etapa I: Pruebas piloto para el desarrollo de la metodología}

Para la consecución satisfactoria de los objetivos propuestos se plantearon dos estrategias metodológicas generales, consistentes:

1. Analizar las señales fisiológicas de interés con un comportamiento no estacionario mediante una técnica de análisis espectral capaz de seguir los cambios de los componentes espectrales de la VFC en condiciones no estacionarias.

2. Desarrollar maniobras de respiración controlada doblemente variantes (MRC2), en las cuales tanto la FR como el VC cambien de manera continua y lineal.

Cada una de estas estrategias involucró la ejecución de tareas metodológicas específicas.

\section{Verificación del desempeño de la DPWVS.}

Esta tarea se resolvió mediante la comparación de la frecuencia instantánea de una señal tipo chirp estimada por la DPWVS y por otro método apropiado para el análisis de señales no estacionarias, la DC (Kim y Euler 1997). Las frecuencias instantáneas estimadas por ambos métodos resultaron ser similares entre ellas y a la frecuencia esperada, aunque el amplio ancho de banda de la señal ocasionó que el resultado de la DC presentara varios artefactos indeseables (Fig. 18).

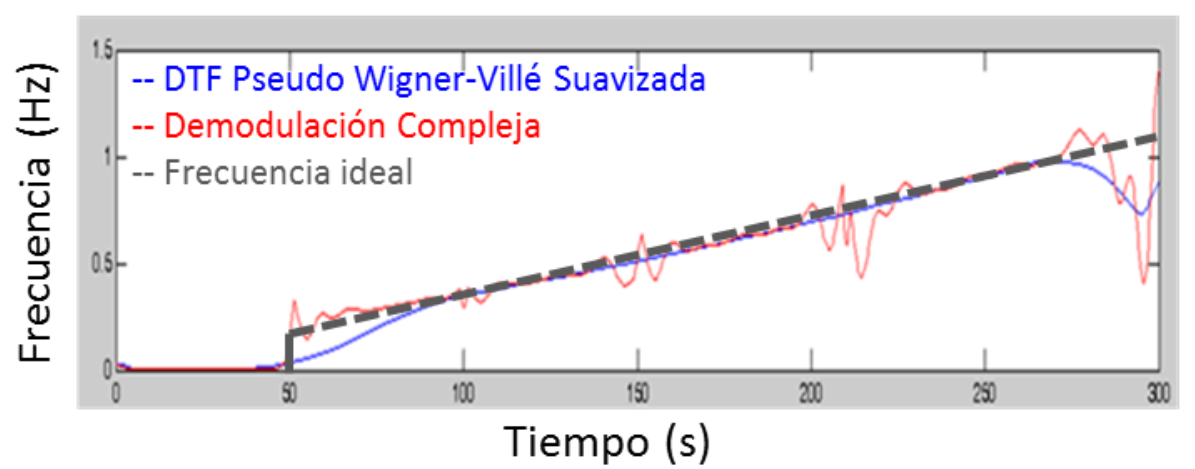

Fig. 18. Estimación, por dos métodos diferentes, de la frecuencia instantánea de una señal chirp. Se observa que ambos métodos (demodulación compleja y distribución Pseudo WignerVillé Suavizada) arrojan resultados muy similares, pero con algunos artefactos. 


\section{Selección y evaluación de MRC2}

\section{Tarea 1. Generar los patrones respiratorios visuales.}

Esta tarea fue resuelta utilizando dos computadoras equipadas con sendos sistemas MP150 (Biopac). En una de ellas se generaron los patrones respiratorios deseados empleando MatLab, los cuales fueron enviados a la otra computadora mediante una salida analógica (Fig. 19). La otra computadora recibió y desplegó simultáneamente la señal del estímulo respiratorio y la señal del neumotacómetro controlada por el sujeto, con la cual debía intentar sobrescribir el patrón desplegado, mientras adquiría y registraba las señales de ECG y $\% \mathrm{CO}_{2}$.

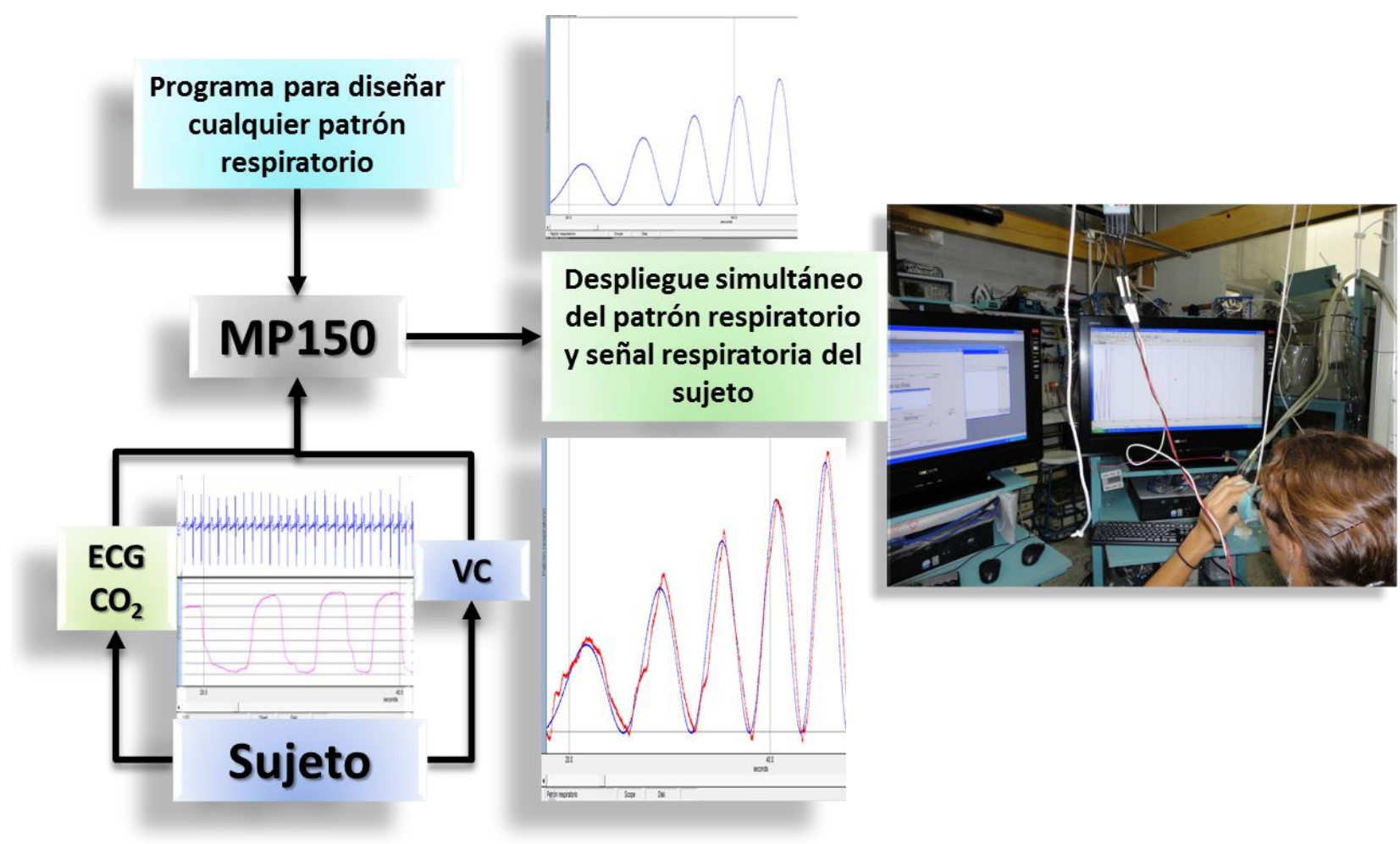

Fig. 19. Solución encontrada a la tarea de generar un patrón visual para el control respiratorio: el MP150 de Biopac ocupa un lugar central al desplegar de manera simultánea el patrón respiratorio generado de antemano, y registrar las señales respiratorias y cardiovasculares del sujeto.

\section{Tarea 2. Estudios piloto para definir las características relevantes de los protocolos respiratorios definitivos}

A fin de construir protocolos de control respiratorio que pudieran ser ejecutados por cualquier individuo sano, se pusieron a prueba ocho protocolos piloto generados empleando características 
consideradas de interés: duración de la maniobra, rangos de FR y VC, y dirección de cambio de las dos variables (incremento/decremento).

\section{Metodología}

\section{Sujetos}

Todos los protocolos fueron probados en una muestra de sujetos que cumplieran con los criterios de inclusión deseados para la muestra definitiva: jóvenes (de entre 18 y 25 años de edad), no fumadores, normotensos, con ECG y espirometría normales y sedentarios. En total se estudiaron 11 voluntarios, para analizar los efectos fisiológicos de las posibles combinaciones de variación individual o simultánea de FR y VC.

\section{Conjunto piloto de maniobras de control respiratorio}

Los primeros protocolos piloto generados tuvieron las siguientes características: $60 \mathrm{~s}$ de duración, incrementos o decrementos lineales de la FR en el rango de 0.1 y $0.6 \mathrm{~Hz}$, a razón de $0.0083 \mathrm{~Hz} / \mathrm{s}$, incrementos o decrementos lineales del VC entre 0.25 y 3 litros, a razón de $0.0458 \mathrm{~L} / \mathrm{s}$. Para advertir al sujeto del inicio de la maniobra, los patrones comenzaban con tres ondas de $\mathrm{FR}=0.2$ $\mathrm{Hz}$ y $\mathrm{VC}=0.8 \mathrm{~L}$.

Maniobras univariantes (Fig. 20):

FIVC: Frecuencia Incremental $(0.1$ a $0.6 \mathrm{~Hz})$, Volumen Constante (1 L hombres, $0.8 \mathrm{~L}$ mujeres).

FDVC: Frecuencia Decreciente $(0.6$ a $0.1 \mathrm{~Hz})$, Volumen Constante (1 L hombres, $0.8 \mathrm{~L}$ mujeres).

FCVI: Frecuencia Constante $(0.2 \mathrm{~Hz})$, Volumen Incremental $(0.25$ a $3 \mathrm{~L})$.

FCVD: Frecuencia Constante (0.2 Hz), Volumen Decreciente (3 a 0.25 L).
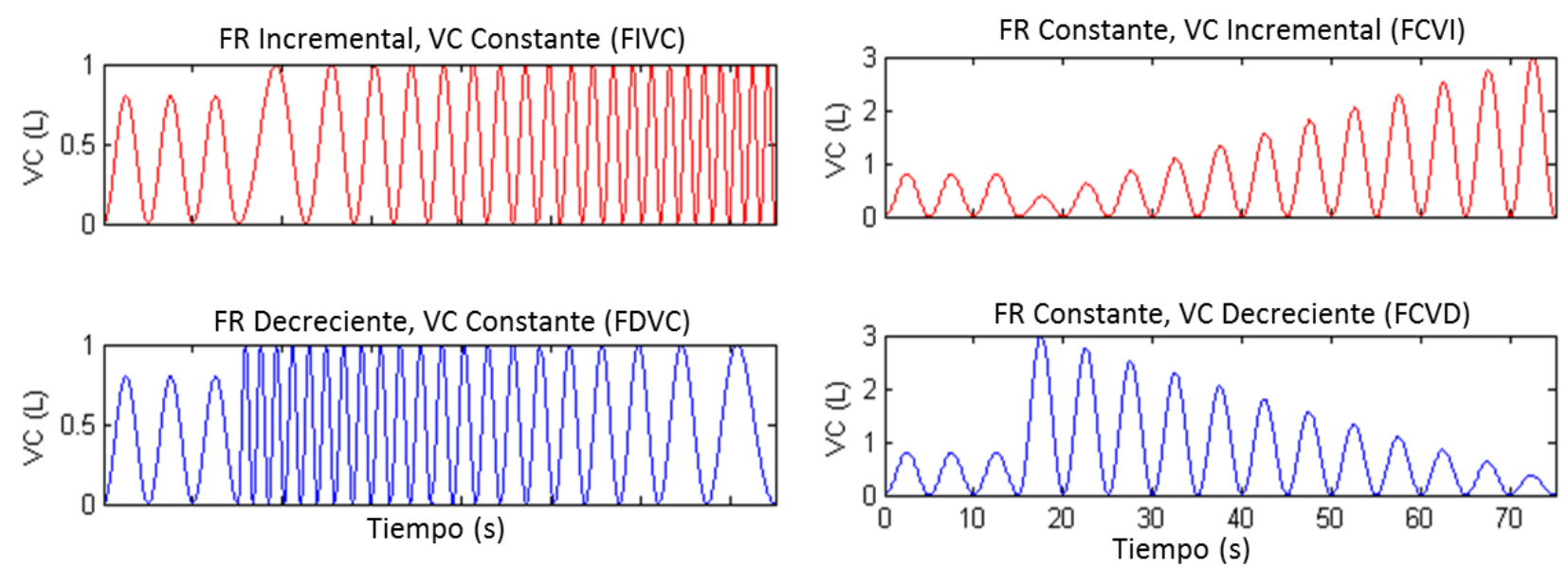

Fig. 20. Maniobras de control respiratorio donde una de las variables permanece constante mientras la otra cambia de manera lineal, creciente o decreciente. Las tres ondas del inicio de cada maniobra alertan al sujeto del inicio del patrón. 
Maniobras doblemente variantes (Fig. 21)

1. FIVD: Frecuencia Incremental, Volumen Decreciente.

2. FDVI: Frecuencia Decreciente, Volumen Incremental.

3. FIVI: Frecuencia Incremental, Volumen Incremental.

4. FDVD: Frecuencia Decreciente, Volumen Decreciente.
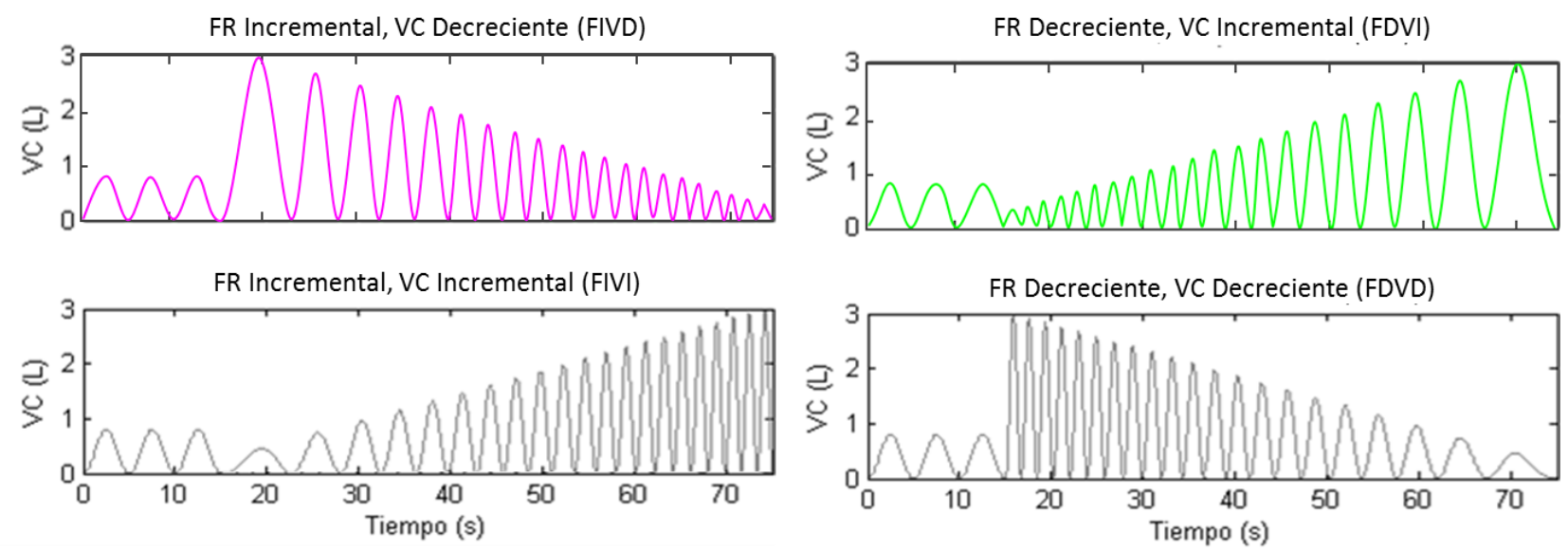

Fig. 21. Maniobras de control respiratorio doblemente variantes. Las tres ondas del inicio de cada maniobra alertan al sujeto del inicio del patrón. Las maniobras donde las dos variables respiratorias cambiaban en dirección paralela no pudieron ser realizadas por ningún sujeto, así que fueron descartadas.

\section{Registro y procesamiento de señales}

Durante la ejecución de los protocolos de respiración controlada registramos el ECG y el respirograma de cada sujeto, a partir de los cuales se detectaron las ondas $\mathrm{R}$ y los máximos de cada ciclo respiratorio para formar el tacograma y la serie de amplitud de VC. Las series de intervalos RR y VC fueron procesadas mediante la DPWVS para calcular la potencia y frecuencia instantánea de sus componentes espectrales en la banda de la FR. Se aplicó una transformación logarítmica sobre el componente de $\mathrm{AF}_{\mathrm{RR}}\left(\ln \mathrm{AF}_{\mathrm{RR}}\right)$ para normalizar su distribución.

\section{Resultados de los estudios piloto}

Se evaluaron la dificultad de ejecución de los diferentes protocolos propuestos y el grado de asociación lineal existente entre el $\ln \mathrm{AF}_{\mathrm{RR}}$, la $\mathrm{FR}$ y el VC. Las gráficas de las relaciones $\ln \mathrm{AF}_{\mathrm{RR}^{-}}$ FR y $\ln \mathrm{AF}_{\mathrm{RR}}-\mathrm{VC}$ presentadas en las Figs. 22 y 23 se formaron con los promedios ensamblados de las relaciones individuales. Las gráficas de barras muestran la comparación de las pendientes y los coeficientes de correlación promedio de las regresiones individuales. En la Fig. 22 se encuentran los resultados obtenidos por la realización de las maniobras en las que sólo una de las variables 
ventilatorias cambia mientras la otra permanece constante. En general, los sujetos no reportaron mayor dificultad en la ejecución de estas maniobras respiratorias.
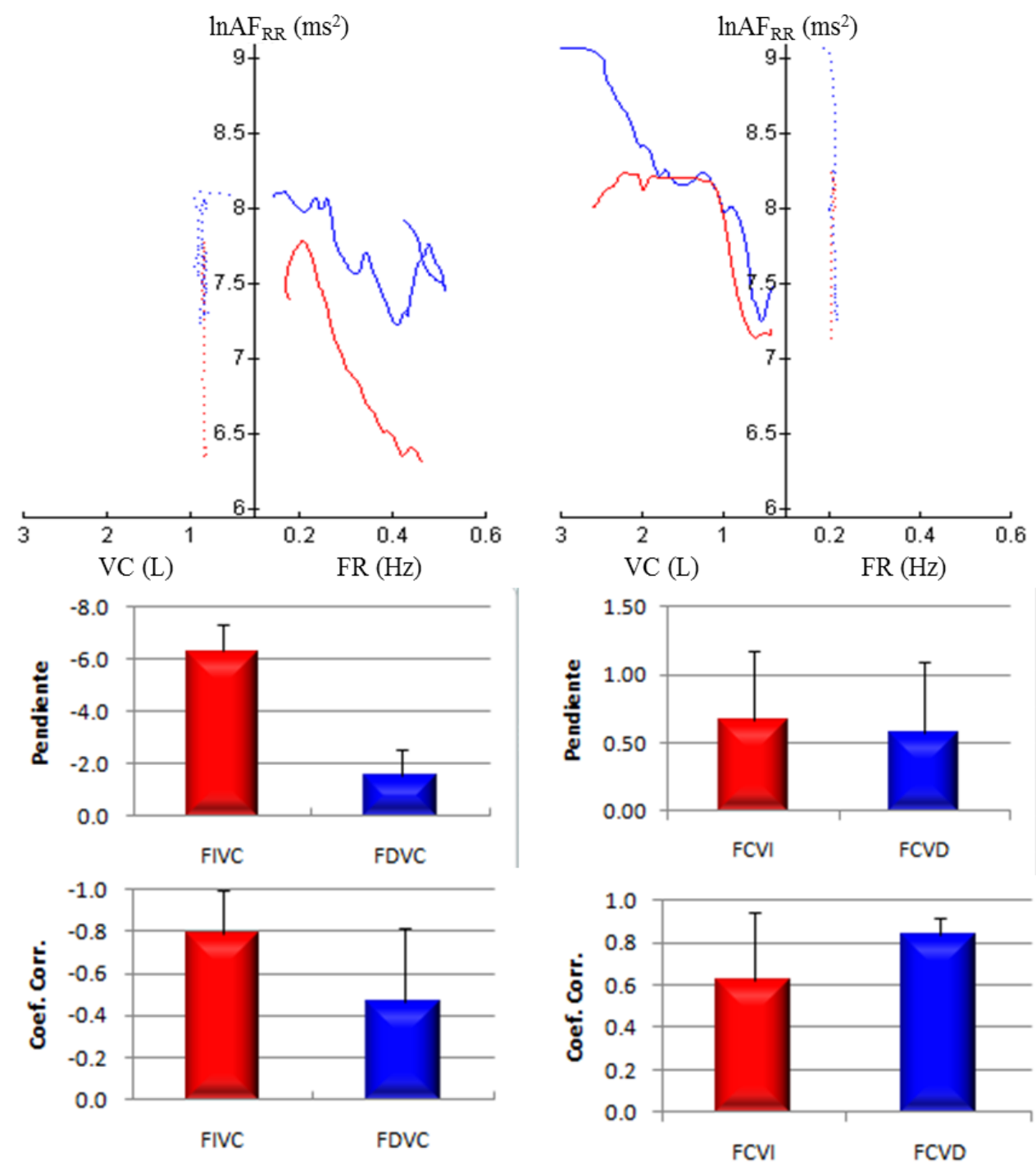

Fig. 22. Resultados de los protocolos respiratorios univariantes. En la fila superior se observan las relaciones

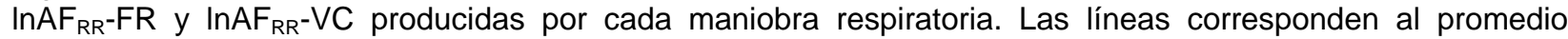
ensamblado de las respuestas individuales. Las gráficas de barras muestran la comparación de las pendientes y los coeficientes de correlación de cada relación. Las relaciones $\ln A F_{R R}-F R$ y $\ln A F_{R R}-V C$ obtenidas por los protocolos FIVC y FCVD presentaron mayores coeficientes de correlación aquéllas de sus contrapartes FDVC y FCVI. Estas diferencias indican que el sistema estudiado presenta histéresis.

En respuesta a las maniobras FIVC y FCVD, las relaciones $\ln \mathrm{AF}_{\mathrm{RR}}-\mathrm{FR}$ y $\ln \mathrm{AF}_{\mathrm{RR}}-\mathrm{VC}$ presentaron elevados coeficientes de correlación lineal $(r>0.8, \mathrm{p}<0.001)$. En las dinámicas de los promedios ensamblados se aprecia que la relación $\ln \mathrm{AF}_{\mathrm{RR}}-\mathrm{FR}$ presenta un segmento muy lineal entre $0.15 \mathrm{y}$ $0.55 \mathrm{~Hz}$. En contraste, las relaciones $\ln \mathrm{AF}_{\mathrm{RR}}-\mathrm{FR}$ y $\ln \mathrm{AF}_{\mathrm{RR}}-\mathrm{VC}$ mostraron menores coeficientes de correlación en respuesta a los estímulos FDVC y FCVI $(\mathrm{r} \leq 0.6, \mathrm{p}<0.05)$. La relación $\ln \mathrm{AF}_{\mathrm{RR}}-\mathrm{VC}$ durante la maniobra FCVI fue linealmente creciente como se esperaba entre 0.25 y 1 L, pero 
permaneció constante después de este último VC. El hecho de que las respuestas de los protocolos crecientes fueran diferentes a los decrecientes indica la existencia de histéresis asociada a la dirección del cambio de las variables respiratorias.

Los resultados obtenidos de la realización de las maniobras donde las dos variables ventilatorias cambian simultáneamente en direcciones opuestas o paralelas se muestran en la Fig. 23.
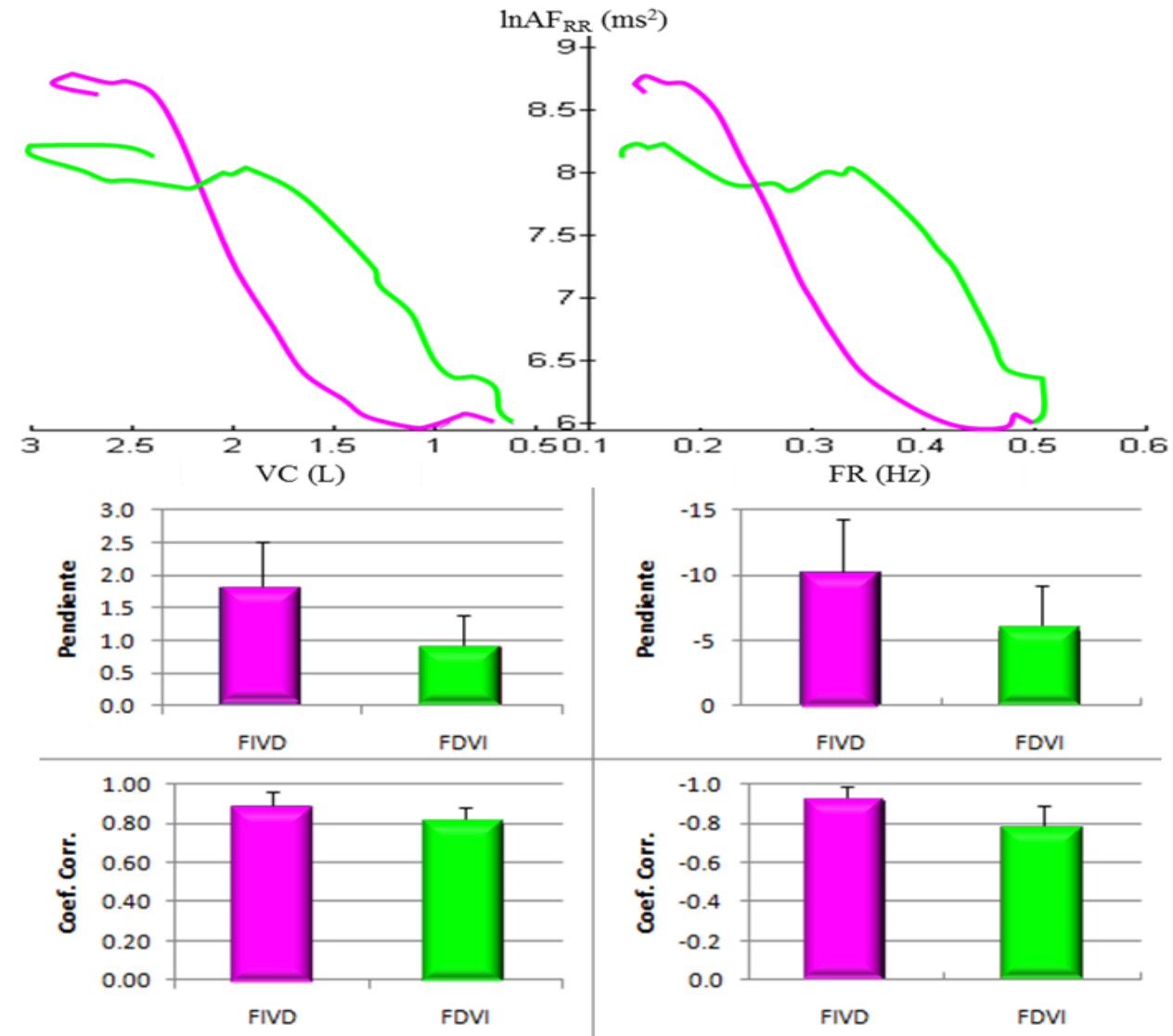

Fig. 23. Resultados de los protocolos respiratorios doblemente variantes. En la parte superior se observan los promedios ensamblados de las relaciones $\ln A F_{R R}-F R$ y $\ln A F_{R R}-V C$ producidas por cada maniobra respiratoria. Las gráficas de barras de la parte inferior muestran la comparación de las pendientes y los coeficientes de correlación de cada relación. Como los sujetos fueron incapaces de concluir los protocolos donde ambas variables cambiaban en dirección paralela (FIVI y FDVD) sólo se reportan los resultados de los protocolos FIVD y FDVI. Ambos produjeron respuestas lineales en el $\operatorname{InAF}_{R R},(r>0.8, p<0.01)$, aunque con diferentes pendientes $(p>0.05)$. La relación InAF $F_{R R}-V C$ volvió a presentar un umbral ante la variación creciente del VC.

Como los sujetos fueron incapaces de ejecutar exitosamente los protocolos donde la FR y el VC variaban paralelamente, sólo se presentan los resultados correspondientes a los protocolos FIVD y FDVI. También hubo diferencias en las respuestas a estos protocolos, evidenciando la existencia de histéresis aun cuando ambas variables ventilatorias cambian simultáneamente. Todas las relaciones presentaron coeficientes de correlación cercanos a 0.8. El efecto del VC incremental del 
protocolo FDVI ocasionó que las relaciones $\ln \mathrm{AF}_{\mathrm{RR}}-\mathrm{FR}$ y $\ln \mathrm{AF}_{\mathrm{RR}}-\mathrm{VC}$ presentara un cambio de pendiente alrededor de $1.8 \mathrm{~L}$.

Estos resultados sirvieron como base para la construcción de nuevas maniobras de control respiratorio que condujeran a la consecución de los objetivos específicos planteados. Dado que resulta mucho más sencillo manejar y comparar relaciones lineales que cualquier otro tipo de relación, al poder ser representadas mediante dos únicos parámetros (pendiente e intercepto), el criterio de selección de las características de los protocolos fue encontrar las combinaciones que produjeran las relaciones $\ln \mathrm{AF}_{\mathrm{RR}}-\mathrm{FR}$ y $\ln \mathrm{AF}_{\mathrm{RR}}-\mathrm{VC}$ con los mayores coeficientes de correlación. Por esta razón, se seleccionaron la FR incremental y el VC decreciente tanto para los protocolos univariantes como los doblemente variantes.

Para evaluar los efectos fisiológicos de la variación simultánea de las variables ventilatorias se generaron tres maniobras de mayor duración (150 s), dos univariantes y la tercera doblemente variante. El primer protocolo largo, abreviado FIVCL, consiste en el incremento lineal de la FR en el rango de 0.1 a $0.7 \mathrm{~Hz}$ a $\mathrm{VC}$ constante, $1 \mathrm{~L}$ para hombres y 0.8 para mujeres. El segundo protocolo, FCVDL consiste en el decremento lineal del VC en el rango de 3 a $0.25 \mathrm{~L}$ para hombres y de 2.5 a 0.25 L para mujeres, a una FR constante de $0.2 \mathrm{~Hz}$. El tercer protocolo, FIVDL consiste en la variación simultánea de ambas variables, FR incremental y VC decreciente. Estos tres protocolos fueron realizados en posición sentada y en orden aleatorio (Fig. 24).
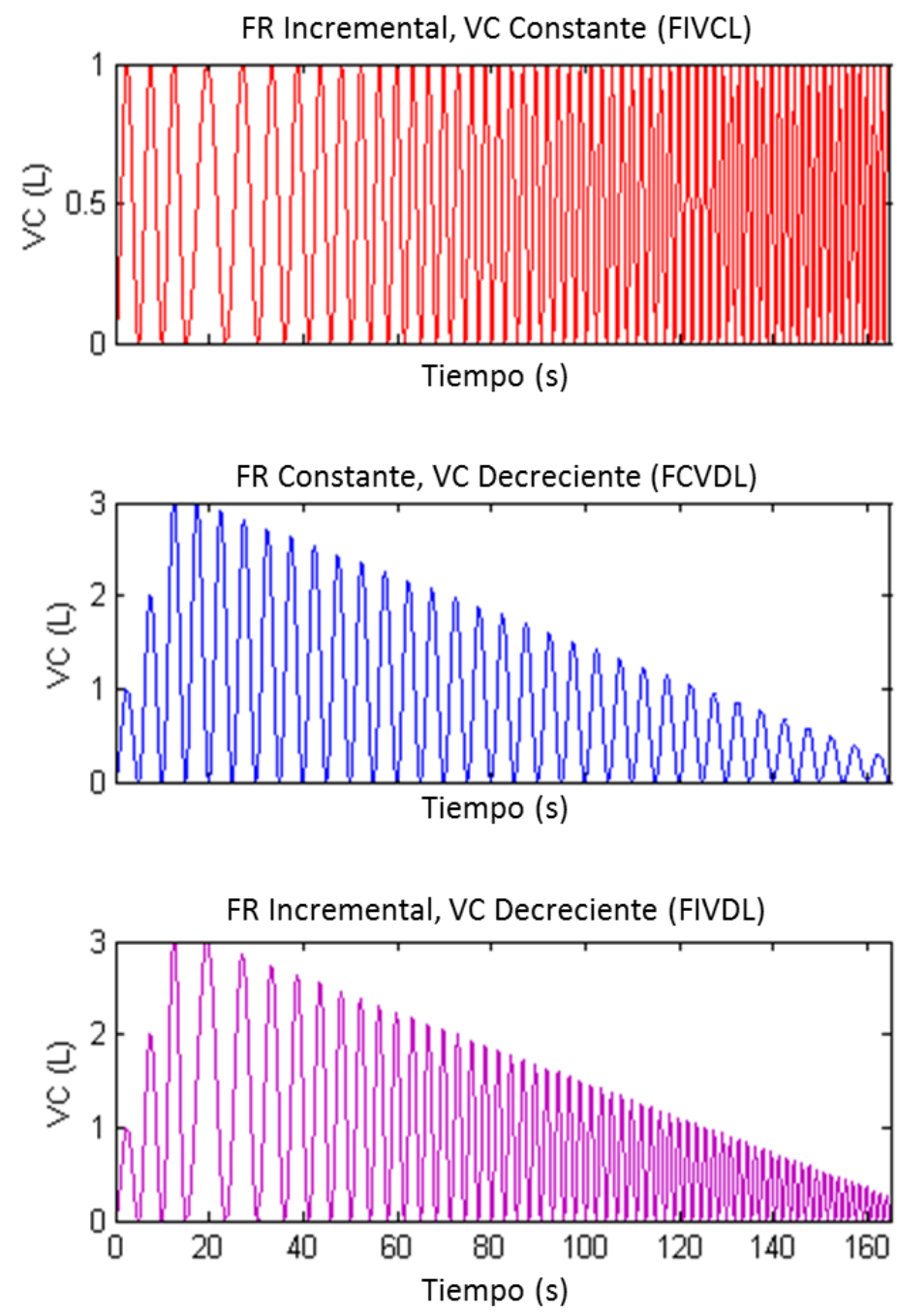

Fig. 24. Maniobras de control respiratorio para la evaluación fisiológica. 

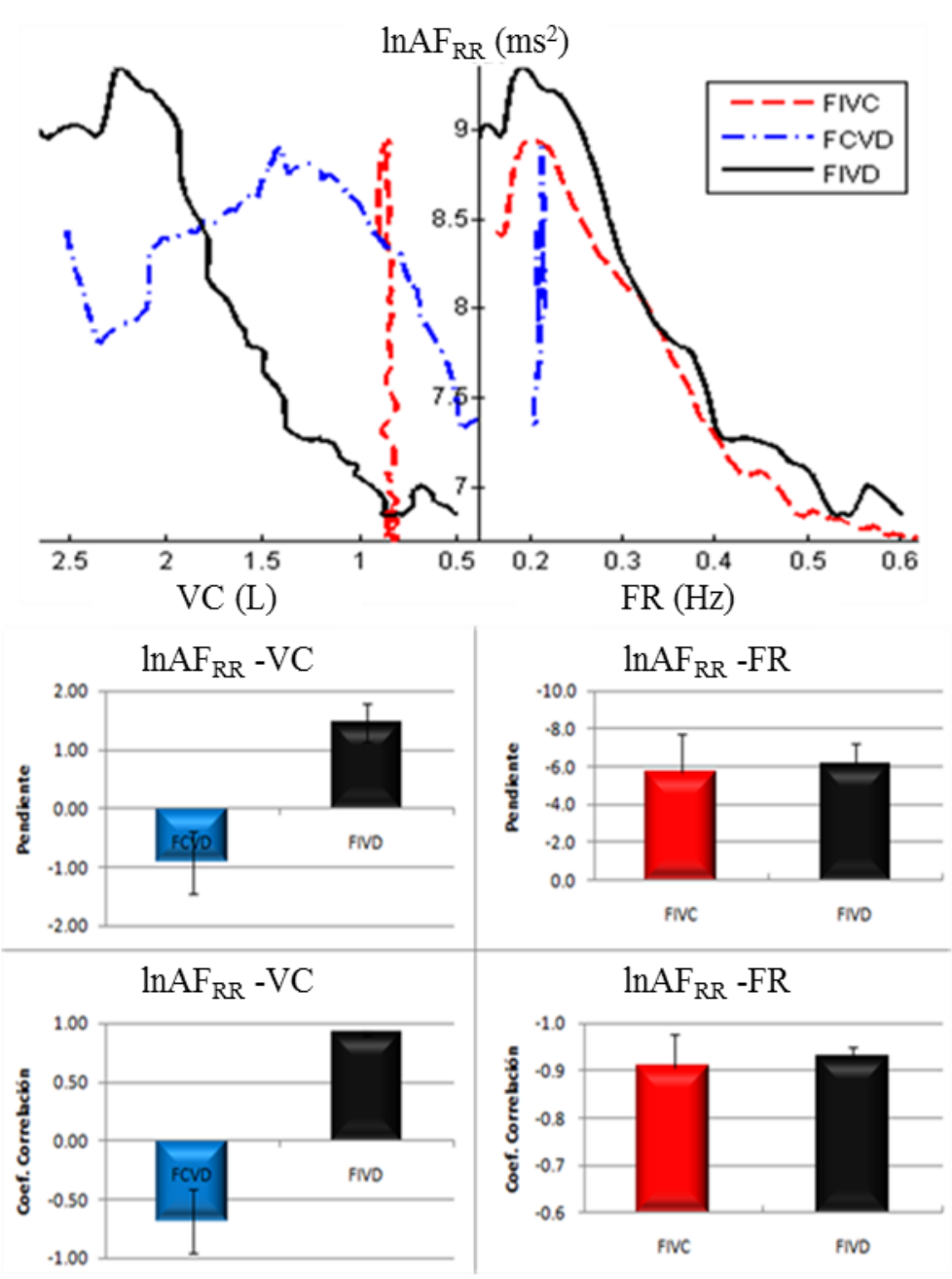

Fig. 25. Protocolos respiratorios ampliados para evaluación fisiológica. En la parte superior se observa la comparación entre $\ln A F_{R^{2}}-F R$ y $\ln A F_{R R}-V C$ producidas por cada maniobra respiratoria. Las líneas corresponden al promedio ensamblado de las respuestas individuales. Las gráficas de barras de la parte inferior muestran la comparación de las pendientes y los coeficientes de correlación de cada relación. La relación $I n A F_{R R}-F R$ no presentó diferencias $(p>0.05)$ entre el protocolo univariante para la FR (FIVC) y el protocolo doblemente variante (FIVD). En respuesta al protocolo FCVD, la relación InAF $F_{R R}-V C$ presentó un fenómeno de umbral en $1.5 \mathrm{~L}$, pero al variar simultáneamente las dos variables ventilatorias (FIVD) se produjo una respuesta en la que dominó la influencia de la FR.
Como puede apreciarse en la Fig. 25, la relación $\ln \mathrm{AF}_{\mathrm{RR}}-\mathrm{FR}$ fue lineal en los dos protocolos $(r>0.9, \mathrm{p}<0.001)$. Las pendientes, los interceptos y los coeficientes de correlación del protocolo univariante para la FR (FIVCL) y del protocolo doblemente variante (FIVDL) no fueron estadísticamente diferentes ( $\mathrm{p}>0.05)$. La relación $\ln \mathrm{AF}_{\mathrm{RR}}-\mathrm{VC}$ mostró un comportamiento bifásico en respuesta al protocolo FCVD: la amplitud del $\ln \mathrm{AF}_{\mathrm{RR}}$ aumentó conforme la amplitud del VC decrecía hasta alcanzar un punto máximo alrededor de $1.5 \mathrm{~L}$, posterior al cual disminuyó proporcionalmente al VC. Este fenómeno de umbral indica un valor óptimo en el cual la influencia del VC sobre la ASR es máxima, hallazgo que no ha sido reportado previamente. Más aún, se contrapone a la concepción generalizada de que la amplitud de la ASR es directamente proporcional a la amplitud del VC. El comportamiento del $\ln \mathrm{AF}_{\mathrm{RR}}$ en respuesta a la maniobra doblemente cambiante (FIVD) fue lineal en la mayor parte del rango abarcado, aunque volvió a presentar el fenómeno de umbral, pero en esta ocasión el máximo se alcanzó entre los 2.5 y 2 L. El efecto de la variación simultánea de la FR y VC sobre la relación $\ln \mathrm{AF}_{\mathrm{RR}}-\mathrm{VC}$ es complejo, ya que, a pesar de incluir valores similares de VC y FR, los valores de $\ln \mathrm{AF}_{\mathrm{RR}}$ son diferentes. 
Para generar el indicador vagal que permita mantener constante la influencia de las dos variables ventilatorias sobre la salida vagal mientras se realiza otra maniobra, fue necesario reducir la duración a 30 s y ajustar los rangos de variación de FR y VC a los valores donde el control fuera más sencillo de realizar y la respuesta vagal fuera más lineal: de 0.2 a $0.5 \mathrm{~Hz}$ y de 2.0 a $0.5 \mathrm{~L}$ respectivamente. De esta manera se generaron dos protocolos univariantes (FIVC y FCVD) y uno doblemente variante (FIVD), cada uno de los cuales fue ejecutado en dos posturas, sentado y parado (Fig. 26).
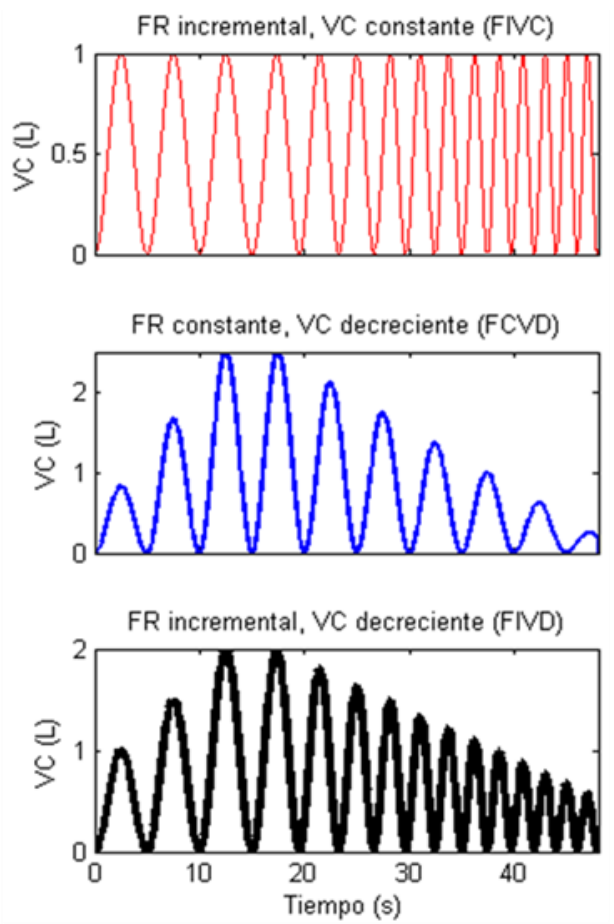

Fig.26. Protocolos para Indicador vagal. Las maniobras respiratorias de corta duración desarrolladas fueron aplicadas en dos posturas, sentado (s) y parado $(p)$.
Las relaciones $\ln \mathrm{AF}_{\mathrm{RR}}-\mathrm{FR}$ de la maniobra doblemente variante (FIVD) resultaron lineales y no fueron diferentes a las correspondientes a la maniobra individual (FIVC). Las relaciones $\ln \mathrm{AF}_{\mathrm{RR}}-\mathrm{VC}$ fueron no lineales en respuesta a la maniobra FCVD pero se linealizaron durante la maniobra doblemente variante (FIVD). En todos los casos, las pendientes y los coeficientes de correlación de las dos relaciones fueron menores en la postura parada que en sentada (Fig. 27). Además, después de una caída inicial en $\ln \mathrm{AF}_{\mathrm{RR}}$ debido al cambio de postura activo, ocurrió una recuperación seguida de otra disminución. Puesto que la respiración se mantuvo controlada, estos efectos son atribuibles íntegramente a la compleja dinámica vagal producida por el cambio de postura, la cual consiste en un retiro vagal inicial para compensar la caída inicial de la presión arterial, seguido de un aumento en la salida vagal para frenar la elevación de la presión. 
Las regresiones múltiples computadas para las maniobras combinadas no fueron confiables dado que, al hacer que tanto la FR como el VC variaran linealmente, ocasionamos que nuestras variables predictivas fueran multicolineales, es decir, que existiera entre ellas un alto grado de correlación. Para garantizar la no colinealidad de las variables predictivas, diseñamos un nuevo conjunto de maniobras respiratorias, en las cuales la FR aumenta linealmente mientras el VC cambia de manera mixta, incrementándose linealmente durante la primera mitad de la maniobra y reduciéndose durante la segunda mitad. De esta manera, la relación VC-FR no presenta correlación significativa y es posible calcular un plano de regresión múltiple confiable que estime los valores del $\ln \mathrm{AF}_{\mathrm{RR}}$ en términos de VC y FR simultáneamente. Se generaron entonces dos juegos de nuevos

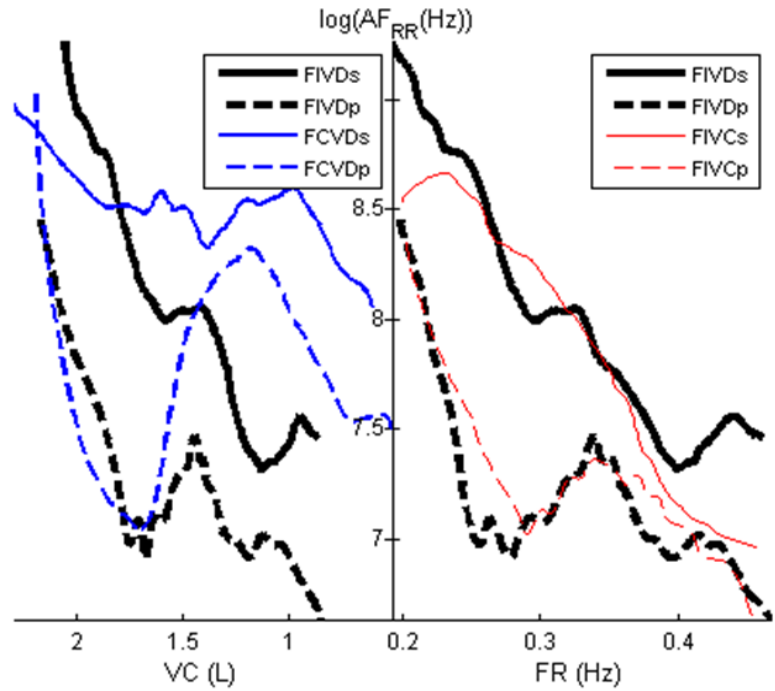

In(AFRR) vs. VC
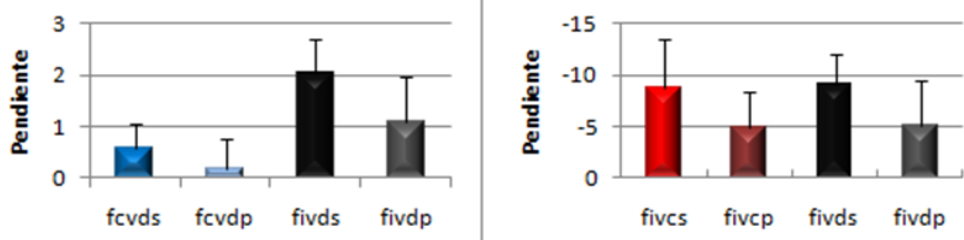

In(AFRR) vs. VC

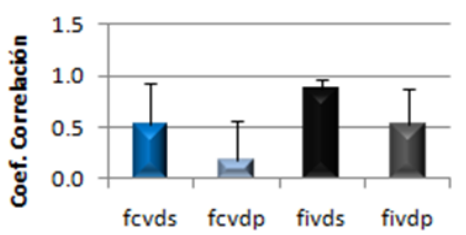

In(AFRR) vs. FR

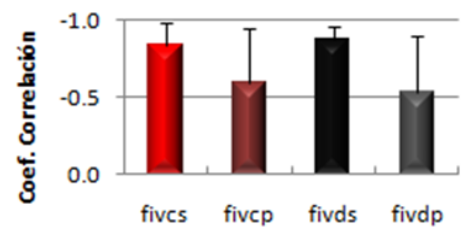

Fig.27. En la parte superior se muestran los patrones ensamblados de las relaciones $\ln _{n} F_{R R}-F R$ y $\operatorname{InAF}_{R R}-V C$ resultantes de aplicar las tres maniobras en las dos posturas, sentado (s) y parado ( $p$ ). Las gráficas de barras ubicadas en la parte inferior muestran la comparación de las pendientes y los coeficientes de correlación de las tres maniobras en las dos posturas. Las relaciones $\ln A F_{R R}-F R$ de la maniobra doblemente variante (FIVD) no fueron diferentes a las correspondientes a la maniobra individual (FIVC). Las relaciones $\ln A F_{R R}-V C$ fueron no lineales en respuesta a la maniobra FCVD pero se linealizaron durante la maniobra doblemente variante (FIVD). En todos los casos las pendientes y los coeficientes de correlación de las dos relaciones fueron menores en parado que en sentado, efectos atribuibles íntegramente a la compleja dinámica vagal producida por el cambio de postura.

patrones respiratorios, unos de larga duración (150 s) y rangos amplios (FR, de 0.1 a 0.7 Hz, VC, de 3 a 0.25 L), empleados para analizar los efectos fisiológicos de la variación simultánea de la FR y el VC; y otros de corta duración (30s) y rangos restringidos (FR de 0.2 a $0.5 \mathrm{~Hz}, \mathrm{VC}$ de 1 a 2.5 L) a los que se les sobrepondrá el cambio de postura activo. Cada conjunto de protocolos incluye 
una maniobra en la cual la FR se incrementa continuamente mientras el VC permanece constante $\left(\mathrm{FR}_{\mathrm{LI}}\right.$ ), otra donde la FR permanece constante a $0.2 \mathrm{~Hz}$ mientras el VC varía de manera mixta, primero incrementándose y luego decreciendo linealmente $\left(\mathrm{VC}_{\mathrm{LI}}-\mathrm{VC}_{\mathrm{LD}}\right)$ y una maniobra doblemente variante, donde la FR se incrementa lineal y continuamente mientras el VC cambia de manera mixta $\left(\mathrm{FR}_{\mathrm{L}} / \mathrm{VC}_{\mathrm{LI}^{-}} \mathrm{VC}_{\mathrm{LD}}\right)$.

La realización de los estudios piloto permitió:

1. Poner a punto el arreglo de la instrumentación para el registro y adquisición de las señales fisiológicas ECG, volumen respiratorio y presión arterial no invasiva durante la realización de los diferentes protocolos de control respiratorio propuestos.

2. Elaborar y perfeccionar las herramientas necesarias para el análisis de las señales fisiológicas, consistentes en:

a. detección de puntos fiduciales (ondas R, presiones sistólica y diastólica, máximos de volumen respiratorio) y formación de series de tiempo

b. distribución de tiempo-frecuencia y cálculo de indicadores espectrales

c. formación de relaciones entre indicadores espectrales, generación de gráficas tipo Margaria (1976) y ajuste de curvas.

3. Identificar los efectos fisiológicos de las posibles combinaciones de estímulos y seleccionar las características que permitan la ejecución eficiente de los protocolos de control respiratorio doblemente variante para lograr los objetivos planteados. 


\section{Etapa II: Aplicación experimental de la metodología desarrollada}

\section{Sujetos}

Treinta y ocho residentes del DF (altitud de $2240 \mathrm{~m}$ ) participaron voluntariamente en esta investigación. Los criterios de inclusión fueron: sanos (estado de salud establecido por examinación clínica SCS, ECG de reposo y espirometría), jóvenes, normotensos, sin sobrepeso (índice de masa corporal $<25 \mathrm{~kg} / \mathrm{m}^{2}$ ), no fumadores y sedentarios (Tabla 1). Se les pidió que, durante las 12 horas previas al estudio, se abstuvieran de ingerir grandes cantidades de comida, bebidas alcohólicas o estimulantes y de realizar actividad física intensa. Todos los voluntarios presentaron su consentimiento informado por escrito (Anexo 1).

Tabla 1. Medidas antropométricas de los voluntarios estudiados

\begin{tabular}{cccccc}
\hline & $\mathrm{N}$ & Edad (años) & Estatura $(\mathrm{cm})$ & Peso $(\mathrm{kg})$ & $\begin{array}{c}\text { Índice de masa } \\
\text { corporal }\left(\mathrm{kg} / \mathrm{m}^{2}\right)\end{array}$ \\
\hline Mujeres & 18 & $20.7 \pm 1.7$ & $158.4 \pm 5.8$ & $59.8 \pm 10.1$ & $23.8 \pm 3.4$ \\
Hombres & 20 & $21.3 \pm 1.8$ & $171.8 \pm 7.6$ & $71.3 \pm 15.4$ & $23.9 \pm 3.7$ \\
\hline
\end{tabular}

\section{Protocolo}

Cada sujeto realizó tres visitas al laboratorio. En la primera, se le informó exhaustivamente sobre el motivo, los objetivos, la metodología y los riesgos del protocolo experimental en que estaba invitado a participar. Si decidía participar, se le solicitaba su consentimiento informado por escrito, en concordancia con la Declaración de Helsinki que norma la experimentación en seres humanos. A continuación, se evaluaba el estado de salud del sujeto y, de cumplir todos los criterios de inclusión, se le entrenaba en la ejecución de las diferentes maniobras respiratorias para garantizar que las realizara correctamente.

En la segunda visita, el sujeto realizaba las maniobras respiratorias para la evaluación fisiológica en posición sentada, en orden aleatorio y con periodos de 5 minutos de reposo entre maniobras para permitir que su estado fisiológico regresara al nivel basal.

En la tercera visita el sujeto ejecutaba las maniobras respiratorias para el indicador vagal en orden aleatorio, primero en posición sentada y, después de un periodo de descanso de 5 minutos, la repetía en posición parada. La ejecución de la maniobras fue guiada visualmente mediante el 
sobrelapamiento de la señal respiratoria del sujeto y el patrón respiratorio en un monitor. Las señales de ECG, VC y perfil espiratorio de $\mathrm{CO}_{2}$ fueron registradas a lo largo de todo el protocolo. Los sujetos fueron estudiados entre las 10:00 y las 13:00 horas. La presión barométrica media en el laboratorio fue $591 \pm 2 \mathrm{mmHg}$.

\section{Maniobras de respiración controlada}

Cada maniobra para el control respiratorio constó de tres etapas (Fig. 28):

1. Control, consistente en tres respiraciones controladas a una FR de $0.2 \mathrm{~Hz}$ y a VC constante;

2. Maniobra, variación lineal y continua de una o ambas variables respiratoria, de 150 ó $30 \mathrm{~s}$ de duración; y

3. Recuperación, respiración libre de 10 s de duración.

Puesto que la capacidad vital y el VC de las mujeres son usualmente $20 \%$ menores que aquéllos de los hombres (Hankinson et al. 1999), se emplearon diferentes valores de VC para cada sexo, a fin de asegurar que el esfuerzo respiratorio fuera similar para todos los sujetos.

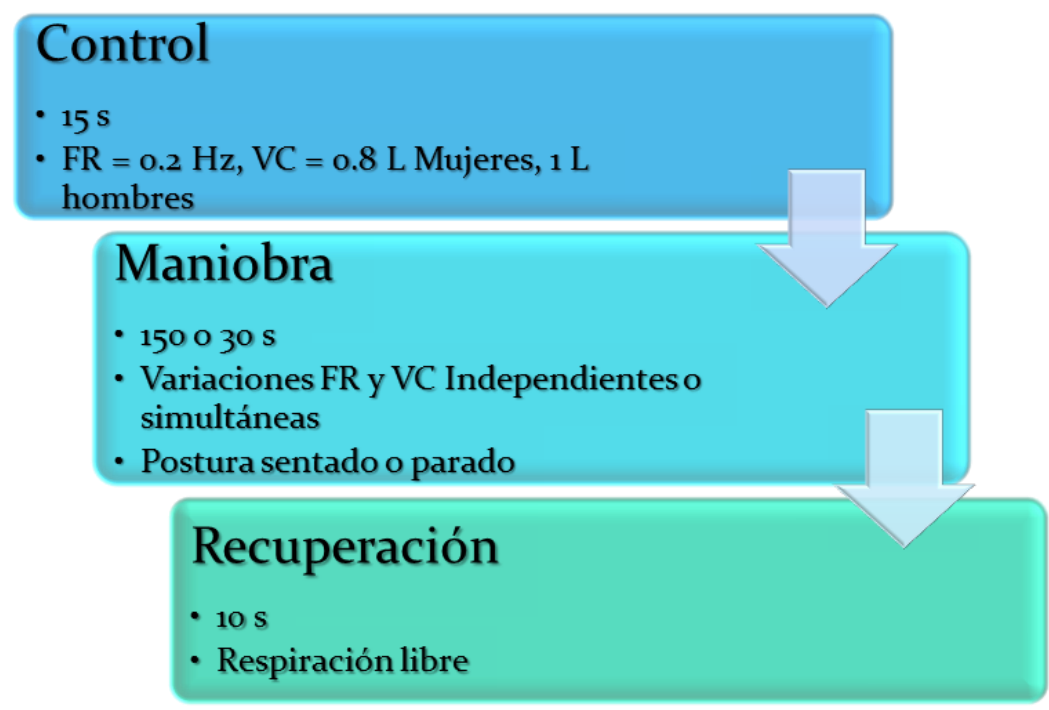

Fig.28. Estructura general de las maniobras de respiración controlada diseñadas. Todas las maniobras constaron de tres etapas: control, respiración controlada y recuperación, con características variantes de acuerdo al experimento realizado. Las características de las maniobras respiratorias fueron definidas con base en los resultados de los estudios piloto (págs. 26-37) y de la retroalimentación recibida en el examen predoctoral y el seminario del Posgrado en Ingeniería Biomédica. 


\section{Maniobras respiratorias para la evaluación fisiológica.}

Se emplearon tres maniobras de respiración controlada de $150 \mathrm{~s}$ de duración, en dos de las cuales sólo cambiaba uno de los parámetros respiratorios mientras el otro se mantenía constante, y en la tercera cambiaban ambos de manera simultánea (Fig. 29). La primera maniobra independiente consistió en el incremento continuo y lineal de la FR ( $\left.\mathrm{FR}_{\mathrm{LI}}\right)$ desde 0.1 hasta $0.7 \mathrm{~Hz}$, manteniendo la amplitud del VC constante, a $0.8 \mathrm{~L}$ para las mujeres y $1 \mathrm{~L}$ para los hombres (Fig.33A). La segunda maniobra independiente consistió en mantener la FR constante a $0.2 \mathrm{~Hz}$ mientras el VC primero aumenta linealmente desde un mínimo de $0.25 \mathrm{~L}$ hasta un máximo $\left(\mathrm{VC}_{\mathrm{LI}}\right)$ y a continuación decrece desde el máximo hasta el mínimo $\left(\mathrm{VC}_{\mathrm{LD}}\right)$. Para los hombres, el máximo VC a alcanzar fue de $2.5 \mathrm{~L}$ y para las mujeres fue de $2.0 \mathrm{~L}$ (Fig. 29B). Finalmente, la maniobra combinada $\left(\mathrm{FR}_{\mathrm{LI}} / \mathrm{VC}_{\mathrm{LI}}-\mathrm{VC}_{\mathrm{LD}}\right)$ consistió en el incremento continuo de la $\mathrm{FR}$ superpuesto con la variación mixta del VC (Fig. 29C). Estas maniobras fueron realizadas en posición sentada.

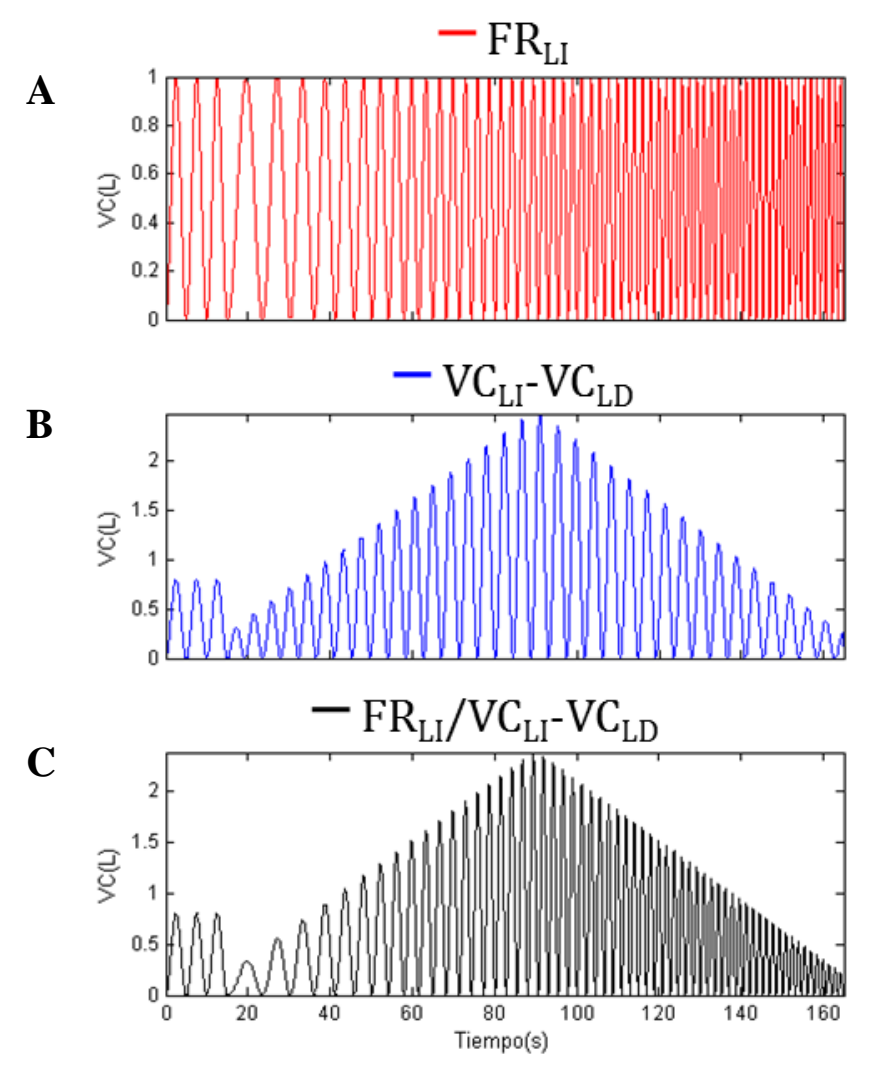

Fig.29. Patrones respiratorios para la evaluación fisiológica de los efectos de las variaciones lineales de las variables respiratorias, frecuencia respiratoria (FR) y volumen corriente (VC) sobre la ASR. FR incrementada a $\mathrm{VC}$ constante. $\mathrm{VC}_{\mathrm{LI}}-\mathrm{VC}_{\mathrm{LD}}$ : $\mathrm{VC}$ linealmente incrementado-linealmente reducido a FR constante. $\mathrm{FR}_{\mathrm{LI}} / \mathrm{VC}_{\mathrm{LI}}-\mathrm{VC}_{\mathrm{LD}}$ : variación simultánea de $\mathrm{FR}$ y $\mathrm{VC}$. 


\section{Maniobras para indicador vagal.}

Para poder sobreponer el control respiratorio con otras maniobras estimulantes del SNA, se desarrollaron tres maniobras de $30 \mathrm{~s}$ de duración, con una estructura similar a las maniobras fisiológicas pero con diferentes rangos de variación (Fig. 30). Así, la maniobra $\mathrm{FR}_{\mathrm{LI}}$ consistió en el incremento continuo y lineal de la FR desde 0.2 hasta $0.5 \mathrm{~Hz}$, a VC constante de $0.8 \mathrm{~L}$ para mujeres y $1 \mathrm{~L}$ para hombres; $\mathrm{VC}_{\mathrm{LI}^{-}}-\mathrm{VC}_{\mathrm{LD}}$, en un $\mathrm{VC}$ creciente y decreciente de 1 a $2.5 \mathrm{~L}$ para hombres y de 0.8 a $2.0 \mathrm{~L}$ para mujeres a FR constante de $0.2 \mathrm{~Hz}$; y $\mathrm{F}_{\mathrm{LI}^{-}} \mathrm{VC}_{\mathrm{LI}}-\mathrm{VC}_{\mathrm{LD}}$, en la variación simultánea de FR y VC. Para evaluar la capacidad de sobreponerse a otra maniobra estimulante del SNA, estas maniobras serán ejecutadas en posición sentada y durante un cambio de postura de sentado a parado, el cual se iniciará al finalizar la última respiración del periodo de control (Fig. 30).
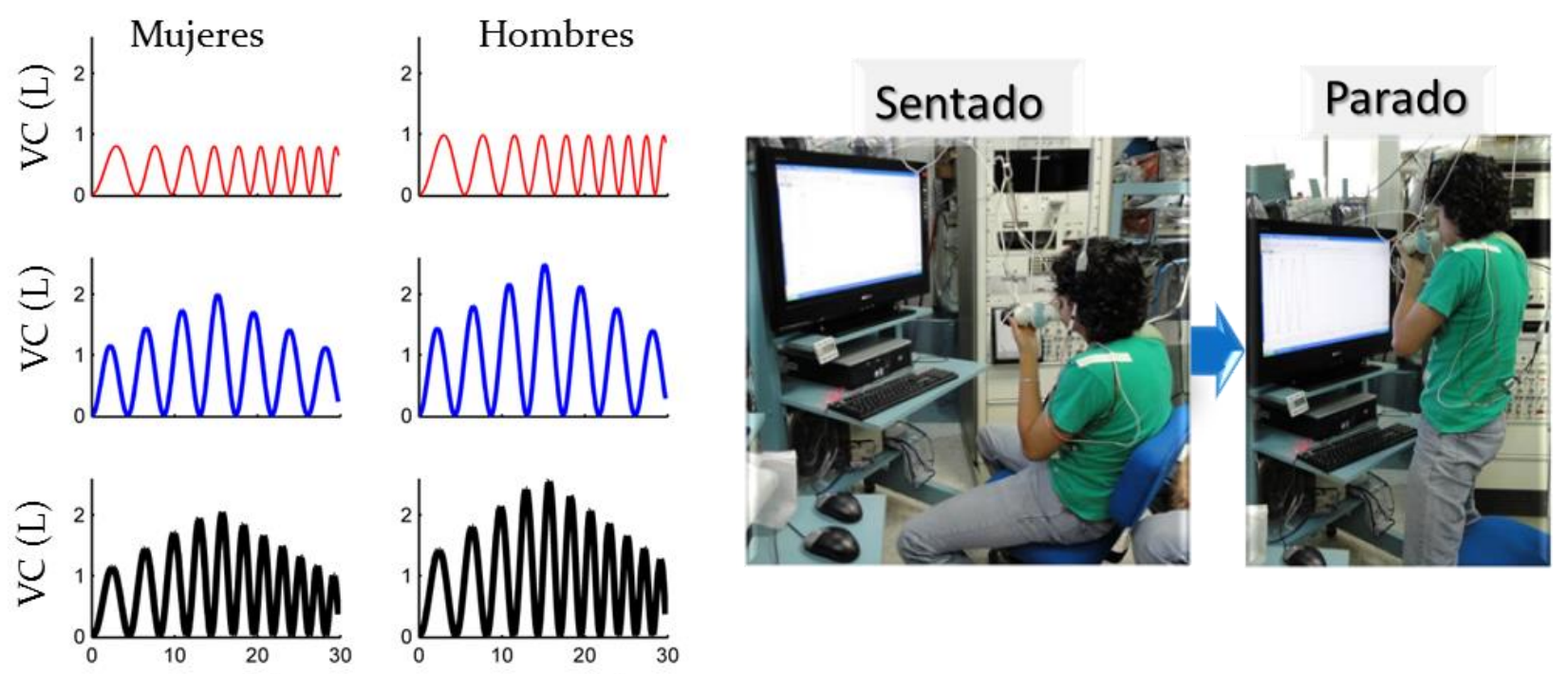

Tiempo (s)

Tiempo (s)

Fig.30. Maniobras respiratorias generadas para poder superponerse a otras maniobras fisiológicas, como el cambio activo de postura de sentado a parado. Presentan la misma estructura de las maniobras para la evaluación fisiológica, pero duran solamente 30 segundos y su rango de variación es menor.

\section{Registro de señales}

El ECG fue detectado en la derivación torácica bipolar CM-5 con un amplificador bioeléctrico (ECG100, Biopac Systems). Ninguno de los participantes presentó latidos ectópicos. El VC fue registrado mediante el conjunto formado por un neumotacómetro (HR100, Hans Rudolph), conectado a un transductor de presión (MP45, Validyne), un demodulador de acarreo (CD19A, Validyne) y un integrador (FV156, Validyne). De la altura de la boca se tomó continuamente una 
muestra de aire, cuya concentración de $\mathrm{CO}_{2}$ fue medida por un analizador infrarrojo (CO2100, Biopac Systems) calibrado con gases de concentración conocida. Todas las señales fueron digitalizadas a una frecuencia de muestreo de $500 \mathrm{~Hz}$ a través de un sistema de adquisición y despliegue (MP150, Biopac Systems) y almacenadas para su posterior procesamiento (Fig. 31).

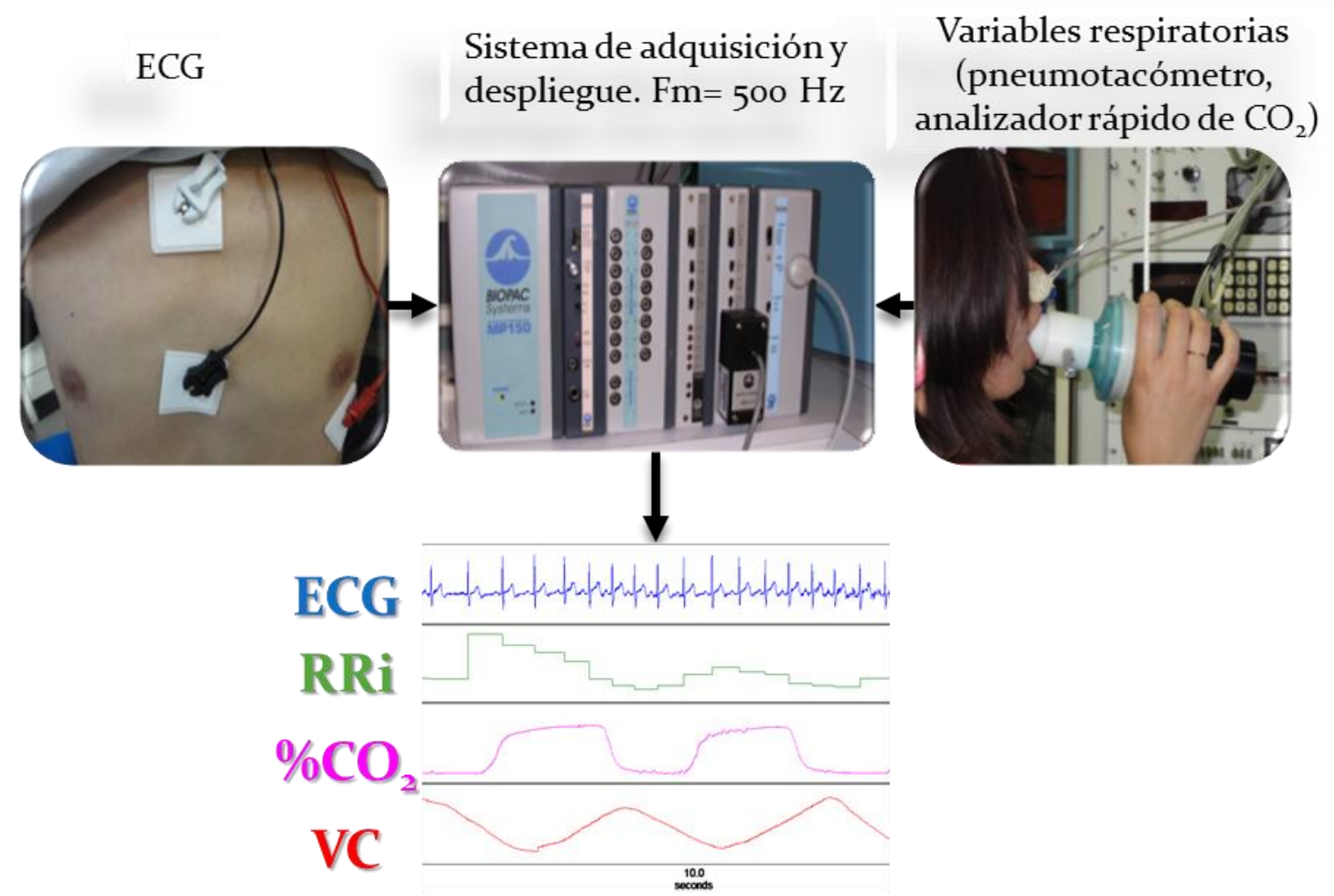

Fig.31. El ECG de los sujetos fue registrado a partir de la derivación torácica bipolar que minimiza los artefactos de movimiento y maximiza la onda R llamada CM5 (Task Force, 1996). Las variables respiratorias (VC y \%CO2) fueron registradas mediante un neumotacómetro conectado a una boquilla con tomas de aire. Todas las señales fueron adquiridas, desplegadas y almacenadas a una frecuencia de muestreo de $500 \mathrm{~Hz}$ por un sistema Biopac MP150.

\section{Procesamiento de datos}

El procesamiento fue efectuado fuera de línea utilizando programas escritos ex profeso en MatLab (R2012a, MathWorks).

El procesamiento consistió en los siguientes pasos (Fig. 32):

a. Detección de puntos característicos para la formación de series de tiempo: los máximos de las ondas R del ECG fueron detectados mediante la diferenciación estadística del complejo QRS (Gaitán et al. 1998) para formar la serie de intervalos RR o tacograma. Los valores máximos espiratorios de $\mathrm{CO}_{2}\left(\mathrm{ETCO}_{2}\right.$ por sus siglas en inglés, End-Tidal $\mathrm{CO}_{2}$ levels $)$ se calcularon 
como el valor máximo de cada ciclo del registro continuo del perfil de $\mathrm{CO}_{2}$. De la señal de volumen respiratorio fueron detectados los mínimos y máximos de cada ciclo para computar la FR y el VC en un formato respiración por respiración.

b. Preparación de las series de tiempo. El tacograma, respirograma y capnograma fueron interpolados y remuestreados empleando el método de splines cúbicos con una frecuencia de 8 Hz, a fin de obtener series de tiempo uniformemente muestreadas (Task Force 1996).

c. Procesamiento de la tendencia lineal. Las series de tiempo fueron divididas en nivel medio o tendencia y oscilaciones o variabilidad, utilizando el filtro smoothness priors con una frecuencia de corte de $0.04 \mathrm{~Hz}$ (Tarvainen et al. 2002). La tendencia de la serie RR obtenida por este método fue tomada como el $\mathrm{RR}_{\mathrm{m}}$.

d. Análisis espectral tiempo-frecuencia de las oscilaciones restantes del tacograma y el respirograma, que varían alrededor de cero, empleando la distribución pseudo Wigner-Villé suavizada, una representación de la clase de Cohen ampliamente utilizada en estudios fisiológicos (Cottin et al. 2004). Las ventanas de suavizado en tiempo y frecuencia fueron ajustadas de acuerdo a la longitud de las series de tiempo.

e. Estimación de las dinámicas de los índices espectrales y temporales. A partir de las distribuciones obtenidas del tacograma y respirograma se computaron como los primeros momentos de segundo orden las variaciones instantáneas en la potencia de sus componentes de AF en unidades absolutas y sus frecuencias centrales en la banda de 0.15 a $0.7 \mathrm{~Hz}$ (Monti et al. 2002). La VP fue calculada como producto entre el VC y la FR instantánea.

f. Análisis de las dinámicas. Mediante integración numérica trapezoidal se obtuvo el área bajo la curva de todas las dinámicas respiratorias y cardiovasculares durante las etapas de control y maniobra, así como la diferencia control - maniobra como índice de la magnitud del cambio inducido. 

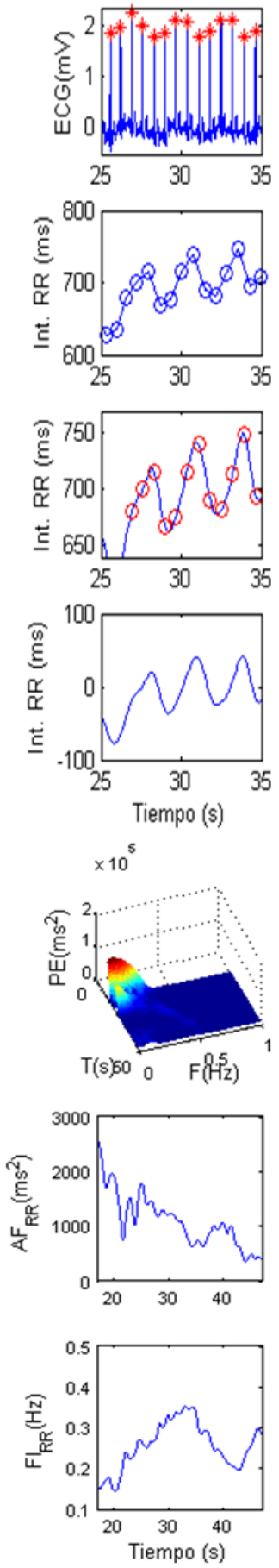

Detección de puntos importantes

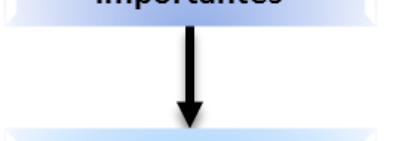

Formación de series de tiempo

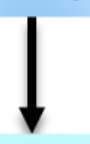

Interpolación y remuestreo a $8 \mathrm{~Hz}$

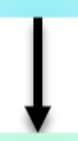

\section{Remoción de} tendencia

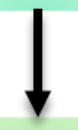

Cálculo DTF PWVS

\section{Estimación de}

parámetros espectrales, banda FR

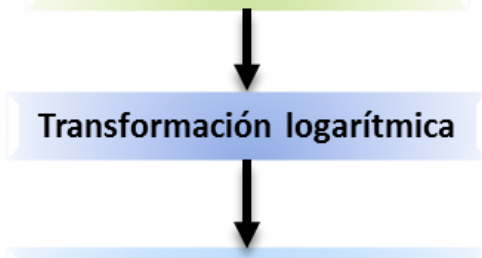

Formación de relaciones entre variables respiratorias y $\ln A F_{R R}$

Análisis estadístico: Regresión y correlación lineales

Prueba de T pareada
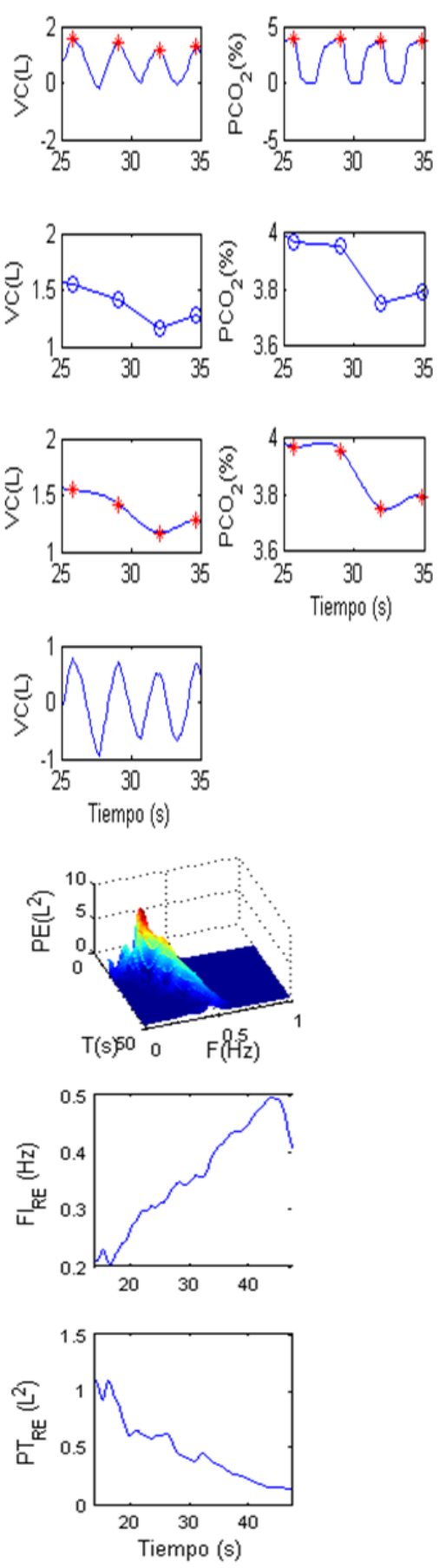

Fig.32. Etapas del procesamiento fuera de línea. Brevemente, a partir de la detección de puntos fiduciarios del ECG (máximo de la onda R), el VC y el \% $\mathrm{CO}_{2}$, se formaron series de tiempo, que fueron interpoladas y remuestreadas a $8 \mathrm{~Hz}$ por splines cúbicos y se les extrajo la tendencia por el método Smoothness priors para poder obtener su distribución tiempo-frecuencia mediante la transformación pseudo-Wigner-Villé suavizada. La dinámica de la potencia de los componentes espectrales fue estimada en la banda de la FR para formar relaciones entre variables respiratorias y autonómico-cardiovasculares. 


\section{Análisis estadístico}

Los datos se expresan como media \pm desviación estándar. La normalidad de los datos fue evaluada con la prueba de Kolmogórov-Smirnov. Debido a que presentó una distribución sesgada, el componente de $\mathrm{AF}_{\mathrm{RR}}$ fue normalizado mediante una transformación logarítmica (ln $\left.\mathrm{AF} \mathrm{F}_{\mathrm{RR}}\right)$.

Con la finalidad de caracterizar el efecto de las variables respiratorias sobre la respuesta cardiovascular se construyeron las relaciones entre $\ln \mathrm{AF}_{\mathrm{RR}}$ (o su frecuencia instantánea, FIAF $_{\mathrm{RR}}$ ) y el VC o la FR. El grado de asociación lineal entre las variables estudiadas fue evaluado mediante el ajuste de regresiones lineales simples y el cómputo del coeficiente de correlación de las relaciones $\ln \mathrm{AF}_{\mathrm{RR}}-\mathrm{FR}$ y $\ln \mathrm{AF}_{\mathrm{RR}}-\mathrm{VC}_{\mathrm{LI}}-\mathrm{VC}_{\mathrm{LD}}$ de cada sujeto. Para facilitar su caracterización matemática y el análisis estadístico, las respuestas de $\ln \mathrm{AF}_{\mathrm{RR}}$ a las maniobras con $\mathrm{VC}_{\mathrm{LI}}-\mathrm{VC}_{\mathrm{LD}}$ fueron divididas en las partes creciente y decreciente. La diferencia entre los interceptos $(\Delta \mathrm{I}) \mathrm{de}$ las regresiones $\ln \mathrm{AF}_{\mathrm{RR}}-\mathrm{VC}_{\mathrm{LI}}$ y $\ln \mathrm{AF}_{\mathrm{RR}}-\mathrm{VC}_{\mathrm{LD}}$ se tomó como una medida de la histéresis.

La integral de las dinámicas instantáneas de $\mathrm{VP}, \mathrm{RR}_{\mathrm{m}}$ y $\ln \mathrm{AF}_{\mathrm{RR}}$ se utilizó para calcular sus valores medios durante el periodo de control y durante la maniobra. Para el $\mathrm{ETCO}_{2}$, se consideraron el promedio del control y el valor al final de la maniobra. A fin de obtener una medida del efecto global de la maniobra, se obtuvieron las diferencias control-maniobra de los valores medios.

Para mejorar la visualización de los efectos, se obtuvieron los promedios ensamblados de las dinámicas individuales de las variables y sus relaciones.

La prueba t de Student para muestras pareadas fue empleada para comparar los parámetros de las regresiones lineales de las maniobras independientes y las combinadas. Las diferencias entre géneros fueron analizadas mediante una prueba de $\mathrm{t}$ para muestras independientes. Las comparaciones entre los valores medios durante el control, maniobra y la diferencia controlmaniobra fueron efectuadas mediante ANOVA para mediciones repetidas con comparaciones post-hoc por la prueba de Tukey. El nivel de significancia aceptado fue $\mathrm{p}<0.05$. 


\section{Resultados}

\section{Protocolos para fisiología}

En la Fig. 33 se muestran los patrones respiratorios generados para el examen fisiológico de la variación simultánea de la FR y el VC. A la derecha de los patrones respiratorios están representados los promedios ensamblados de las relaciones VC-FR producidas por los sujetos al ejecutar cada maniobra, y muestran que las tres maniobras fueron correctamente realizadas. La relación VC-FR correspondiente al patrón doblemente variante $\mathrm{FR}_{\mathrm{LI}} / \mathrm{VC}_{\mathrm{LI}}-\mathrm{VC}_{\mathrm{LD}}$ presentó una correlación cercana a cero y no significativa $(r=0.01 \pm 0.01, p>0.05)$. El error instantáneo, calculado como la resta punto a punto de cada patrón respiratorio y la señal respiratoria correspondiente, fue menor al $10 \%$ del valor máximo del patrón, indicando que los sujetos fueron capaces de seguir fielmente al patrón respiratorio.
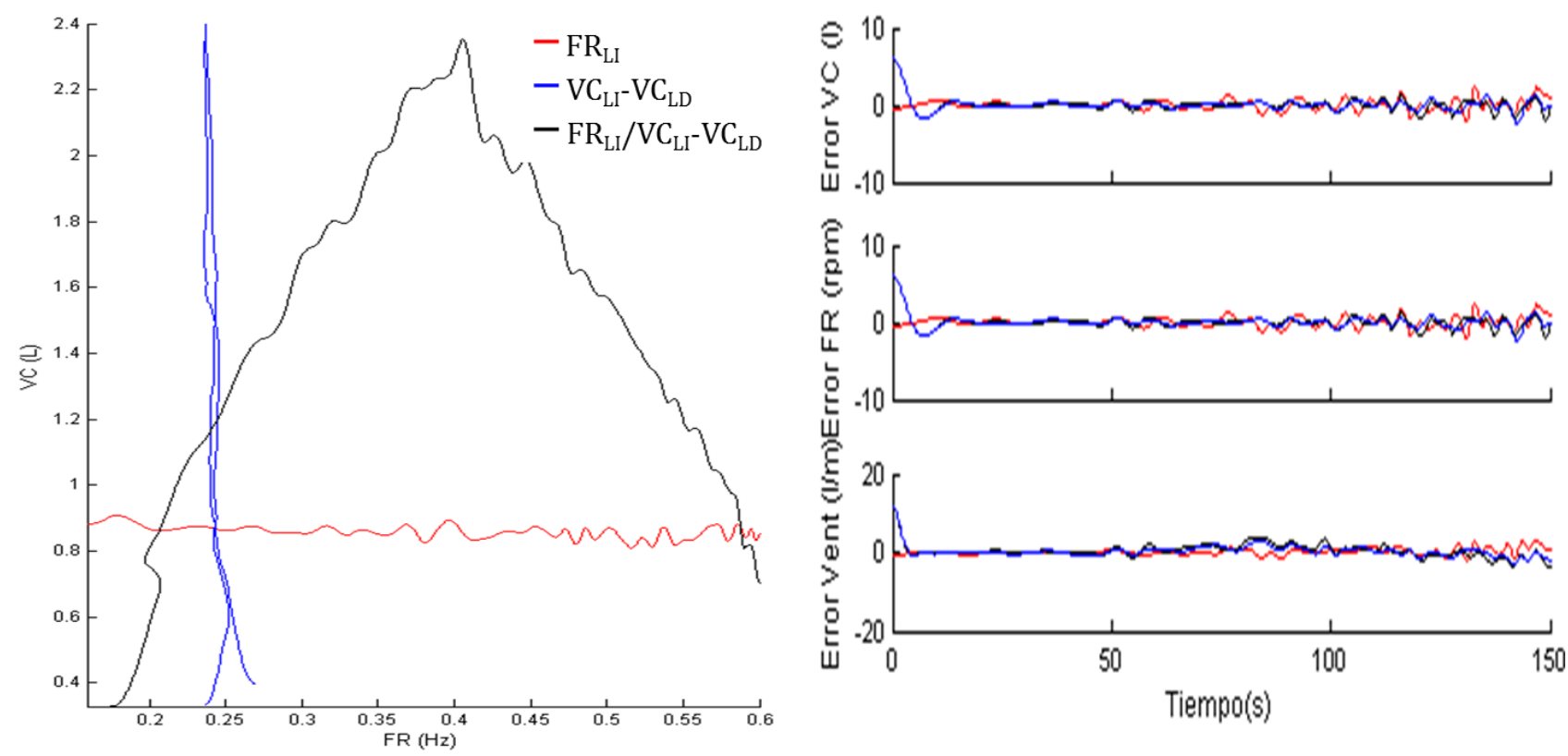

Fig.33. Evaluación del desempeño de los sujetos al seguir las maniobras respiratorias para la evaluación fisiológica. A) gráfica de VC vs. FR, donde se aprecian los diferentes patrones de variación de las variables respiratorias propios de cada maniobra. B) Errores instantáneos del VC (fila superior), la FR (fila media) y el producto VCxFR o ventilación pulmonar (fila inferior) durante las tres maniobras.

En la Fig. 34 se muestra el comportamiento en el tiempo de las variables estímulo (VC y FR) así como de las variables respuesta $\left(\operatorname{lnAF}_{\mathrm{RR}}, \mathrm{RR}_{\mathrm{m}} \mathrm{y} \mathrm{ETCO}_{2}\right)$ a los tres protocolos empleados. El componente de $\mathrm{AF}_{\mathrm{RR}}$ fue no lineal en las maniobras donde el VC cambió. 
Se observaron dos tipos de respuesta: a baja FR (menor a $0.3 \mathrm{~Hz}$ ) y bajo VC (menor a $1.5 \mathrm{~L}$ ) la magnitud del efecto del protocolo doblemente variante sobre el $\ln A F_{R R}$ fue ligeramente mayor que el efecto de los protocolos univariantes; pero por encima de estos valores, a FR y VC mayores, se observó una disminución progresiva no lineal.

La comparación de los valores de $\ln \mathrm{AF}_{\mathrm{RR}}$ alcanzados durante cada maniobra en el punto donde la respuesta a la maniobra $\mathrm{FR}_{\mathrm{L}} / \mathrm{VC}_{\mathrm{LI}}-\mathrm{VC}_{\mathrm{LD}}$ alcanzó el mínimo evidenció la mayor disminución

$$
-\mathrm{FR}_{\mathrm{LI}}-\mathrm{VC}_{\mathrm{LI}}-\mathrm{VC}_{\mathrm{LD}}-\mathrm{FR}_{\mathrm{LI}} / \mathrm{VC}_{\mathrm{LI}}-\mathrm{VC}_{\mathrm{LD}}
$$

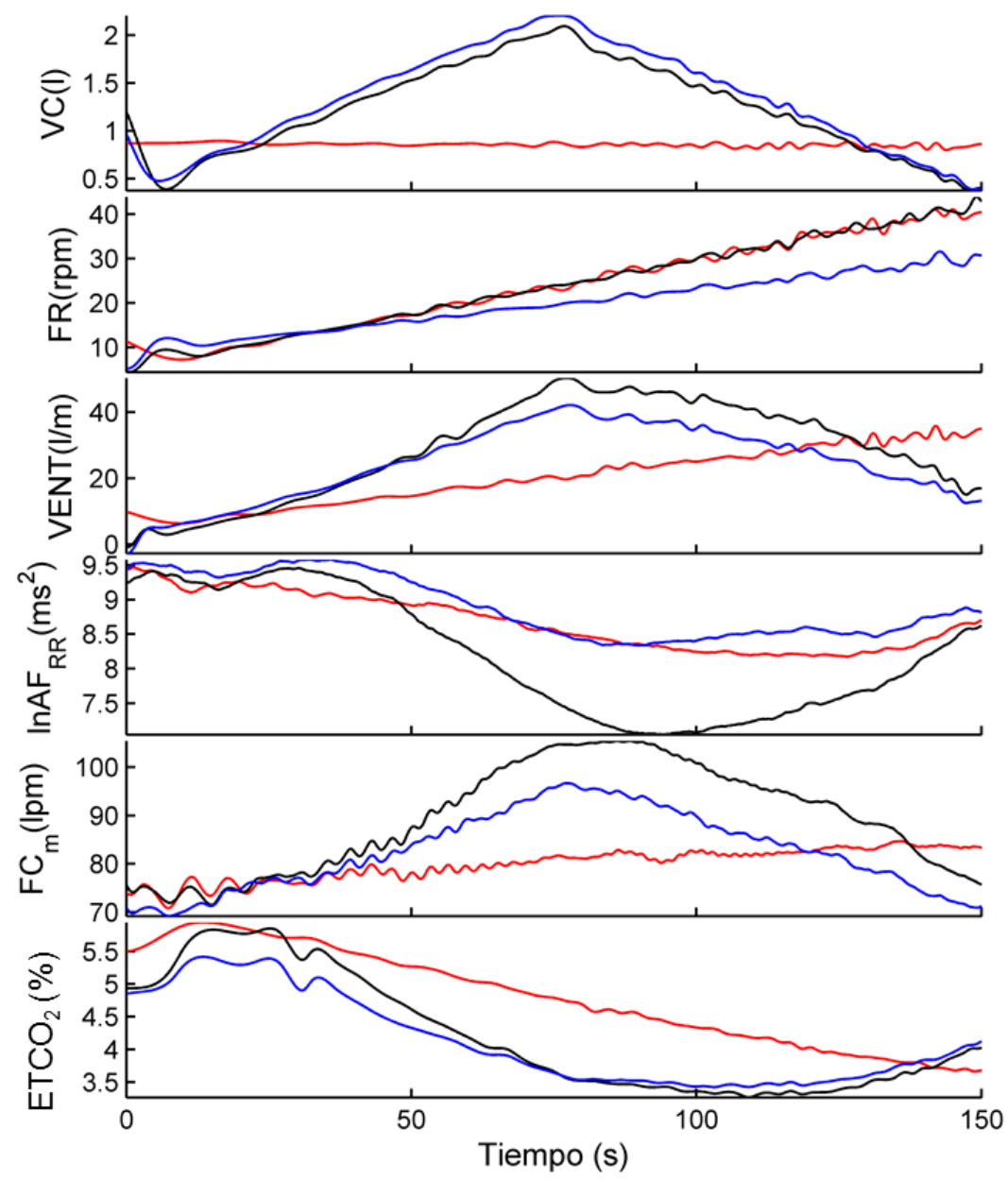

Fig.34. Dinámicas de las variables respiratorias y cardiovasculares durante la realización de los protocolos para la evaluación fisiológica de los efectos de la variación continua y lineal de la FR y el VC aplicadas de manera independiente y simultánea. Cada línea corresponde al promedio ensamblado de las dinámicas de todos los sujetos estudiados. provocada por la maniobra simultánea $\quad(\mathrm{p}<0.05) \quad$ en relación a la producida por la maniobra $\mathrm{FR}_{\mathrm{LI}}$

(FR incremental, VC constante), la cual a su vez fue mayor que la causada por el protocolo $\mathrm{VC}_{\mathrm{LI}^{-}}$ $\mathrm{VC}_{\mathrm{LD}}$. Estos resultados sugieren que ocurre un efecto sinérgico cuando ambos estímulos son aplicados simultáneamente.

El incremento en la frecuencia cardiaca media (FCm) causado por el protocolo doblemente variante fue significativamente mayor $(\mathrm{p}<0.05)$ respecto a las otras maniobras (Fig. 34), lo que sugiere que las dos variables ventilatorias también produjeron sinergia en el tono vagal.

La dinámica del $\mathrm{ETCO}_{2}$ en respuesta a las tres maniobras consistió en la transición progresiva de hiper $\left(\mathrm{FR}_{\mathrm{L}} / \mathrm{VC}_{\mathrm{LI}}-\mathrm{VC}_{\mathrm{LD}} \mathrm{y}\right.$ $\mathrm{FR}_{\mathrm{LI}}$ ) o normocapnia $\left(\mathrm{VC}_{\mathrm{LI}}-\mathrm{VC}_{\mathrm{LD}}\right)$ a hipocapnia (Fig. 38). A bajos $\mathrm{FR}$ y VC, la hipercapnia del 
protocolo $\mathrm{FR}_{\mathrm{LI}} / \mathrm{VC}_{\mathrm{LI}}-\mathrm{VC}_{\mathrm{LD}}$ fue equivalente a la del protocolo $\mathrm{FR}_{\mathrm{LI}}$, pero a $\mathrm{FR}$ mayores a $0.3 \mathrm{~Hz}$ la disminución producida por la maniobra doblemente variante fue más pronunciada $(\mathrm{p}<0.01)$ que las correspondientes a las otras maniobras. Por tanto, las tres maniobras causaron hipocapnia, cuya contribución a la respuesta del componente de $\mathrm{AF}_{\mathrm{RR}}$ debe ser discutida.

La Fig. 35 contiene la representación tridimensional del promedio ensamblado de la relación $\ln A F_{R R}-F R-V C$ y sus proyecciones sobre los planos $\ln A F_{R R}-V C$ y $\ln A F_{R R}-F R$. Superpuestas a estas últimas se encuentran los promedios ensamblados de las relaciones $\ln \mathrm{AF}_{\mathrm{RR}}-\mathrm{VC}_{\mathrm{y}} \ln \mathrm{AF}_{\mathrm{RR}}-\mathrm{FR}$ obtenidas durante las maniobras $\mathrm{VC}_{\mathrm{LI}}-\mathrm{VC}_{\mathrm{LD}}$ y $\mathrm{FR}_{\mathrm{LI}}$ respectivamente.

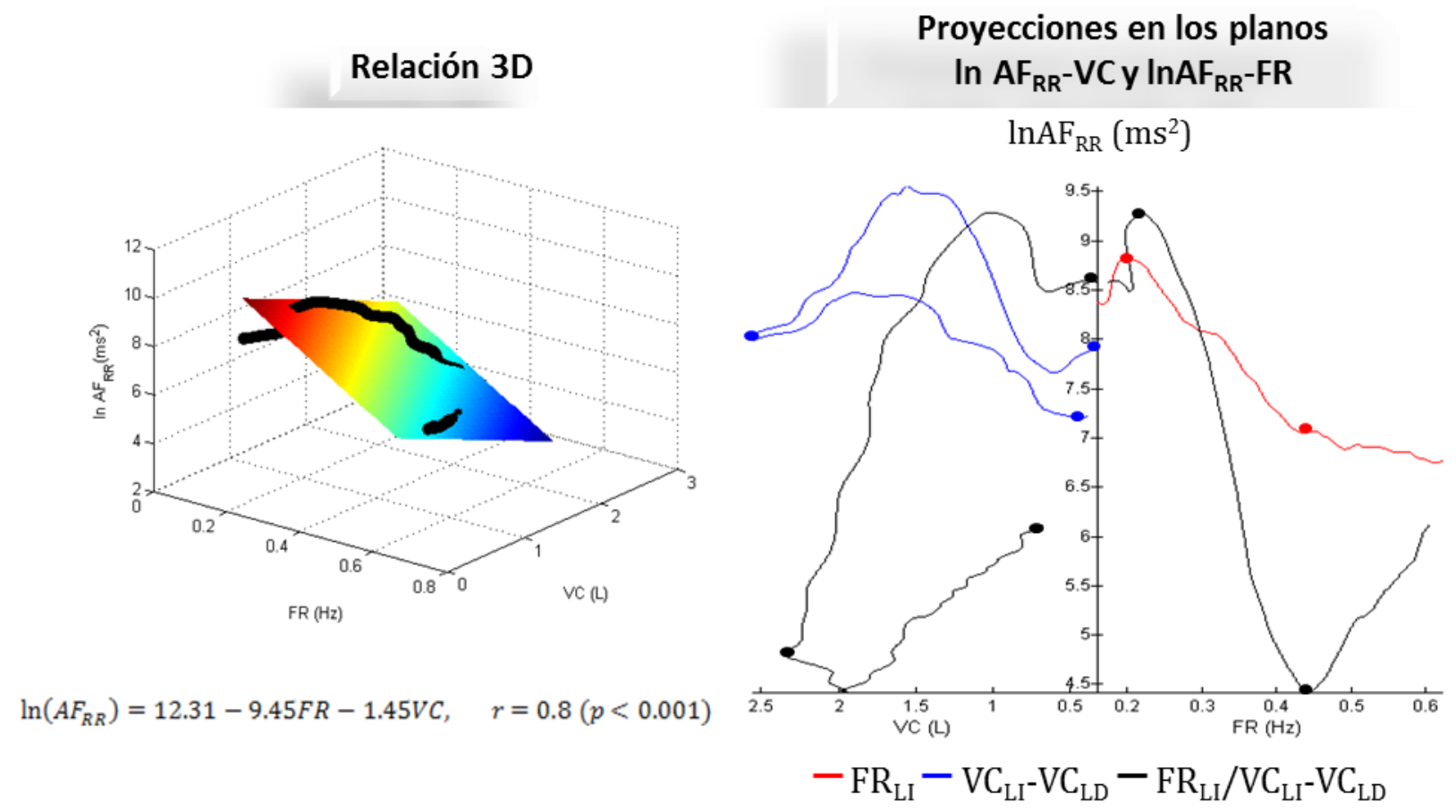

Fig.35. La variación simultánea de las variables ventilatorias produce un efecto aditivo sobre el sistema núcleos vagales-nodo $S A$, ocasionando una mayor disminución de $\operatorname{InAF}_{\mathrm{RR}}$, en comparación a la obtenida durante la variación independiente de FR y VC.

Con excepción de la relación $\ln \mathrm{AF}_{\mathrm{RR}}-\mathrm{FR}$ en respuesta al protocolo $\mathrm{FR}_{\mathrm{LI}}$, el resto de las relaciones son no lineales $(\mathrm{p}<0.001)$. En las proyecciones de la respuesta sobre los planos $\ln \mathrm{AF}_{\mathrm{RR}}-\mathrm{FR}$ y $\ln \mathrm{AF}_{\mathrm{RR}}-\mathrm{VC}$ se corroboraron los resultados observados en el tiempo: a FR menores a $0.3 \mathrm{~Hz}$ y VC menores a $1.25 \mathrm{~L}$, el $\ln \mathrm{AF}_{\mathrm{RR}}$ en respuesta a la maniobra doblemente variante fue mayor $(\mathrm{p}<0.001)$ a las respuestas de las otras dos maniobras; pero, por encima de estos valores, la disminución del $\ln \mathrm{AF}_{\mathrm{RR}}$ producida por el protocolo $\mathrm{FR}_{\mathrm{LI}} / \mathrm{VC}_{\mathrm{LI}}-\mathrm{VC}_{\mathrm{LD}}$ fue mucho mayor $(\mathrm{p}<0.001)$ que la causada por los otros protocolos. Estos resultados documentan que las variaciones simultáneas del VC y la FR producen un efecto autonómico cardiovascular mayor que el causado por la modificación 
voluntaria de una de las variables mientras se mantiene constante la otra (Fig. 35). Esta respuesta soporta la noción de que el sistema formado por los núcleos vagales y el nodo sinusal integra ambas entradas, produciendo un efecto mayor. Este hallazgo se contrapone a la noción tradicionalmente aceptada de que a mayor VC corresponde mayor ASR. Hasta donde sabemos, este hallazgo no ha sido publicado previamente. Una influencia que puede contribuir al efecto sobre el $\ln \mathrm{AF}_{\mathrm{RR}}$ es la hipocapnia producida por la maniobra simultánea.

En la Fig. 36 se ilustra el efecto de tres protocolos con variaciones lineales de VC, todos a FR constante, sobre $\ln \mathrm{AF}_{\mathrm{RR}}$. Sobresale que: a) las tres respuestas fueron no lineales; b) la respuesta al protocolo creciente no fue superponible a la del creciente, existe histéresis; c) la respuesta al protocolo creciente-decreciente fue aproximadamente superponible a la de los otros protocolos; d) entre 0.5 y $1.4 \mathrm{~L}$ la respuesta fue proporcionalmente creciente, por lo que consideramos a este último VC como un valor umbral, más allá del cual la respuesta ya no cambia o disminuye. Los anteriores hallazgos soportan la noción de una cierta persistencia de los efectos del VC sobre la actividad de los núcleos vagales - nodo SA: la excitabilidad actual depende de la historia previa, o quizá la hipoxia podría tener efectos en la sensibilidad del SNA a cambios en el VC. Además, sugieren que existe un límite de VC por encima del cual la respuesta del sistema ya no aumenta: existe un límite en la respuesta a cambios al VC. Estos hallazgos retan al conocimiento aceptado de una proporcionalidad entre la ASR y el VC.

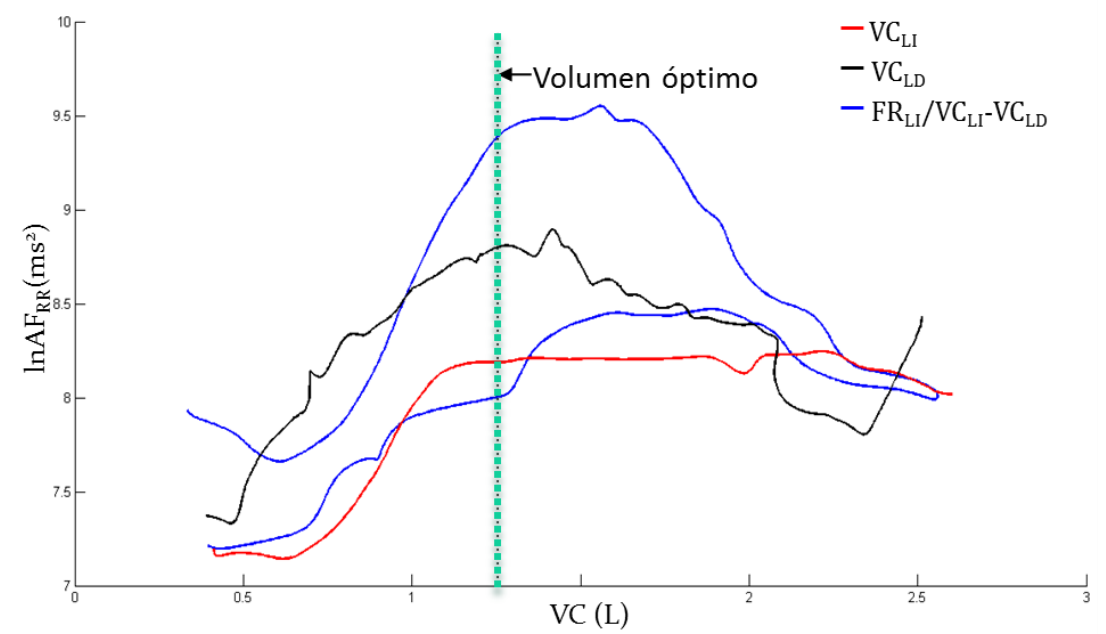

Fig.36. Caracterización del efecto del VC sobre el InAF $F_{R R}$. Las relaciones $\operatorname{InAF_{RR}-VC}$ obtenidas durante la ejecución de los tres protocolos donde la FR se mantuvo constante y el VC cambió de manera linealmente creciente, decreciente 0 crecientedecreciente fueron no lineales y presentaron histéresis. Alrededor de los $1.4 \quad \mathrm{~L}$ presentaron un valor óptimo, después del cual el $\operatorname{InAF}_{\mathrm{RR}}$ ya no cambia o disminuye aunque el VC siga aumentando.

Por definición, el fenómeno de la ASR presupone la existencia de coherencia entre la FR y la $\mathrm{FIAF}_{\mathrm{RR}}$. Proponemos la construcción de la relación $\mathrm{FIAF}_{\mathrm{RR}}-\mathrm{FR}$ para evaluar este fenómeno. Cuando exista coherencia entre ambas frecuencias, esta relación se sobrepondrá o estará cerca de 
la línea de identidad. En la Fig. 37 se encuentran graficados los promedios ensamblados de las relaciones $\mathrm{FIAF}_{\mathrm{RR}}-\mathrm{FR}$ obtenidas para los protocolos donde la $\mathrm{FR}$ cambiaba $\left(\mathrm{FR}_{\mathrm{LI}}\right.$ y $\mathrm{FR}_{\mathrm{LI}} / \mathrm{VC}_{\mathrm{LI}^{-}}$ $\mathrm{VC}_{\mathrm{LD}}$ ), junto con el comportamiento esperado (línea punteada). Las relaciones FIAF $\mathrm{RR}-\mathrm{FR}$ de ambos protocolos se encuentran por debajo de la línea de identidad, aunque la separación se acentúa a partir de $0.3 \mathrm{~Hz}$ y sigue aumentando conforme la FR se incrementa. Más aún, esta divergencia es mayor para el protocolo $\mathrm{FR}_{\mathrm{LI}} / \mathrm{VC}_{\mathrm{LI}}-\mathrm{VC}_{\mathrm{LD}}$.

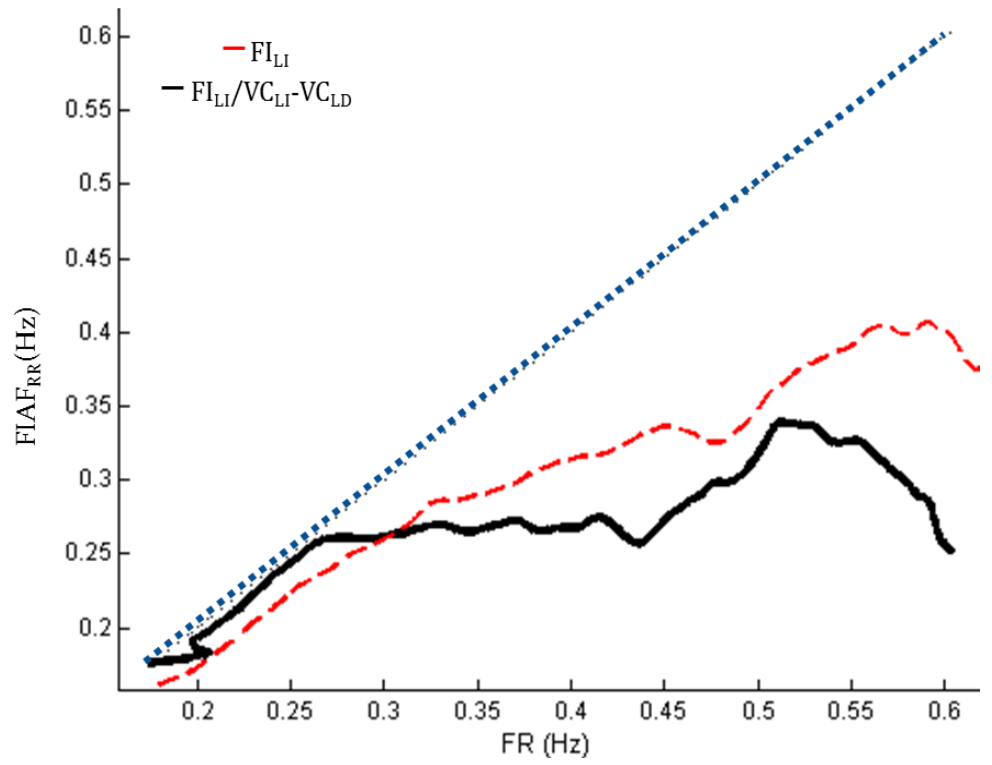

Fig. 37. Promedios ensamblados de la relación propuesta para evaluar la coherencia entre la frecuencia respiratoria (FR) y la frecuencia central instantánea del componente de $\mathrm{AF}_{\mathrm{RR}}$ $\left(\mathrm{FIAF}_{\mathrm{RR}}\right)$. Se observa una separación del comportamiento esperado a FR menores a $0.3 \mathrm{~Hz}$.

\section{Protocolos para indicador vagal}

Los protocolos de duración reducida y rangos de FR y VC ajustados a los valores de ejecución más cómoda se muestran en la Fig. 38, acompañados de los promedios ensamblados de las relaciones VC-FR correspondientes a cada maniobra en las dos posturas. Los patrones obtenidos respaldan que las maniobras respiratorias de los tres protocolos fueron adecuadamente ejecutadas por los sujetos. El coeficiente de correlación de la relación VC-FR fue de $0.1 \pm 0.04$, p >0.05 en el caso de la maniobra doblemente variante $\mathrm{FR}_{\mathrm{LI}} / \mathrm{VC}_{\mathrm{LI}}-\mathrm{VC}_{\mathrm{LD}}$. 

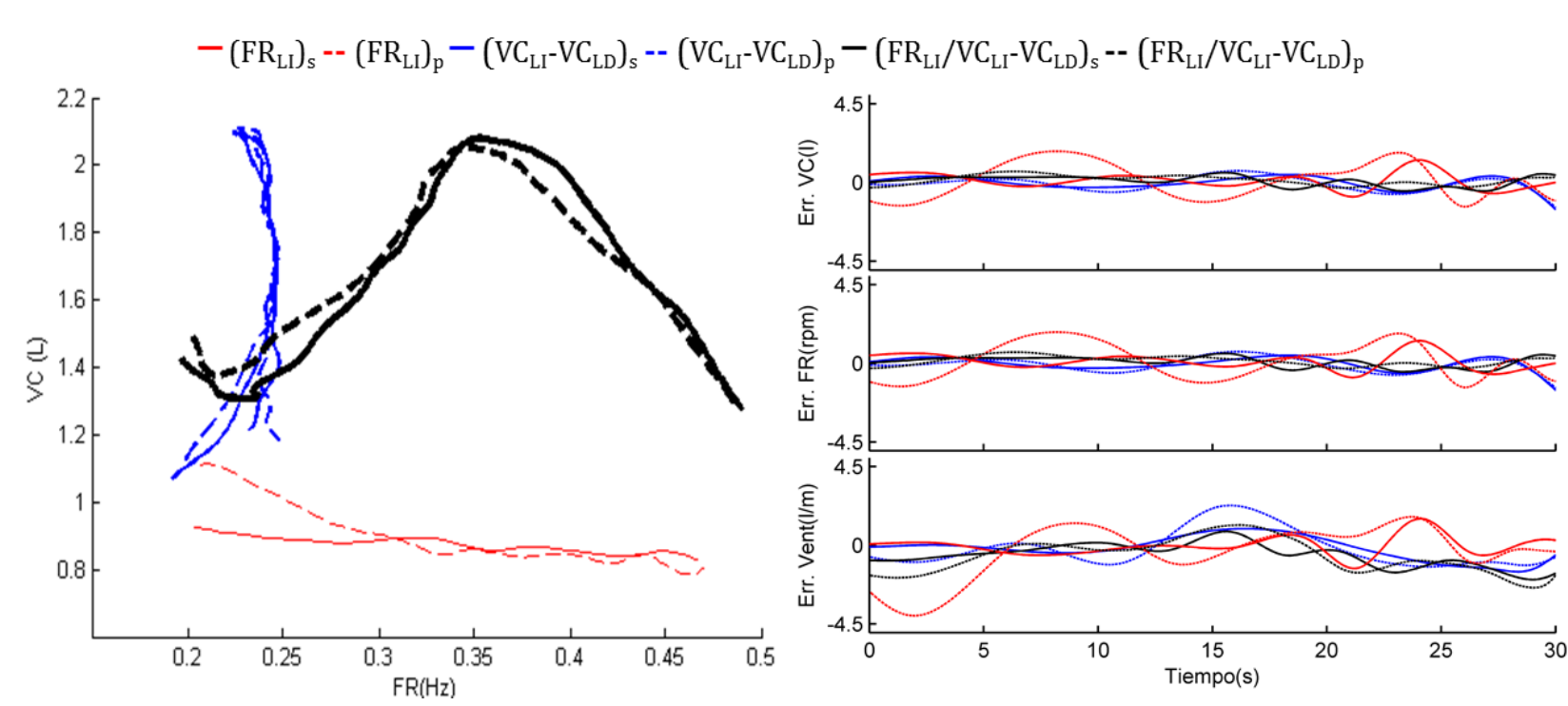

Fig. 38. Evaluación del desempeño de los sujetos al seguir las maniobras respiratorias para la superposición de otras maniobras, en este caso, el cambio de postura activo de sentado a parado. A) gráfica VC vs. FR, donde se aprecian los diferentes patrones de variación de las variables respiratorias propios de cada maniobra. B) Errores instantáneos del VC (fila superior), la FR (fila media) y el producto VCxFR o ventilación minuto (fila inferior) durante las tres maniobras.

El comportamiento de las variables estímulo y las variables respuesta a los tres protocolos se muestra en la Fig. 39. El comportamiento de los patrones ensamblados de la respuesta autonómica durante las maniobras de corta duración concuerda y corrobora lo observado durante las maniobras de evaluación fisiológica: el $\ln \mathrm{AF}_{\mathrm{RR}}$ disminuyó más durante la maniobra simultánea que durante las maniobras univariantes en la mayor parte del rango estudiado, tanto en posición sentada como parada.

Una diferencia notable respecto a la respuesta durante los protocolos para estudio fisiológico es que el componente de $\mathrm{AF}_{\mathrm{RR}}$ presentó una disminución lineal con el tiempo, como documentada por la prueba estadística de linealidad y el alto coeficiente de correlación, durante los tres protocolos en la posición sentada. El coeficiente de correlación disminuyó en la posición erguida reflejando el cambio de presión arterial oscilatorio producido por el cambio de postura como puede observarse en la Fig. 39.

Puesto que la mayoría de las relaciones cambió de manera lineal fue posible caracterizar el efecto del cambio de postura por la variación en la pendiente y el intercepto. Si se toma como referencia la respuesta durante sentado, el efecto de ejecutar las maniobras respiratorias en la posición erguida fue siempre provocar reducciones en los parámetros la relación respectiva. 


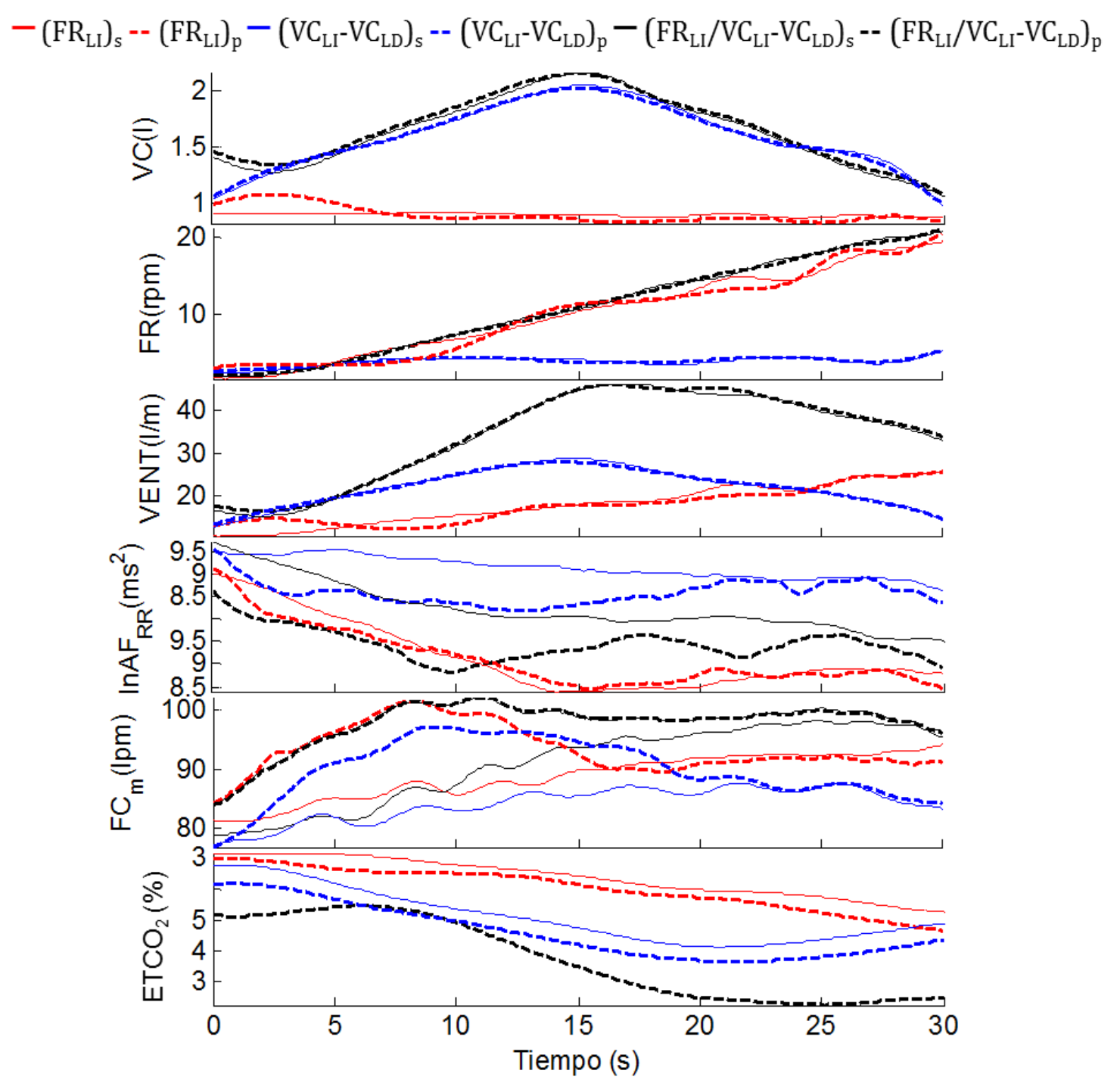

Fig. 39. Dinámicas de las variables respiratorias y cardiovasculares durante la realización de las maniobras de respiración controlada superpuesta con el cambio de postura activo de sentado a parado. Las líneas continuas corresponden a la postura sentada y las líneas punteadas a la postura erguida. Es importante notar que el control respiratorio no presentó diferencias entre una postura y otra, mientras que las variables indicadoras de la función autonómica-cardiovascular sí presentaron diferencias: en la postura erguida hubo mayor $\mathrm{FC}_{\mathrm{m}}$ y menor $\mathrm{nnAF}_{\mathrm{RR}}$, además de que se presentaron dinámicas más complejas que las observadas en estas mismas maniobras respiratorias realizadas en la postura sentada. El hecho de que las variables respiratorias no mostraron diferencias garantiza que estos cambios en los indicadores del control autonómico-cardiovascular se deben únicamente al cambio de postura.

En el panel izquierdo de la Fig. 40 se encuentra la representación tridimensional de la respuesta del $\ln \mathrm{AF}_{\mathrm{RR}}$ a la variación simultánea de la FR y el VC en las dos posturas, y en el panel derecho, las proyecciones de esta relación en la gráfica tipo Margaria, superpuestas a las relaciones $\ln \mathrm{AF}_{\mathrm{RR}}$ $-\mathrm{VC}$ y $\ln \mathrm{AF}_{\mathrm{RR}}-\mathrm{FR}$ obtenidas durante las maniobras $\mathrm{VC}_{\mathrm{LI}}-\mathrm{VC}_{\mathrm{LD}}$ y $\mathrm{FR}_{\mathrm{LI}}$ respectivamente. Con excepción de las relaciones respecto al VC, tanto la relación tridimensional como la proyección sobre el plano $\ln \mathrm{AF}_{\mathrm{RR}}-\mathrm{FR}$ cumplieron con la hipótesis de linealidad en las dos posturas $(\mathrm{p}<0.001)$ 
y tuvieron altos coeficientes de correlación $(r s=0.9 \pm p<0.001, r p=0.8 \pm p<0.001)$. La sobreposición de la entrada barorrefleja modificó la respuesta autonómica como un todo: el plano completo de la correlación múltiple, así como los parámetros de las relaciones en respuesta a las maniobras univariante se redujeron, indicio claro de la ocurrencia de la inhibición del sistema núcleos vagales-nodo sinusal causada por el cambio de postura.

El cambio de postura tuvo el efecto de disminuir significativamente $(\mathrm{p}<0.001)$ los coeficientes de la regresión múltiple correspondientes a la FR y al intercepto.
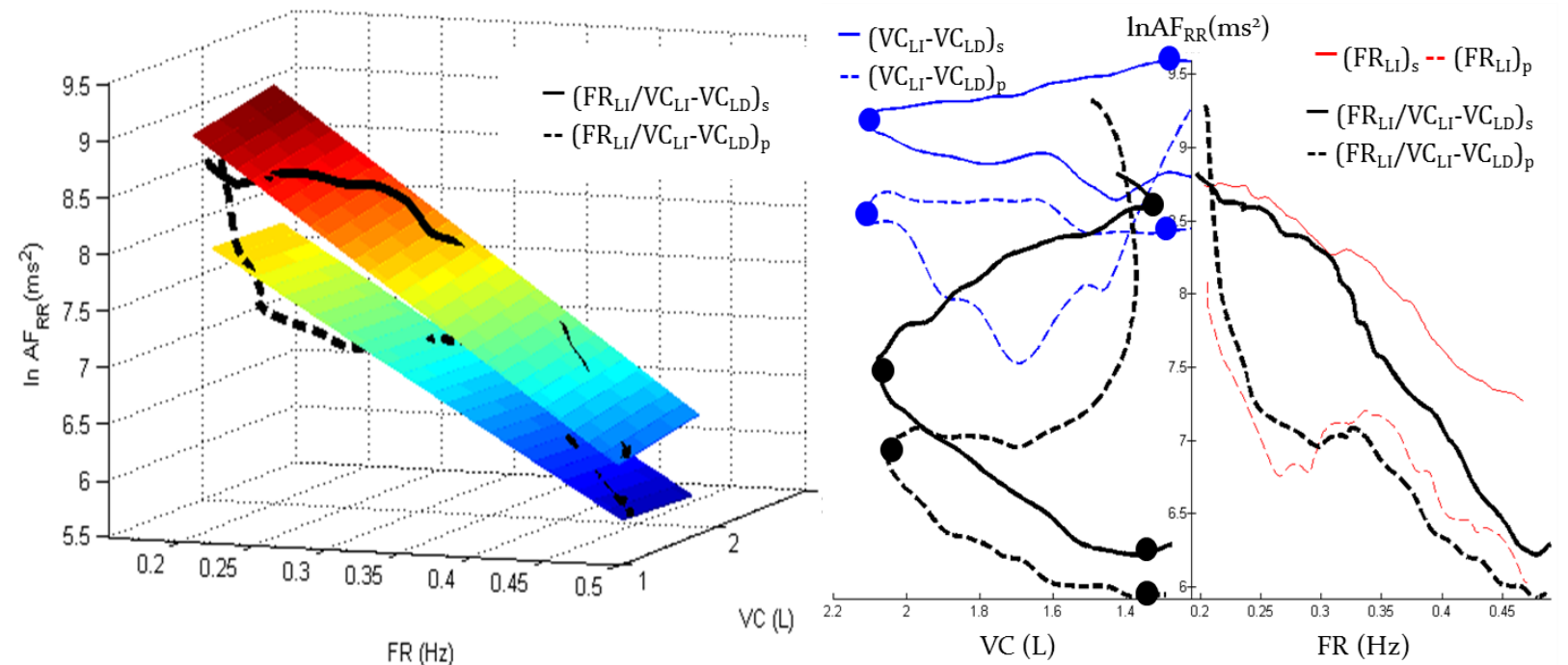

Fig. 40. La superposición del cambio de postura ocasionó una mayor disminución de la potencia de $\mathrm{AF}_{\mathrm{RR}}$ en todas las maniobras, tanto independientes como combinadas. También aparecieron oscilaciones que redujeron los coeficientes de correlación tanto de las regresiones simples como de las múltiples. Puesto que la respiración fue igual en ambas posturas, podemos atribuir inequívocamente estos cambios en el comportamiento del indicador vagal a la respuesta autonómico-cardiovascular al cambio de postura.

$$
\begin{gathered}
\ln \left(A F_{R R}\right) S=10.9-10.5 F R+0.2 V C, r=0.9(p<0.001) \\
\ln \left(A F_{R R}\right) P=8.9-7.6 F R+0.3 V C, r=0.8(p<0.001)
\end{gathered}
$$

\section{Evaluación de diferencias entre sexos}

En la Fig. 41 se muestran los promedios ensamblados de las señales respiratorias de hombres y mujeres, superpuestos al patrón respiratorio que siguieron durante las maniobras FR $_{\mathrm{LI}}$ (Fig. 41A), $\mathrm{VC}_{\mathrm{LI}}-\mathrm{VC}_{\mathrm{LD}}$ (Fig. 41B) y la maniobra combinada (Fig. 41C). 

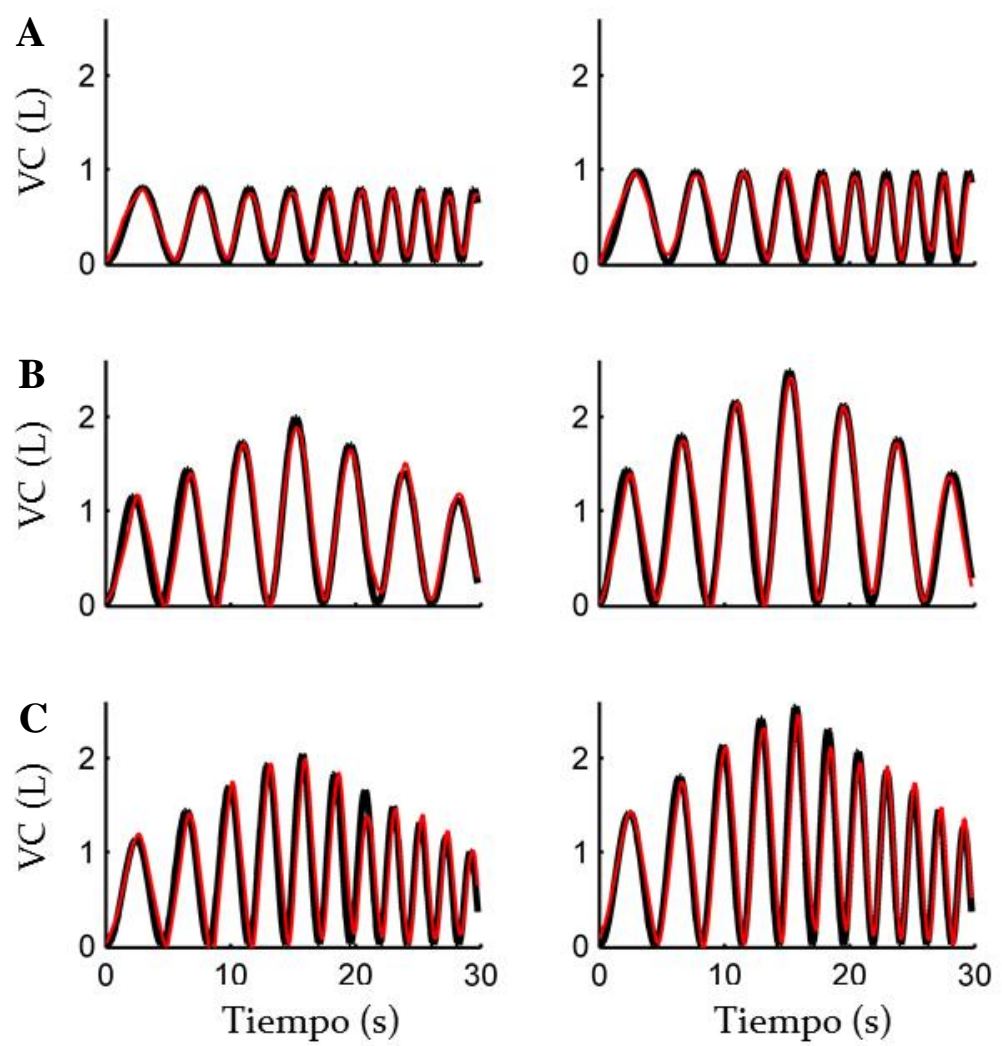

Fig. 41. Superposición de los patrones respiratorios (líneas negras) y los promedios ensamblados de las señales respiratorias registradas (líneas rojas) durante la ejecución de las maniobras cortas en posición sentada: A) $\left.\left.F R_{L I}, B\right) V C_{L I}-V C_{L D}, C\right) ~ F R_{L /} / V_{L I}-V C_{L D}$. Las diferencias entre las líneas parecen pequeñas, lo que indica que los sujetos ejecutaron el control respiratorio adecuadamente.

Los valores de los errores medios entre el patrón a seguir y las señales respiratorias de los sujetos se muestran en la Tabla 2.

Tabla 2. Errores medios entre patrones respiratorios y señales respiratorias registradas.

\section{$\mathrm{FR}_{\mathrm{LI}}$}

$0.11 \pm 0.03$

$0.12 \pm 0.02$
$\mathrm{VC}_{\mathrm{LI}}-\mathrm{VC}_{\mathrm{LD}}$

$0.28 \pm 0.06$

$0.25 \pm 0.04$
$\mathrm{FR}_{\mathrm{LI}} / \mathrm{VC}_{\mathrm{LI}}-\mathrm{VC}_{\mathrm{LD}}$

$0.19 \pm 0.02$

$0.19 \pm 0.03$ 
Las distribuciones tiempo-frecuencia de las series

RR durante las tres condiciones de respiración controlada mostraron que la potencia espectral se distribuyó principalmente en la banda de $\mathrm{AF}_{\mathrm{RR}}$, con poca potencia en la banda de baja frecuencia (Fig. 42). Cada maniobra respiratoria produjo un efecto distintivo en la potencia del componente $\mathrm{AF}_{\mathrm{RR}}$ : reducción progresiva durante $\mathrm{FR}_{\mathrm{LI}}$ (Fig. 42A), cambios inapreciables durante $\mathrm{VC}_{\mathrm{LI}}$ seguidos por reducción gradual durante $\mathrm{VC}_{\mathrm{LD}}$ (Fig. 42B) y una gran reducción de la potencia de $\mathrm{AF}_{\mathrm{RR}}$ durante la maniobra combinada, mayor que la producida por las otras dos maniobras (Fig. 42C).

Los valores medios de $R_{m}$ y $\ln A F_{R R}$ durante el control no fueron diferentes entre hombres $y$ mujeres (Tabla 3) ni entre las tres maniobras. La dinámica de la VP fue distintiva para cada maniobra (Fig. 43A): a lo largo de toda la maniobra $\mathrm{FR}_{\mathrm{LI}}$ se incrementó progresivamente; durante la maniobra
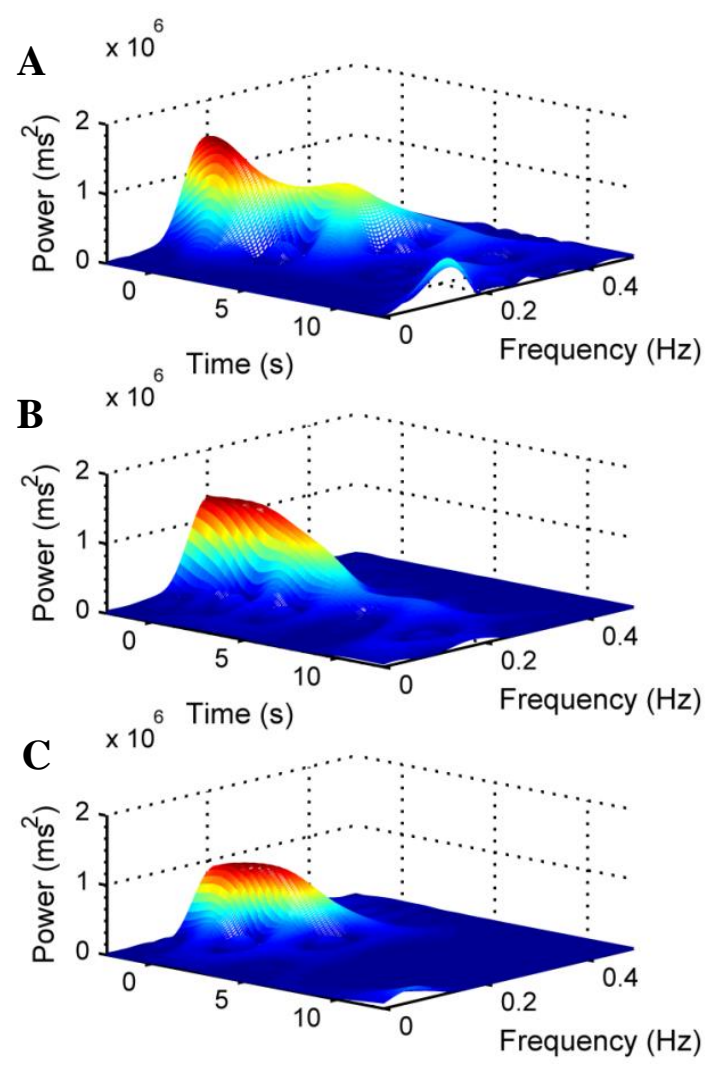

Fig. 42. Ejemplo representativo de las distribuciones tiempo-frecuencia de las series de intervalos $R R$ durante las tres maniobras: a) $\mathrm{FR}_{\mathrm{LI}}$; b) $\left.\mathrm{VC}_{\mathrm{LI}}-\mathrm{VC}_{\mathrm{LD}}, \mathrm{c}\right) \mathrm{FR}_{\mathrm{L}} / \mathrm{VC}_{\mathrm{LI}}-\mathrm{VC}_{\mathrm{LD}}$. Cada maniobra produjo un efecto característico sobre la potencia del componente de $\mathrm{AF}_{\mathrm{Dq}}$.

$\mathrm{VC}_{\mathrm{LI}}-\mathrm{VC}_{\mathrm{LD}}$ cambió en paralelo con el $\mathrm{VC}$ y en la maniobra combinada alcanzó un máximo y permaneció elevada. Con respecto a sus niveles de control, los valores instantáneos de $\mathrm{ETCO}_{2}$, $\mathrm{RR}_{\mathrm{m}} \mathrm{y} \ln \mathrm{AF}_{\mathrm{RR}}$ disminuyeron progresivamente en las tres maniobras, correspondiendo la mayor caída a la maniobra combinada (Fig. 43 B-D). Los valores medios de $\mathrm{ETCO}_{2}, \mathrm{RR}_{\mathrm{m}}$ y $\ln \mathrm{AF}_{\mathrm{RR}}$ durante las maniobras fueron significativamente diferentes de su correspondiente nivel de control $(\mathrm{p}<0.001)$ y entre las maniobras independientes $(\mathrm{p}<0.01)$, exceptuando el $\ln A F_{\mathrm{RR}}$ de los hombres, y fueron menores $(\mathrm{p}<0.005)$ en la maniobra combinada que en las maniobras independientes (Tabla 3). El valor medio de las diferencias control-maniobra de VP y $\mathrm{ETCO}_{2}$ fueron significativamente diferentes entre las tres maniobras $(\mathrm{p}<0.02)$, correspondiendo el mayor valor a la maniobra combinada. Los valores medios de las diferencias control-maniobra de $R_{m}$ y $\ln A F_{R R}$ fueron similares entre las maniobras independientes pero menores $(\mathrm{p}<0.01)$ que aquéllos 
producidos por la maniobra combinada (Tabla 3). En ninguna de las tres maniobras respiratorias los cambios control-maniobra de $\mathrm{ETCO}_{2}, \mathrm{RR}_{\mathrm{m}} \mathrm{y} \ln \mathrm{AF}_{\mathrm{RR}}$ fueron diferentes entre sexos.

Tabla 3. Valores medios \pm D.E. de las medias de las dinámicas de $\mathrm{VP}, \mathrm{ETCO}_{2}, \mathrm{RR}_{\mathrm{m}} \mathrm{y} \ln \mathrm{AF}_{\mathrm{RR}}$ durante el periodo de control y durante la ejecución de las tres maniobras respiratorias, así como de la diferencia entre estos valores en hombres $(\mathrm{N}=20)$ y mujeres $(\mathrm{N}=18)$.

\begin{tabular}{|c|c|c|c|c|c|c|c|}
\hline & \multirow[t]{2}{*}{ Maniobra } & \multicolumn{2}{|c|}{ Control } & \multicolumn{2}{|c|}{ Maniobra } & \multicolumn{2}{|c|}{$\begin{array}{c}\text { Diferencia Control- } \\
\text { Maniobra }\end{array}$} \\
\hline & & Mujeres & Hombres & Mujeres & Hombres & Mujeres & Hombres \\
\hline \multirow{4}{*}{$\begin{array}{c}\mathrm{VP} \\
(\mathrm{L} / \mathrm{min})\end{array}$} & $\mathrm{FR}_{\mathrm{LI}}$ & $9.9 \pm 0.6$ & $12.1 \pm 0.8 \dagger$ & $15.2 \pm 0.5 *+$ & $18.6 \pm 1.1 * \dagger+$ & $-5.3 \pm 0.7 \ddagger$ & $-6.5 \pm 0.8+t$ \\
\hline & $\begin{array}{l}\mathrm{VC}_{\mathrm{LI}^{-}} \\
\mathrm{VC}_{\mathrm{LD}}\end{array}$ & $10.9 \pm 1.1$ & $12.8 \pm 0.8 \dagger$ & $21.7 \pm 0.9 *+$ & $26.7 \pm 0.8 * \dagger+$ & $-10.7 \pm 1.0 \ddagger$ & $-14.0 \pm 1.1 \dagger t$ \\
\hline & $\mathrm{FR}_{\mathrm{LI}^{-}}$ & & & & & & \\
\hline & $\begin{array}{l}\mathrm{VC}_{\mathrm{LI}^{-}} \\
\mathrm{VC}_{\mathrm{LD}}\end{array}$ & $10.8 \pm 1.3$ & $12.8 \pm 0.6 \dagger$ & $33.2 \pm 1.1 *$ & $41.1 \pm 1.2 \dagger *$ & $-22.4 \pm 1.3$ & $-28.3 \pm 1.4 \dagger$ \\
\hline \multirow{4}{*}{$\begin{array}{c}\mathrm{ETCO}_{2} \\
(\%)\end{array}$} & $\mathrm{FR}_{\mathrm{LI}}$ & $5.6 \pm 0.5$ & $6.1 \pm 0.5 \dagger$ & $4.9 \pm 0.5 *+$ & $5.3 \pm 0.5^{*+\dagger}$ & $0.6 \pm 0.2 \ddagger$ & $0.7 \pm 0.2 \ddagger$ \\
\hline & $\begin{array}{l}\mathrm{VC}_{\mathrm{LI}^{-}} \\
\mathrm{VC}_{\mathrm{LD}}\end{array}$ & $5.6 \pm 0.5$ & $5.9 \pm 0.5 \dagger$ & $4.7 \pm 0.5^{*}+$ & $5.0 \pm 0.5^{*+\dagger}$ & $0.9 \pm 0.3 \ddagger$ & $0.9 \pm 0.2 \ddagger$ \\
\hline & $\mathrm{FR}_{\mathrm{LI}^{-}}$ & & & & & & \\
\hline & $\begin{array}{l}\mathrm{VC}_{\mathrm{LI}^{-}} \\
\mathrm{VC}_{\mathrm{LD}}\end{array}$ & $5.2 \pm 0.4$ & $5.6 \pm 0.8 \dagger$ & $3.3 \pm 0.4 *$ & $3.7 \pm 0.6 \dagger *$ & $1.8 \pm 0.2$ & $1.9 \pm 0.3$ \\
\hline \multirow{3}{*}{$\begin{array}{l}\mathrm{RR}_{\mathrm{m}} \\
(\mathrm{ms})\end{array}$} & $\mathrm{FR}_{\mathrm{LI}}$ & $766 \pm 131$ & $811 \pm 113$ & $722 \pm 123 *+$ & $764 \pm 102 * \div$ & $43 \pm 26 \ddagger$ & $47 \pm 33 \div$ \\
\hline & $\begin{array}{l}\mathrm{VC}_{\mathrm{LI}^{-}} \\
\mathrm{VC}_{\mathrm{LD}}\end{array}$ & $740 \pm 109$ & $771 \pm 89$ & $671 \pm 96 *+$ & $698 \pm 85 *+$ & $69 \pm 44 \div$ & $73 \pm 32 \div$ \\
\hline & $\begin{array}{l}\mathrm{FR}_{\mathrm{LI}^{-}} \\
\mathrm{VC}_{\mathrm{LI}^{-}} \\
\mathrm{VC}_{\mathrm{LD}}\end{array}$ & $774 \pm 96$ & $793 \pm 116$ & $664 \pm 78 *$ & $670 \pm 89 *$ & $109 \pm 50$ & $123 \pm 45$ \\
\hline \multirow{3}{*}{$\begin{array}{c}\ln \mathrm{AF}_{\mathrm{RR}} \\
\left(\mathrm{ms}^{2}\right)\end{array}$} & $\mathrm{FR}_{\mathrm{LI}}$ & $7.9 \pm 1.4$ & $8.1 \pm 1.1$ & $7.1 \pm 1.5 *+$ & $7.4 \pm 1.0 *+$ & $0.7 \pm 0.5 t$ & $0.7 \pm 0.5 \ddagger$ \\
\hline & $\begin{array}{l}\mathrm{VC}_{\mathrm{LI}^{-}} \\
\mathrm{VC}_{\mathrm{LD}}\end{array}$ & $8.1 \pm 1.2$ & $8.2 \pm 0.8$ & $7.5 \pm 1.3 * t$ & $7.9 \pm 1.0 * t$ & $0.6 \pm 0.6+$ & $0.4 \pm 0.5 t$ \\
\hline & $\begin{array}{l}\mathrm{FR}_{\mathrm{LI}^{-}} \\
\mathrm{VC}_{\mathrm{LI}^{-}} \\
\mathrm{VC}_{\mathrm{LD}}\end{array}$ & $8.2 \pm 1.0$ & $8.1 \pm 1.1$ & $6.8 \pm 1.2 *$ & $6.9 \pm 1.2 *$ & $1.3 \pm 0.8$ & $1.2 \pm 0.6$ \\
\hline
\end{tabular}

$* \mathrm{p}<0.01$ Maniobra vs. control

$\dagger \mathrm{p}<0.01$ Hombres vs. Mujeres

$\$ \mathrm{p}<0.01$ Maniobras Independientes vs. Combinada 

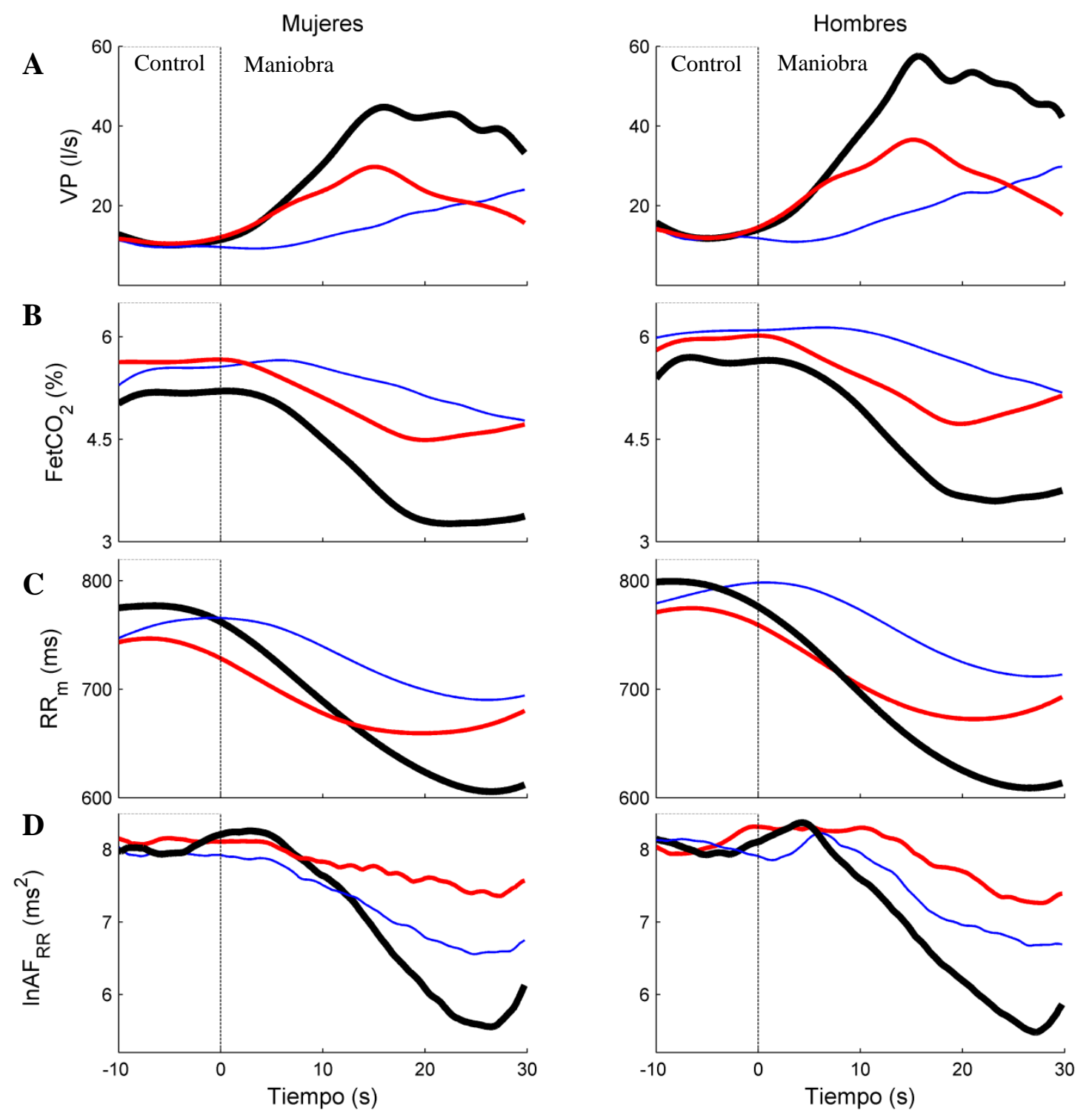

Fig. 43. Promedios ensamblados de las dinámicas de las variables respiratorias y cardiovasculares durante las tres maniobras respiratorias: $\mathrm{FR}_{\mathrm{LI}}$ (línea azul), $\mathrm{VC}_{\mathrm{LI}} \mathrm{VC}_{\mathrm{LD}}$ (línea roja) y $\mathrm{FR}_{\mathrm{LI}} / \mathrm{VC}_{\mathrm{LI}} \mathrm{VC}_{\mathrm{LD}}$ (línea negra).

Es notable que los valores medios de $\mathrm{RR}_{\mathrm{m}}$ y $\ln \mathrm{AF}_{\mathrm{RR}}$ producidos por la maniobra combinada resultaran muy cercanos a la suma de los valores medios correspondientes a cada maniobra independiente tanto en hombres como mujeres, a pesar de que la VP fuera mayor en los primeros. Los valores de los II calculados para los dos indicadores de ASR utilizados fueron muy cercanos a uno para ambos sexos, indicando que la interacción del VC y el FR sobre los núcleos autonómicos generadores de la ASR es aditiva (Fig. 44). 

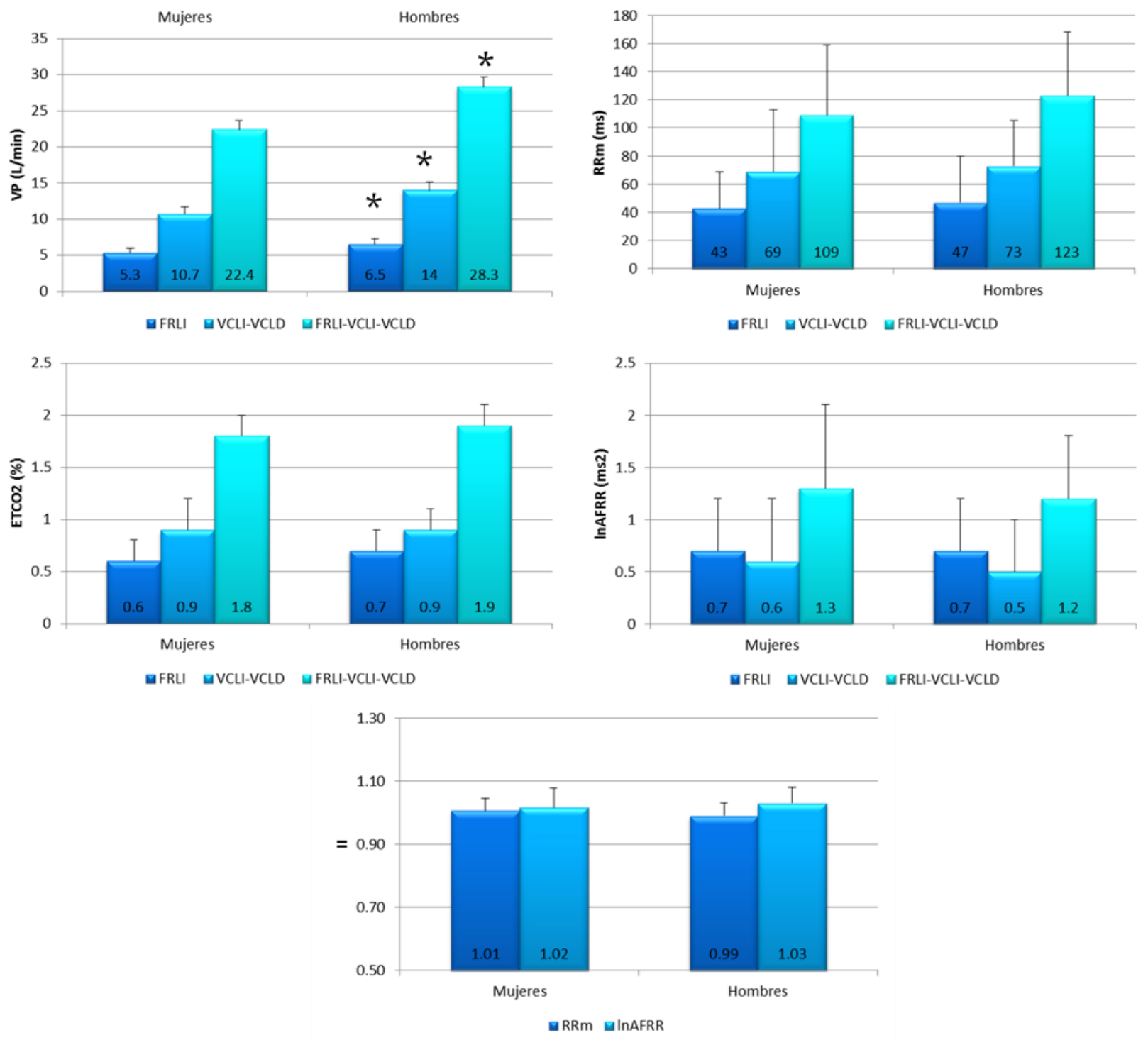

Fig. 44. Comparación de los valores medios de las variables respiratorias y cardiovasculares. Es notable que los valores medios del $R_{m}$ y el $I n A F_{R R}$ durante la maniobra combinada equivalgan a la suma algebraica de los valores medios durante las maniobras independientes. Con excepción de VP, no hubo diferencias significativas entre hombres y mujeres. ${ }^{*} p<0.05$ Hombres vs. Mujeres.

La Fig. 45 muestra los promedios ensamblados de las relaciones $\ln \mathrm{AF}_{\mathrm{RR}}-\mathrm{FR}_{\mathrm{LI}}, \ln \mathrm{AF}_{\mathrm{RR}}-\mathrm{VC}_{\mathrm{LI}} \mathrm{y}$ $\ln \mathrm{AF}_{\mathrm{RR}}-\mathrm{VC}_{\mathrm{LD}}$ con sus respectivas regresiones lineales durante las maniobras independientes y combinadas.

$\mathrm{FR}_{\mathrm{LI}}$ indujo una reducción lineal de $\ln \mathrm{AF}_{\mathrm{RR}}$ sistemática tanto en la maniobra independiente como en la combinada (Fig. 45A), con mayores pendientes ( $\mathrm{p}<0.001$ ) en esta última (Tabla 4). 

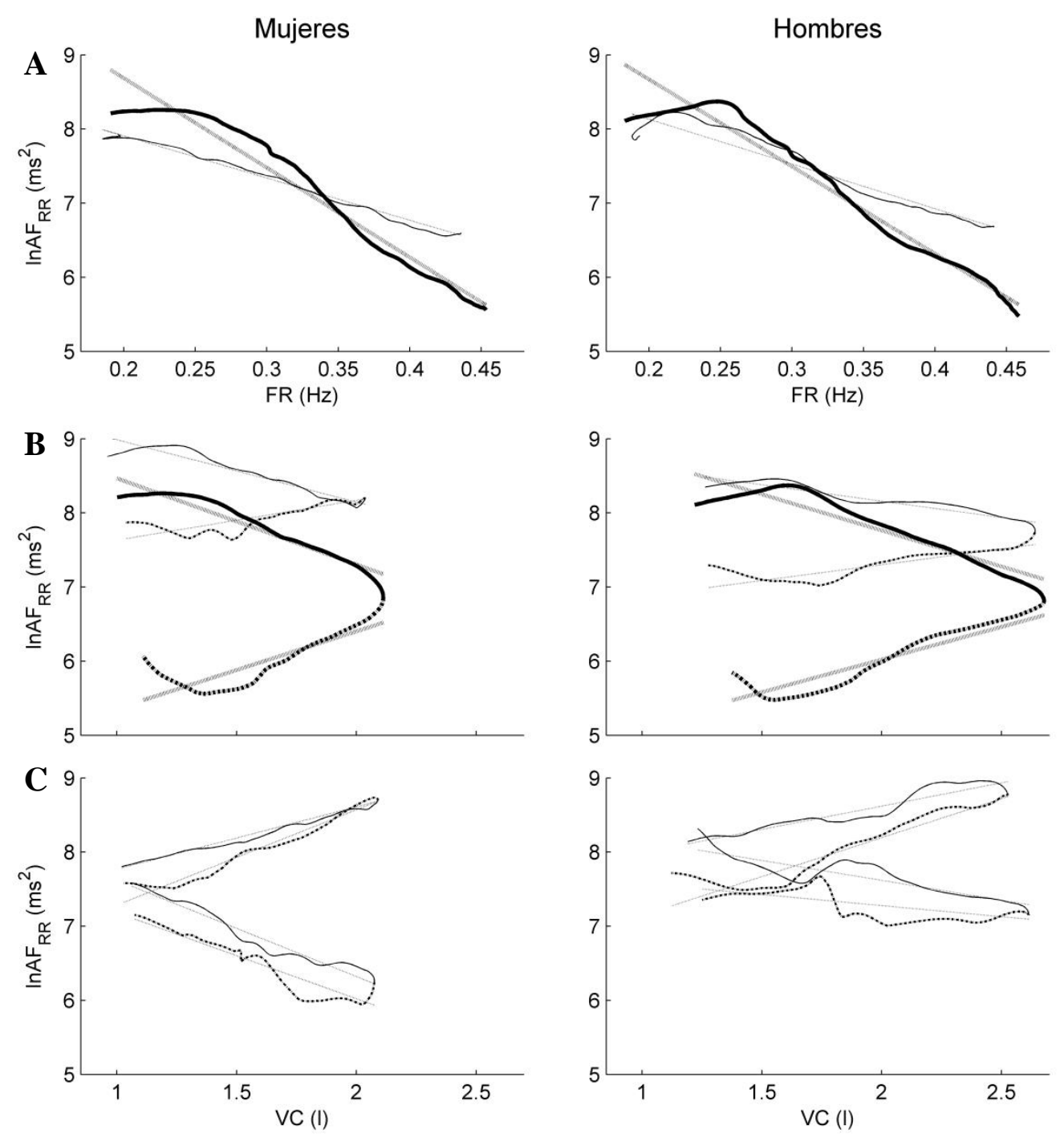

Fig. 45. Relaciones entre el indicador espectral de ASR y las variables respiratorias, construidas a partir de sus promedios ensamblados y superpuestas con las correspondientes regresiones medias. A) InAF $F_{R R}-F R$. La línea continua delgada corresponde a la maniobra $F R_{L I}$ y la línea continua gruesa a la maniobra $F R_{L I} / V_{L^{-}}$ $V C_{L D}$. B) Caso más frecuente en la relación $I n A F_{R R}-V C$, durante la maniobra $V C_{L I}-V C_{L D}$, junto con la respuesta a la maniobra $\mathrm{FR}_{\mathrm{L}} / \mathrm{VC}_{\mathrm{LI}}-\mathrm{VC}_{\mathrm{LD}}$, que consistieron en una relación lineal negativa durante la parte incremental y una relación lineal con pendiente positiva en la parte decremental. C) Los dos casos restantes de relaciones $I n A F_{R R}-V C$ en respuesta a la maniobra $V C_{L I}-V_{L D}$ : pendientes positivas tanto en la fase creciente como en la decreciente y pendientes negativas en las dos fases.

El efecto de $\mathrm{VC}_{\mathrm{LI}}-\mathrm{VC}_{\mathrm{LD}}$ sobre $\ln \mathrm{AF}_{\mathrm{RR}}$ no fue consistente durante la maniobra independiente, la cual produjo tres respuestas diferentes: 1) en $49 \%$ de los sujetos estudiados, la relación entre $\operatorname{lnAF} F_{R R}$ y $V C$ presentó una pendiente negativa en la parte $\mathrm{VC}_{\mathrm{LI}}$ y una pendiente positiva en la sección $\mathrm{VC}_{\mathrm{LD}}$, con pendientes e interceptos significativamente diferentes $(\mathrm{p}<0.001$, Tabla 4, Fig. 45B); 2) en $28 \%$ de los voluntarios ambas relaciones fueron positivas pero con diferencias significativas ( $\mathrm{p}<0.01$ ) en pendientes e interceptos (Tabla 4, Fig. 45C); 3) en $23 \%$ de los sujetos 
ambas relaciones fueron negativas (Tabla 4, Fig. 45C), con pendientes similares pero interceptos diferentes $(\mathrm{p}<0.01)$.

Tabla 4. Medias \pm D.E. de las correlaciones, pendientes e interceptos de las relaciones entre $\ln \mathrm{AF}_{\mathrm{RR}}$ y FR durante las maniobras independiente (I) y combinada (C) para mujeres $(\mathrm{N}=18)$ y hombres $(\mathrm{N}=20)$.

\begin{tabular}{ccccccc}
\hline \multirow{2}{*}{ Maniobra } & \multicolumn{2}{c}{ Correlación } & \multicolumn{2}{c}{ Pendiente } & \multicolumn{2}{c}{ Intercepto } \\
& Mujeres & Hombres & Mujeres & Hombres & Mujeres & Hombres \\
\hline I & $-0.91 \pm 0.10$ & $-0.91 \pm 0.10$ & $-6.5 \pm 3.4^{*}$ & $-6.3 \pm 3.9^{*}$ & $10.9 \pm 1.7$ & $10.9 \pm 1.5$ \\
C & $-0.91 \pm 0.08$ & $-0.91 \pm 0.07$ & $-12.1 \pm 5.1$ & $-11.8 \pm 3.6$ & $11.1 \pm 1.9$ & $11.0 \pm 1.5$ \\
\hline
\end{tabular}

*p $<0.01$ maniobra independiente vs. Combinada.

Todas las correlaciones fueron significativas $(\mathrm{p}<0.01)$.

La maniobra combinada produjo un efecto sistemático en la relación $\ln \mathrm{AF}_{\mathrm{RR}}-\mathrm{VC}_{\mathrm{LI}}-\mathrm{VC} \mathrm{CD}_{\mathrm{LD}}$. Durante la parte $\mathrm{VC}_{\mathrm{LI}}$ tuvo una pendiente negativa, de mayor valor $(\mathrm{p}<0.01)$ que cualquiera de las pendientes correspondientes a los tres casos producidos por la maniobra independiente (Tabla 4). Durante la sección correspondiente a $\mathrm{VC}_{\mathrm{LD}}$, la pendiente fue positiva y más grande $(\mathrm{p}<0.01)$ y el intercepto fue menor $(\mathrm{p}<0.01)$ que los de las maniobras independientes (Tabla 4). Los tres tipos de relaciones entre $\ln \mathrm{AF}_{\mathrm{RR}}$ y $\mathrm{VC}_{\mathrm{LI}}-\mathrm{VC}_{\mathrm{LD}}$ presentaron histéresis, con valores medios de $\Delta \mathrm{I}$ de $2.0 \pm 1.5$ $\mathrm{ms}^{2}$ para mujeres y $2.2 \pm 1.2 \mathrm{~ms}^{2}$ para hombres. Las respuestas de la potencia de $\ln A F_{\mathrm{RR}}$ a la variación simultánea de FR y VC presentaron una histéresis considerablemente mayor que las independientes, reflejada por los valores medios de $\Delta \mathrm{I}, 5.3 \pm 1.8 \mathrm{~ms}^{2}$ para mujeres y $5.5 \pm 1.7$ para hombres, ambos mayores que los correspondientes a las condiciones independientes $(\mathrm{p}<0.001)$. Los coeficientes de correlación, las pendientes y los interceptos de las relaciones de $\ln \mathrm{AF}_{\mathrm{RR}}$ con $\mathrm{FR}_{\mathrm{LI}}$ y $\mathrm{VC}_{\mathrm{LI}}-\mathrm{VC}_{\mathrm{LD}}$ obtenidos durante las maniobras independientes y combinadas no presentaron diferencias significativas entre sexos. Las correlaciones de las relaciones $\ln A F_{R R^{-}}-F_{L I}, \ln A F_{R^{-}}$ $\mathrm{VC}_{\mathrm{LI}}$ y $\ln \mathrm{AF}_{\mathrm{RR}}-\mathrm{VC}_{\mathrm{LD}}$ variaron entre 0.79 y 0.95 , con excepción de aquéllos con respuestas a $\mathrm{VC}_{\mathrm{LD}}$ tipo 3 , que tuvieron menores correlaciones (Tablas 4 y 5 ). 
Tabla 5. Medias \pm D.E. de la correlaciones, pendientes e interceptos de las relaciones entre la potencia de $\ln \mathrm{AF}_{\mathrm{RR}}$ y el $\mathrm{VC}$, computados para las dos modalidades de variación de este último, incremental (INC) y decremental (DEC) y agrupados en los tres casos distintivos que se presentaron durante las maniobras respiratorias independiente (I) y combinada (C) para mujeres $(\mathrm{N}=18)$ y hombres $(\mathrm{N}=20)$.

\begin{tabular}{|c|c|c|c|c|c|c|c|c|}
\hline \multirow{2}{*}{ Maniobra } & \multirow{2}{*}{ Caso } & \multirow{2}{*}{$\begin{array}{c}\text { Modalidad } \\
\text { VC }\end{array}$} & \multicolumn{2}{|c|}{ Correlación } & \multicolumn{2}{|c|}{ Pendiente } & \multicolumn{2}{|c|}{ Intercepto } \\
\hline & & & Mujeres & Hombres & Mujeres & Hombres & Mujeres & Hombres \\
\hline \multirow{6}{*}{ I } & \multirow{2}{*}{1} & INC & $-0.83 \pm 0.14$ & $-0.85 \pm 0.13$ & $-0.7 \pm 0.5 \dagger^{*}$ & $-0.5 \pm 0.4 \dagger^{*}$ & $10.5 \pm 1.4 \dagger$ & $10.4 \pm 1.1 \dagger$ \\
\hline & & DEC & $0.93 \pm 0.04 *$ & $0.79 \pm 0.22$ & $0.9 \pm 0.2 *$ & $0.5 \pm 0.4^{*}$ & $7.4 \pm 1.3 *$ & $7.6 \pm 1.8 *$ \\
\hline & \multirow{2}{*}{2} & INC & $0.83 \pm 0.18^{*}$ & $0.87 \pm 0.12 *$ & $0.9 \pm 0.6+*$ & $0.6 \pm 0.4 \dagger *$ & $6.9 \pm 1.1 \dagger^{*}$ & $9.1 \pm 1.1 \dagger^{*}$ \\
\hline & & $\mathrm{DEC}$ & $0.88 \pm 0.16^{*}$ & $0.90 \pm 0.17 *$ & $1.1 \pm 0.5$ & $0.9 \pm 0.6$ & $8.4 \pm 1.1 *$ & $7.2 \pm 1.4 *$ \\
\hline & \multirow{2}{*}{3} & INC & $-0.95 \pm 0.03^{*}$ & $-0.93 \pm 0.03 *$ & $-0.9 \pm 0.9+*$ & $-0.5 \pm 0.2 \dagger^{*} *$ & $10.6 \pm 2.1$ & $10.1 \pm 0.7$ \\
\hline & & DEC & $-0.70 \pm 0.21$ & $-0.55 \pm 0.17 *$ & $-0.7 \pm 0.2^{*}$ & $-0.3 \pm 0.2 *$ & $9.4 \pm 1.1 *$ & $9.1 \pm 0.7 *$ \\
\hline \multirow{2}{*}{$\mathrm{C}$} & & INC & $-0.82 \pm 0.13$ & $-0.88 \pm 0.10$ & $-1.4 \pm 0.9 \dagger$ & $-1.0 \pm 0.4 \dagger$ & $9.9 \pm 1.5 \dagger$ & $9.7 \pm 1.2 \dagger$ \\
\hline & & $\mathrm{DEC}$ & $0.78 \pm 0.16$ & $0.81 \pm 0.20$ & $1.4 \pm 0.6$ & $1.1 \pm 0.6$ & $4.3 \pm 1.6$ & $4.3 \pm 1.6$ \\
\hline
\end{tabular}

$* \mathrm{p}<0.001$ Maniobra independiente vs. Combinada

$\dagger \mathrm{p}<0.01 \mathrm{VC}_{\mathrm{LI}}$ vs. $\mathrm{VC}_{\mathrm{LD}}$

Todas las correlaciones fueron significativas $(\mathrm{p}<0.01)$. 


\section{Discusión}

\section{Principales hallazgos}

Los principales hallazgos de la presente investigación son:

1) Los efectos de las maniobras respiratorias independientes y combinadas sobre el $\ln A F_{R R}$ no muestran diferencias significativas entre hombres y mujeres.

2) La relación continua, lineal e inversa entre $\ln A F_{R R}$ y $F_{L I}$ presenta una pendiente mayor durante la maniobra combinada que durante la independiente.

3) Los efectos de la maniobra $\mathrm{VC}_{\mathrm{LI}}-\mathrm{VC}_{\mathrm{LD}}$ independiente no son consistentes, produciendo diferentes relaciones lineales entre $\ln \mathrm{AF}_{\mathrm{RR}}$ y $\mathrm{VC}$ que muestran histéresis. El efecto más común de $\mathrm{VC}_{\mathrm{LI}}$ es una relación lineal inversa y de $\mathrm{VC}_{\mathrm{LD}}$ es una relación lineal directa.

4) La combinación de $\mathrm{FR}_{\mathrm{LI}}$ con $\mathrm{VC}_{\mathrm{LI}}-\mathrm{VC}_{\mathrm{LD}}$ induce una reducción de la potencia de $\mathrm{AF}_{\mathrm{RR}}$ equivalente a la suma de las inhibiciones producidas por cada maniobra independiente. Este mismo efecto es observado en $\mathrm{RR}_{\mathrm{m}}$, y sugiere una interacción aditiva entre ambos estímulos.

5) Los efectos de la variación simultánea de la FR y el VC sobre el $\ln \mathrm{AF}_{\mathrm{RR}}$ son sistemáticos, formando un patrón que consiste en una sección linealmente decreciente, correspondiente a $\mathrm{VC}_{\mathrm{LI}}$, seguida por una sección lineal positiva asociada a $\mathrm{VC}_{\mathrm{LD}}$. La histéresis de la relación $\ln \mathrm{AF}_{\mathrm{RR}}-\mathrm{VC}$ en la maniobra combinada es $244 \pm 92 \%$ mayor que la producida por las maniobras independientes, lo que indica una atenuación de la potencia de $\mathrm{AF}_{\mathrm{RR}}$ mucho mayor. Todos estos hallazgos sugieren que los efectos del VC y la FR sobre los núcleos vagales se ejercen por vías independientes.

\section{Sobre las maniobras}

La duración de las maniobras respiratorias diseñadas para este proyecto se ajustó a 150 y 30 segundos para minimizar la fatiga respiratoria y la hiperventilación de los sujetos, y, en el último caso, para permitir la ejecución simultánea de respiración controlada y otras maniobras fisiológicas. La FR fue continuamente incrementada y el VC fue cambiado respiración a respiración de tal manera que las relaciones entre estas variables y la potencia de $\mathrm{AF}_{\mathrm{RR}}$ fuera lo más continuas posible. Además, el VC fue incrementado y reducido para evaluar la ocurrencia de histéresis en la relación $\ln \mathrm{AF}_{\mathrm{RR}}-\mathrm{VC}$. Adicionalmente, los efectos simultáneos de la FR y el VC en 
la amplitud de la ASR fueron explorados mediante la combinación de los cambios continuos y lineales de las dos variables respiratorias. Las diferencias notablemente pequeñas entre el patrón a seguir y los registros del $\mathrm{VC}$ de los sujetos soportan que las maniobras respiratorias fueron ejecutadas correctamente (Figs. 41 y 46 ).

Los efectos independientes de la FR sobre la amplitud de la ASR han sido estudiados con maniobras respiratorias que controlan la FR en un rango seleccionado arbitrariamente y en dos modalidades (Fig. 46): fluctuaciones pseudo-aleatorias (Saul et al. 1989) e incrementos escalonados (Cooke et al. 1998). En el primer tipo de maniobra respiratoria, que es muy difícil de realizar correctamente, el VC no es controlado. El segundo tipo de maniobra es muy fatigante, ya que se deben mantener distintas FR discontinuas durante varios minutos cada una (Taylor et al. 2001). En contraste, la condición $\mathrm{FR}_{\mathrm{LI}}$ empleada en el presente estudio es fácilmente ejecutable, según confirman los pequeños errores obtenidos en las FR y VC instantáneos e incluye un amplio rango de FR variadas continua y linealmente en un tiempo muy corto, minimizando así la hiperventilación y fatiga del sujeto (Fig. 46).

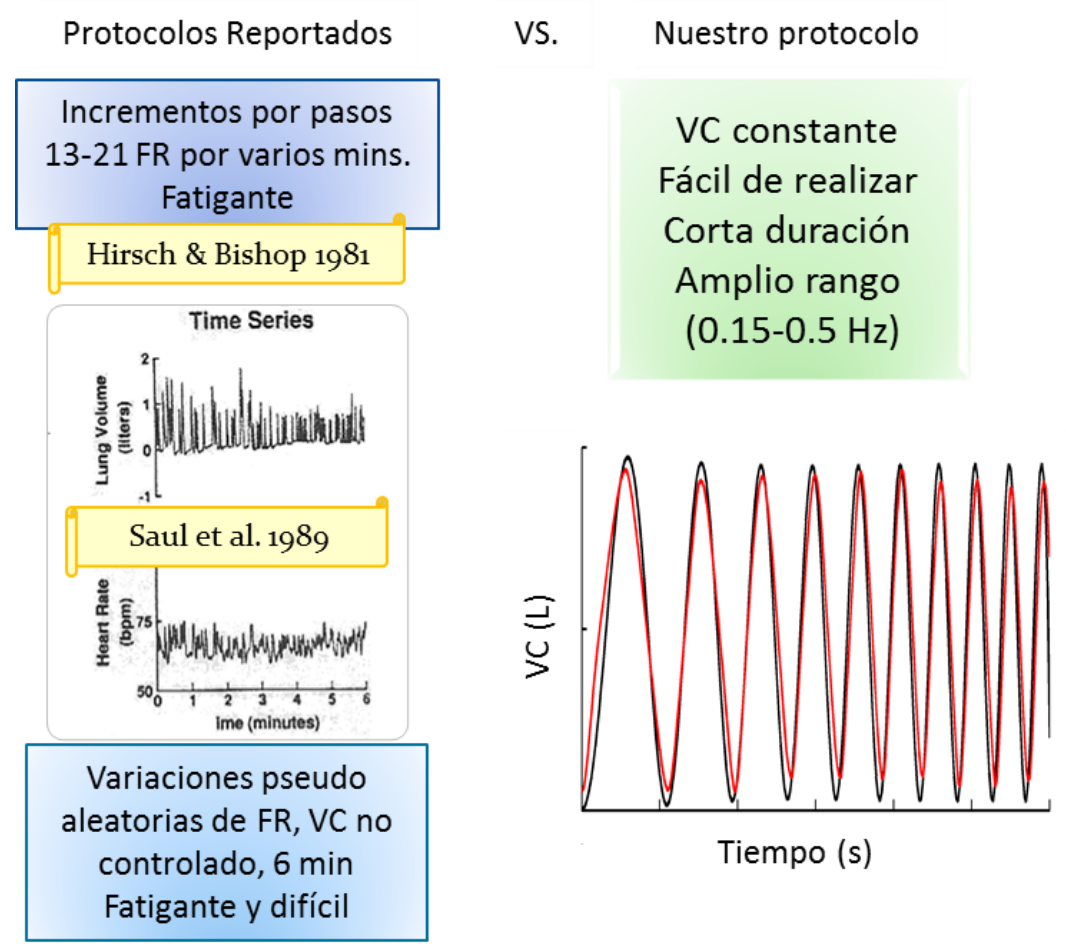

Fig. 46. Los protocolos de respiración controlada utilizados en los estudios reportados acerca del efecto de la FR sobre la amplitud de la ASR son usualmente muy largos y difíciles de ejecutar, ya sea variándola por pasos o de manera aleatoria, además de que comprenden pocas FR y generalmente no controlan el VC. En contraste, el protocolo $\mathrm{FR}_{\mathrm{LI}}$ que se presenta aquí abarca un rango amplio de $\mathrm{FR}$ en un tiempo corto, por lo que, además de resultar cómodo y fácil de seguir para el voluntario, provee más información en un formato continuo y variante en el tiempo. 
Todos los estudios que buscan establecer el efecto del VC sobre la ASR han empleado condiciones similares, las cuales consisten en por lo menos seis maniobras con VC crecientes a una FR fija, cada una sostenida durante varios minutos (Kobayashi 1998, Larsen et al. 2010). En contraste, en nuestra condición a FR fija el VC es linealmente incrementado hasta un máximo y después linealmente reducido respiración por respiración. Dada su corta duración, esta maniobra no es fatigante, es relativamente fácil de realizar y es capaz de evaluar la ocurrencia de histéresis en la respuesta (Fig. 47).

Protocolos reportados

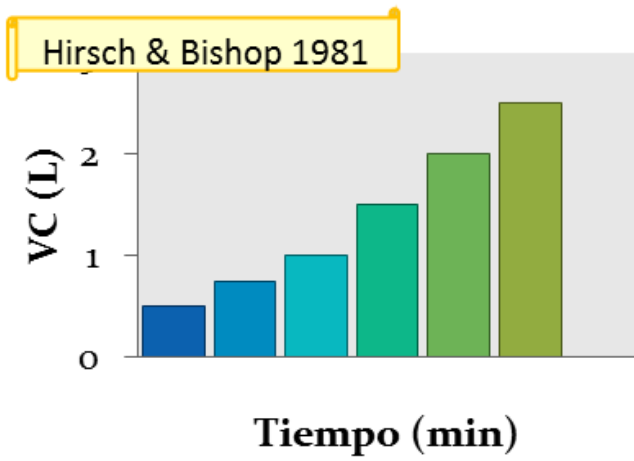

Diferentes VC a FR fija por algunos minutos. Repetir para otros FR y VC

Kobayashi 1998
VS.

Nuestro protocolo

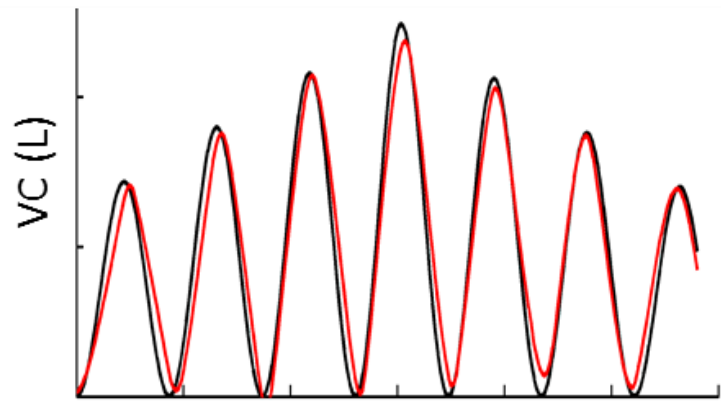

Tiempo(s)

FR fija, VC linealmente incrementadodecrementado $\rightarrow$ evaluación de histéresis Cambio respiración a respiración Corta duración $\rightarrow$ menos hiperventilación y fatiga

Fig. 47. Los escasos estudios que han abordado la relación entre la amplitud de la ASR y el VC han utilizado el mismo estilo de protocolo: sostener un VC a una cierta FR durante algunos minutos, repitiéndolo para diferentes valores de VC arbitrariamente seleccionados. Este tipo de maniobras resulta agotador para los sujetos de estudio, y como resultado, se obtienen pocos valores puntuales. En cambio, el protocolo de variación respiración a respiración del VC en forma creciente y decreciente en un tiempo corto resulta más sencillo de ejecutar y permite obtener más información, ya que permite construir relaciones continuas variantes en el tiempo con un amplio rango de valores, así como evaluar la existencia de histéresis.

\section{Efectos de la variación independiente de FR}

La relación inversamente proporcional entre $\ln \mathrm{AF}_{\mathrm{RR}}$ y $\mathrm{FR}$, conocida como el efecto como-filtropasa-bajas del sistema formado por el SNA y el nodo SA, ha sido ampliamente documentada. La relación continua que produjo la maniobra $\mathrm{FR}_{\mathrm{LI}}$ propuesta es similar a las reportadas (Fig. 48). Por 
lo tanto, los resultados de esta investigación refuerzan que la atenuación de la amplitud de la ASR causada al incrementar continua y linealmente la FR es un efecto marcadamente consistente y reproducible, sin importar el tipo de maniobra respiratoria utilizada.

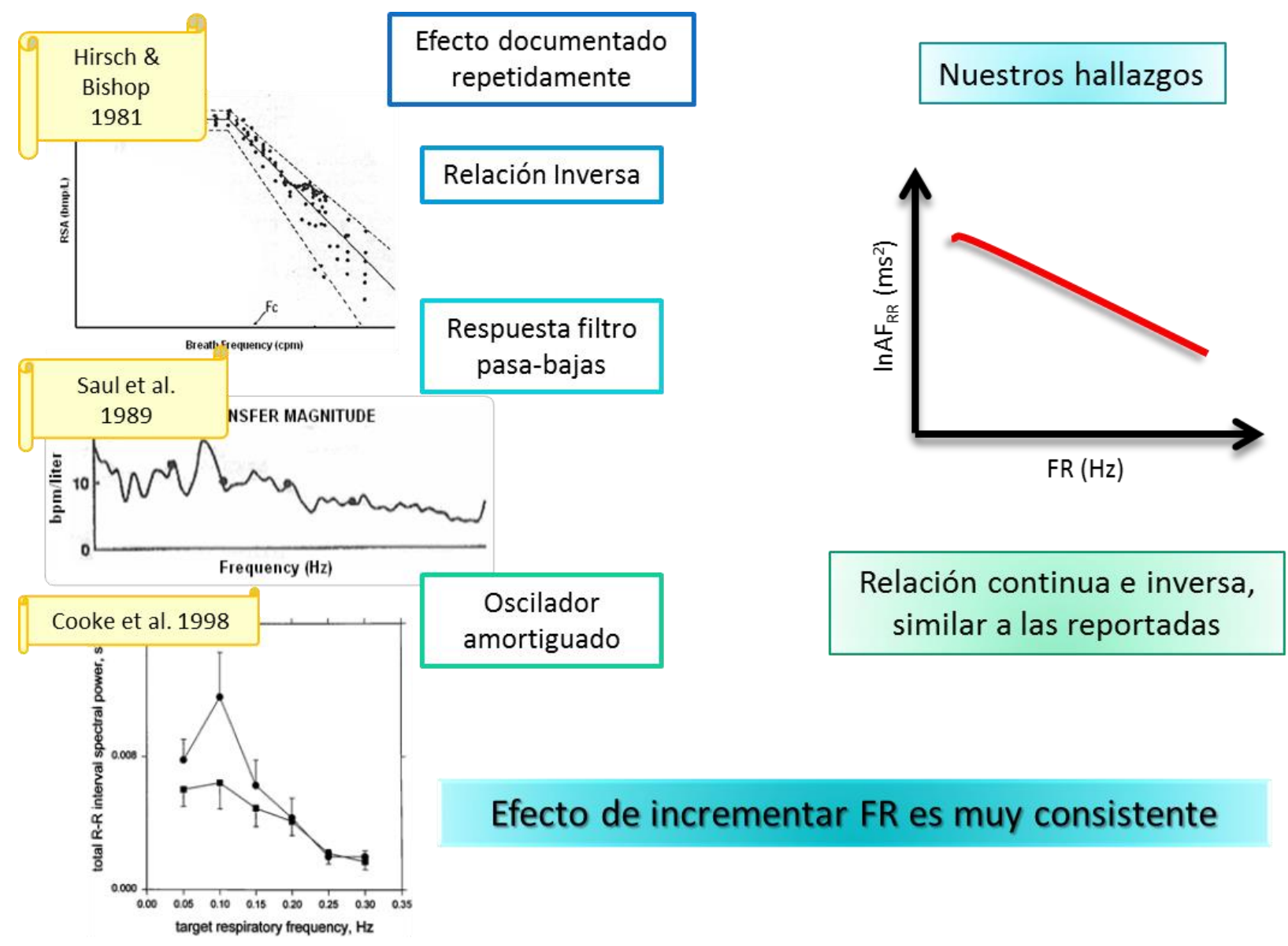

Fig. 48. Múltiples estudios han reportado la relación inversa que existe entre la FR y la amplitud de la ASR, evaluada por el $R_{m}$ o por la potencia de $A F_{R R}$. Esta relación ha sido descrita como similar a la respuesta de un filtro pasa-bajas o como un oscilador amortiguado. Los resultados obtenidos en el presente proyecto fueron similares a los reportados, lo que refuerza la noción de que incrementar la FR ocasiona la atenuación de la amplitud de la ASR, sin importar el tipo de maniobra de respiración controlada utilizado.

La técnica de análisis espectral empleada en la presente investigación permitió evaluar la relación

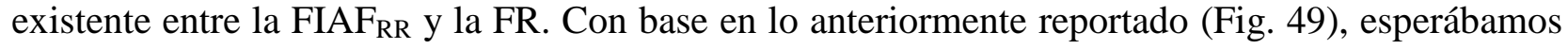
encontrar una relación lineal directa cercana a la línea de identidad. Sorprendentemente, encontramos que la relación $\mathrm{FIAF}_{\mathrm{RR}}-\mathrm{FR}$ se alejó rápidamente de la línea de identidad. La incapacidad de la FIAF $_{R R}$ de seguir a la FR probablemente se deba a un retraso de la cinética de la acetilcolina en los miocitos del nodo sinusal conforme aumenta la FR (Rocchetti et al. 2002), que podría originar la diferencia entre las frecuencias FIAF $_{R R}$ y FR (Fig. 37). Previamente se ha 
reportado (Witte et al. 1988, Grossman y Taylor 2007) que cuando la FR es mayor a la mitad de la frecuencia cardiaca se manifiesta el fenómeno de "aliasing cardiaco": al existir menos de dos latidos por cada ciclo respiratorio, la modulación respiratoria del periodo cardiaco ya no se puede identificar en el tacograma, por lo que la FR y la FIAF $_{R R}$ dejan de ser coherentes entre sí (Fig. 49). Según nuestros resultados, al variar conjuntamente la FR y el VC el aliasing cardiaco se presenta a valores de FR cercanos a los que comúnmente se utilizan en los protocolos de respiración controlada. Este hallazgo, no reportado previamente, reta al concepto de ASR: ¿cuál sería el rango mínimo de diferencia entre la relación FIAF $_{\mathrm{RR}}-\mathrm{FR}$ y la línea de identidad para seguir considerando al fenómeno como ASR? Podría definirse en términos de la coherencia entre los espectros de la VFC y la respiración, de manera similar a los criterios para el cómputo de la sensibilidad del barorreflejo (LeMarbre et al. 2003). Asimismo, resalta la importancia de evaluar la ASR en el mayor rango posible de valores.
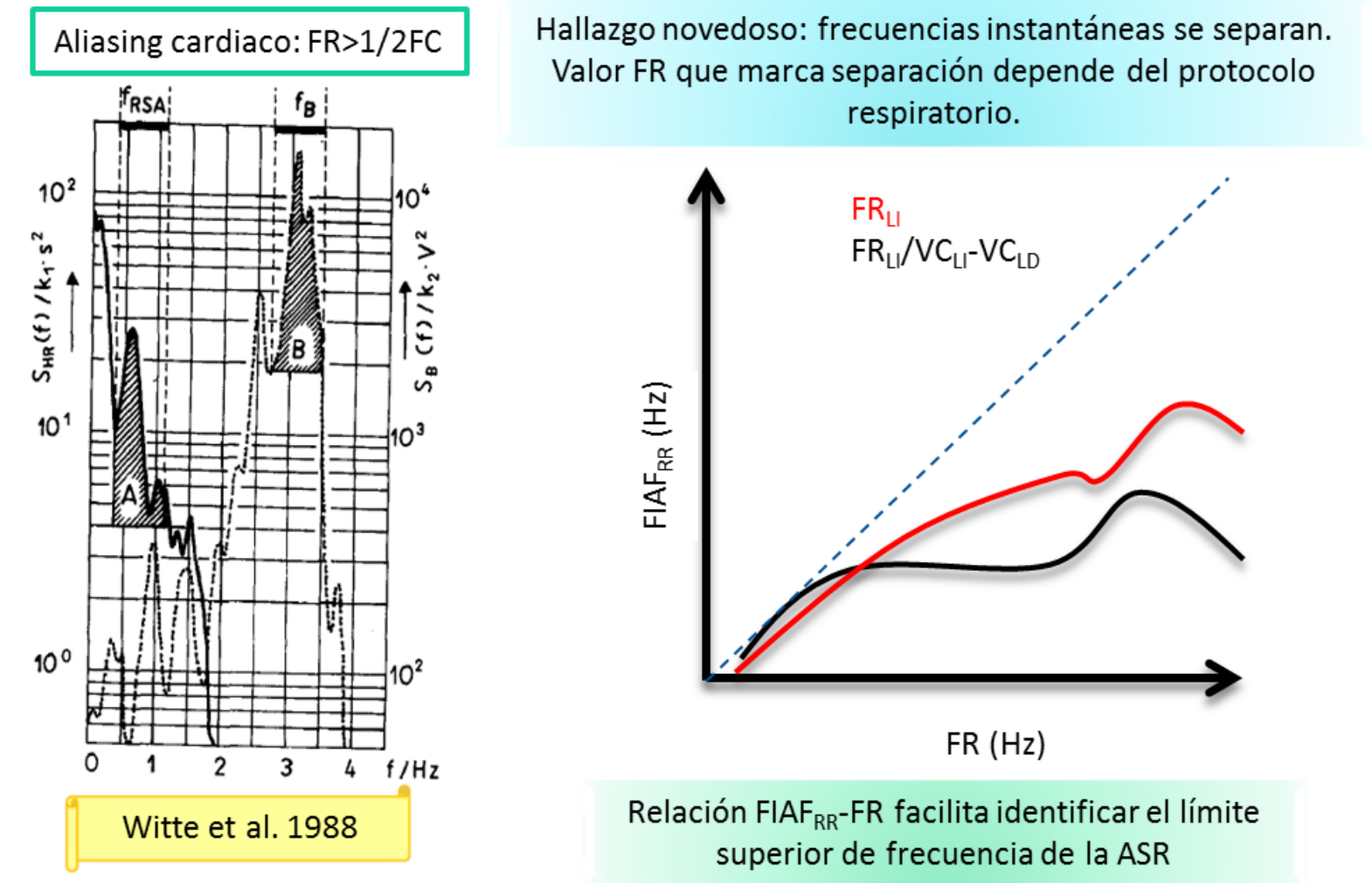

Fig. 49. El fenómeno descrito como "aliasing cardiaco" marca el límite en frecuencia máximo donde tiene sentido calcular la ASR: cuando la FR es superior a la mitad de la frecuencia cardiaca. Gracias al método de análisis espectral empleado, fue posible calcular la relación entre las dinámicas de la FIAFRR y la FR, que permite identificar de manera gráfica y sencilla el punto de inicio del aliasing cardiaco. La variación simultánea de FR y VC tuvo el efecto de recorrer este valor límite hacia la izquierda. 


\section{Efectos de la variación independiente del VC sobre la amplitud de la}

\section{ASR}

Comúnmente se acepta que la magnitud de la ASR se incrementa proporcionalmente a los incrementos en VC (Fig. 50). La linealidad de esta relación es la premisa que avala la normalización de la amplitud de la ASR con respecto al VC, procedimiento ampliamente realizado empleando diversas técnicas (Grossman y Taylor 2007, Kobayashi 1998).

Las diferentes relaciones entre la potencia de $\ln \mathrm{AF}_{\mathrm{RR}}$ y el $\mathrm{VC}$ que obtuvimos durante la aplicación de las diversas maniobras diseñadas (Figs. 35, 36, 40, 45) resultan relevantes, ya que difieren de lo publicado previamente.

Tanto en los estudios piloto como en el estudio definitivo, durante las maniobras de variación del VC a FR constante para la evaluación fisiológica, en los primeros ciclos respiratorios la potencia de $\ln \mathrm{AF}_{\mathrm{RR}}$ se incrementó proporcionalmente al $\mathrm{VC}_{\mathrm{LI}}$ hasta llegar a un $\mathrm{VC}$ umbral ubicado entre 1.2 y $1.6 \mathrm{~L}$, a partir del cual y a pesar de que el VC continuó incrementándose, el valor de $\ln \mathrm{AF}_{\mathrm{RR}}$ permaneció constante o incluso disminuyó. Más aún, durante la fase de $\mathrm{VC}_{\mathrm{LD}}$ la potencia de $\ln \mathrm{AF}_{\mathrm{RR}}$ disminuyó todavía más pero con diferentes valores a los correspondientes al mismo VC en la fase incremental (Fig. 36). Estos resultados indican que: a) existe un umbral máximo de VC, más allá del cual la asunción de que la amplitud de la ASR es directamente proporcional al VC deja de ser válida; b) que la respuesta del $\ln A F_{R R}$ a los cambios lineales respiración a respiración del VC depende no sólo del valor del VC sino también de la dirección del cambio, es decir, presenta histéresis.

Durante las maniobras diseñadas para servir como indicador vagal (Fig. 40), esta respuesta no proporcional y con histéresis se mantuvo, acentuándose aún más estas características durante la postura erguida. La relación $\ln \mathrm{AF}_{\mathrm{RR}}-\mathrm{VC}$ no mostró un comportamiento sistemático durante las maniobras $\mathrm{VC}_{\mathrm{LI}}-\mathrm{VC}_{\mathrm{LD}}$ a $\mathrm{FR}$ constante. Mientras que en $72 \%$ de los participantes de este estudio $\mathrm{VC}_{\mathrm{LI}}$ produjo una reducción lineal de la ASR, opuesta al efecto reportado al incrementar el VC (Fig.45 b-c, casos 1 y 3, Tabla 5), sólo el 28\% respondió con el incremento lineal esperado (Fig. 45c, caso 2, Tabla 5). Durante $\mathrm{VC}_{\mathrm{LD}}$, la amplitud de la ASR disminuyó proporcionalmente en $77 \%$ de los sujetos (Fig. 45 b-c, Tabla 5). Además, en todos los sujetos observamos que, para un nivel dado de $\mathrm{VC}$, la potencia de $\ln \mathrm{AF}_{\mathrm{RR}}$ correspondiente a la sección creciente fue mayor que la correspondiente a la parte decremental, esto es, que la relación $\ln \mathrm{AF}_{\mathrm{RR}}-\mathrm{VC}$ presenta histéresis (Fig. 45 b-c). 
Así, nuestros resultados documentan que, ante cambios voluntarios respiración a respiración del $\mathrm{VC}_{\mathrm{LI}}-\mathrm{VC}_{\mathrm{LD}}$ a FR constante, la respuesta de la ASR es ambigua y presenta histéresis (Fig. 50). Más aún, que los efectos del $\mathrm{VC}_{\mathrm{LD}}$ son menos ambiguos que los del $\mathrm{VC}_{\mathrm{LI}}$. Esta discrepancia entre los hallazgos publicados y los propios podría explicarse por diferencias metodológicas: todos los estudios que han controlado al VC lo han hecho por etapas, es decir, manteniéndolo a un valor constante durante algunos minutos, mientras que en nuestras maniobras respiratorias los cambios fueron efectuados respiración a respiración, de manera que resultan más parecidos a los ajustes respiratorios en condiciones fisiológicas.

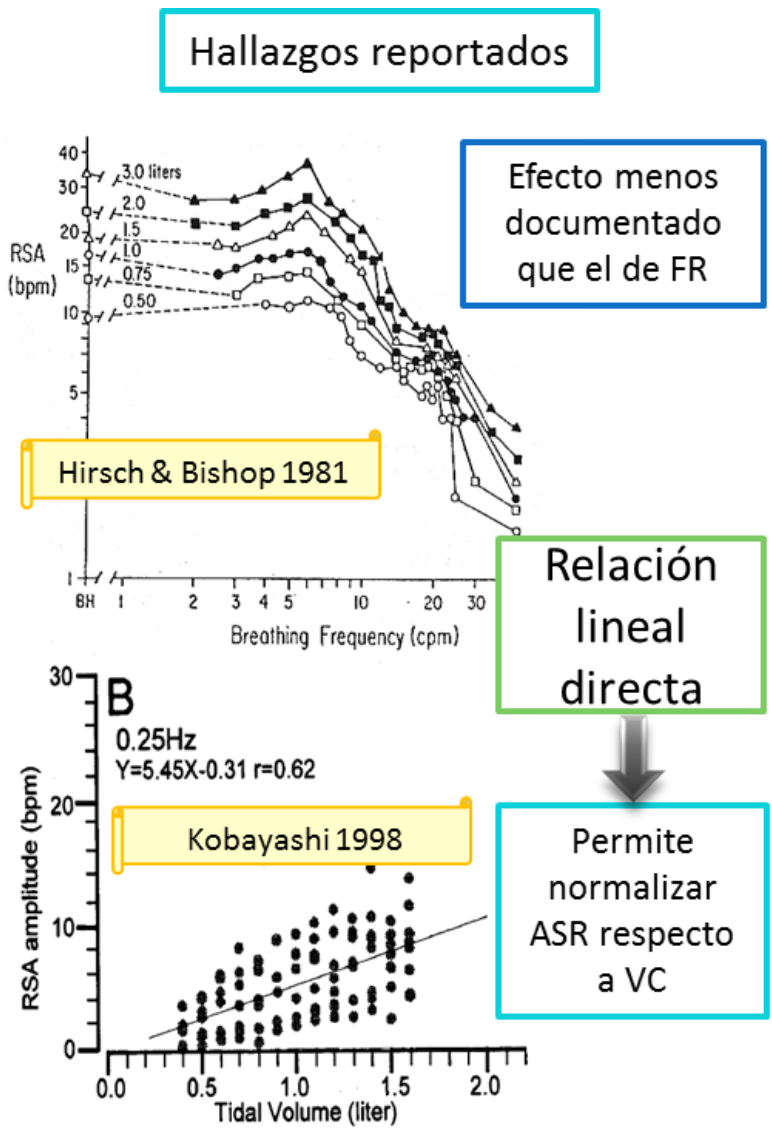

\section{VS.}
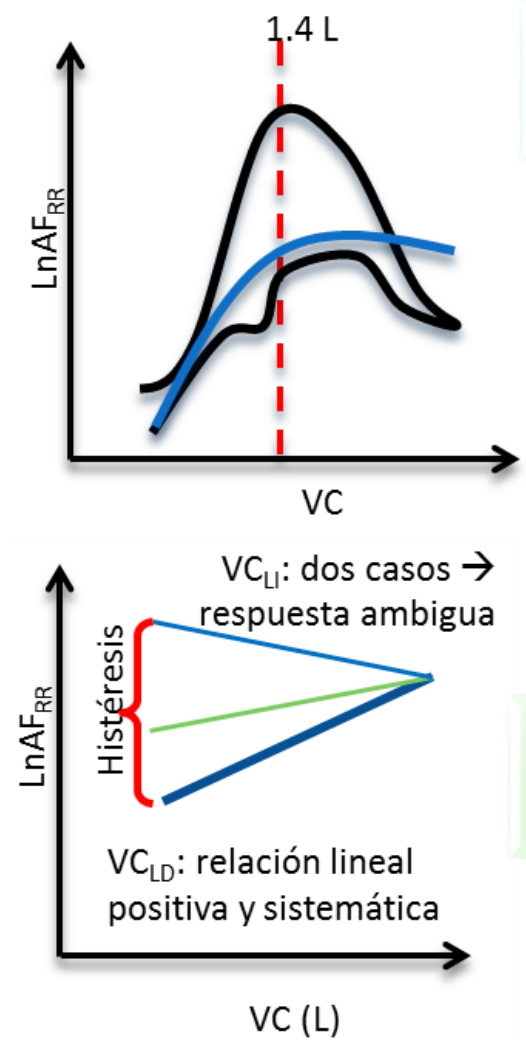

VC óptimo: fenómeno de umbral
Respuesta ambigua Histéresis

Fig. 50. En contraste con lo publicado, donde se asume que la amplitud del ASR aumenta proporcionalmente al VC, nuestros resultados documentan que para el $\mathrm{AF}_{\mathrm{RR}}$ hay un límite de $\mathrm{VC}$, un valor óptimo, más allá del cual su potencia decae. Además, los cambios de la potencia de $\operatorname{InAF}_{\mathrm{RR}}$ debidos a modificaciones voluntarias crecientes y decrecientes del VC son ambiguos y presentan histéresis.

\section{Efectos de la variación simultánea de FR y VC}

Hasta donde sabemos, este es el primer estudio en explorar bajo condiciones controladas los efectos sobre la magnitud de la ASR de la variación lineal, tanto de manera independiente como 
simultánea, de la FR y el VC. El principal efecto combinado consiste en una mayor atenuación de la potencia de $\ln \mathrm{AF}_{\mathrm{RR}}$ en relación al efecto independiente de cada variable respiratoria (Fig.51).

Más aún, la variación simultánea de ambas variables transforman sus efectos independientes sobre $\ln \mathrm{AF}_{\mathrm{RR}}$ en un patrón de respuesta sistemático: atenuación lineal con $\mathrm{VC}_{\mathrm{LI}}$ (pendiente negativa) e incremento lineal con $\mathrm{VC}_{\mathrm{LD}}$ (pendiente positiva), que presenta una histéresis mucho mayor que la originada por las variaciones independientes del VC. Esto indica que el efecto inhibitorio de $\mathrm{FR}_{\mathrm{LI}}$ sobre la potencia de $\ln \mathrm{AF}_{\mathrm{RR}}$ inicialmente predomina sobre los efectos del $\mathrm{VC}_{\mathrm{LI}} \mathrm{y}$ después se suma al efecto inhibitorio del $\mathrm{VC}_{\mathrm{LD}}$.

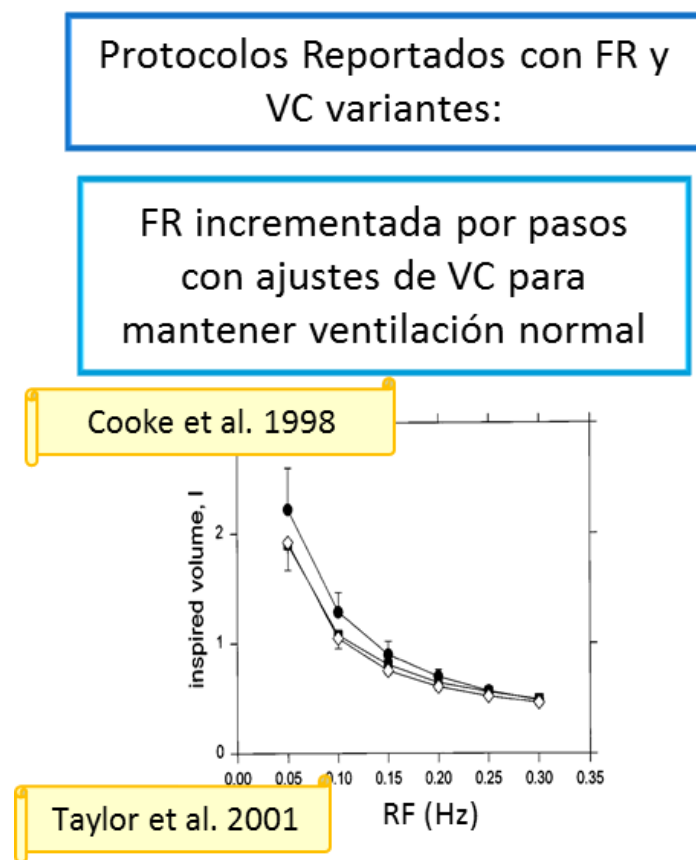

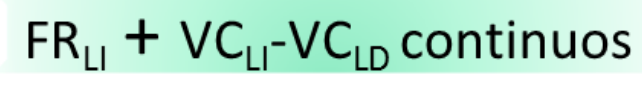
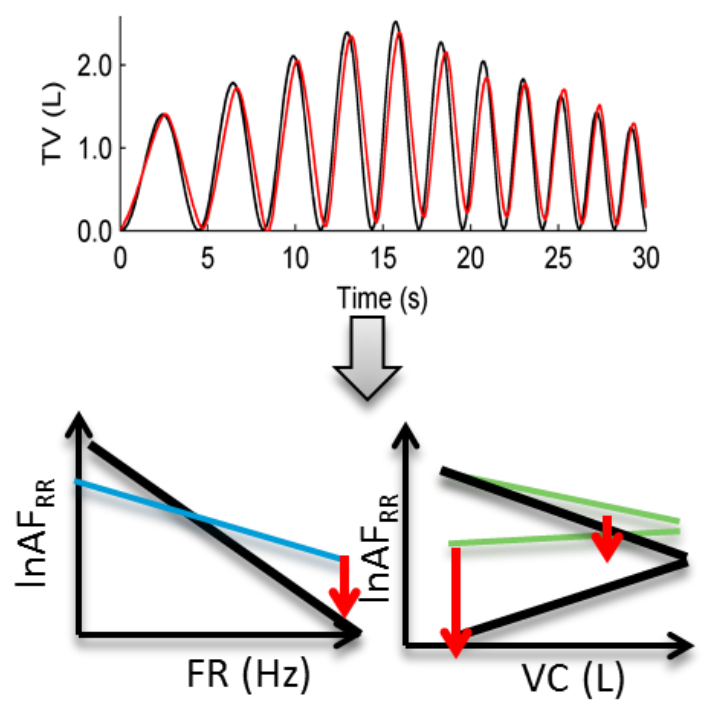

Fig. 51. Aunque en unos pocos artículos publicados se controlaron tanto la FR como el VC, su objetivo no fue estudiar qué efecto tenía su variación simultánea sobre la amplitud de la ASR. Mediante la estrategia metodológica de comparar los efectos independientes de cada variable con los efectos de las variables combinadas, encontramos que, durante la maniobra combinada, la ASR se atenúa más, su patrón de respuesta se hace más sistemático y presenta mayor histéresis.

\section{Efectos de ETCO2}

Todas las maniobras de control respiratorio voluntario empleadas en este estudio produjeron una hipocapnia ligera pero significativa, máxima en la maniobra combinada (Figs. 34, 39 y 43). Se ha documentado que los cambios en los niveles de $\mathrm{CO}_{2}$ afectan a la función cardiovascular. La hipocapnia ha sido asociada a incrementos en la frecuencia cardiaca y el gasto cardiaco, y a reducciones en la presión arterial, resistencia vascular sistémica y flujo sanguíneo cerebral 
(Burnum et al. 1954, Richardson et al. 1972, Henry et al. 1998, Ainslie y Duffin 2009). Sin embargo, también se ha reportado que la hipercapnia produce aceleración de la frecuencia cardiaca (Bristow et al. 1974). Los niveles de $\mathrm{CO}_{2}$ pueden influir en la amplitud de la ASR mediante mecanismos directos o indirectos. Hay evidencias que documentan las interacciones entre los quimio y los barorreceptores (Henry et al. 1998) y la posible participación de la actividad simpática en la reactividad cerebrovascular al $\mathrm{CO}_{2}$ (Peebles et al. 2012). No obstante, otros autores dudan que la hipocapnia moderada influya significativamente en el tono y la modulación de las salidas simpática y parasimpática (Pinna et al. 2006, Stankowski et al. 2013) y otros más han encontrado que la activación barorrefleja del simpático no tiene influencia sobre las respuestas cerebrovasculares al $\mathrm{CO}_{2}$ (LeMarbre et al. 2003). Con base en los reportes anteriores, se puede argüir que los efectos del nivel de $\mathrm{CO}_{2}$ sobre la actividad del SNA son pequeños, no son claros y su mecanismo mediador no ha sido dilucidado aún. Aún más, a pesar de que en este estudio la hipocapnia fue significativamente mayor en los hombres que en las mujeres, su esperado nivel vagolítico no se reflejó en un $\ln \mathrm{AF}_{\mathrm{RR}}$ significativamente menor en ellos (Tabla 2, Figs. 43 y 44). A pesar de todo, no podemos descartar completamente un efecto confundidor de la hipocapnia sobre los cambios en la amplitud de la ASR sin hacer pruebas manteniendo el $\mathrm{ETCO}_{2}$ constante.

\section{Diferencias entre sexos}

Estudios previos que han comparado los niveles basales de ASR entre hombres y mujeres o su respuesta a diferentes maniobras han arrojado resultados controversiales. En un estudio de gemelos idénticos, las mujeres presentaron mayor ASR que los hombres en condiciones de reposo. Pero en respuesta a la estimulación de la frente con frío, los hombres mostraron mayores incrementos en ASR y bradicardia que las mujeres (Ruiz et al. 2006). La respuesta de la ASR a la duplicación del VC basal con FR libre fue similar en ambos sexos (Sébert 1983). Además, el periodo cardiaco y la $\mathrm{AF}_{\mathrm{RR}}$ no fueron diferentes entre hombres y mujeres sin importar la condición respiratoria (Stark et al. 2000). Los últimos dos estudios concuerdan con la gran similitud de los efectos de nuestras maniobras sobre la actividad vagal de hombres y mujeres encontrada en el presente estudio, especialmente la maniobra combinada, la cual sistematizó la respuesta de la ASR (Figs. 43 a 45, Tablas 2 a 5). Incluso con una VP 20\% menor que la de 
los hombres, causada por una reducción del VC de magnitud equivalente, las mujeres lograron una inhibición vagal similar a la de los hombres.

\section{Efectos del CPA}

El sistema núcleos vagales-nodo SA integra las aferencias respiratorias y barorreflejas. Al mantener constante la influencia respiratoria gracias a la ejecución voluntaria de las maniobras diseñadas, los cambios en el $\ln \mathrm{AF}_{\mathrm{RR}}$ reflejan únicamente el efecto del CPA. La reducción del coeficiente de correlación puede explicarse por la aparición de una recuperación de actividad vagal resultante de los cambios dinámicos en la aferencia barorrefleja a consecuencia del cambio de postura (Figs. 39 y 40). La compleja dinámica de la presión arterial en respuesta al CPA explica la recuperación de $\ln \mathrm{AF}_{\mathrm{RR}}$ a los pocos segundos de iniciada la maniobra. Los resultados soportan que los requerimientos propuestos para el diseño del indicador vagal fueron satisfechos: toma en cuenta la influencia de las dos variables respiratorias, por su brevedad y facilidad de ejecución se puede superponer a otras maniobras, y la respuesta autonómica resultó manejable y comparable, inclusive entre hombres y mujeres, otorgando mayor robustez a la interpretación de los cambios de potencia del componente de $\mathrm{AF}_{\mathrm{RR}}$ como indicador vagal (Fig. 52). 


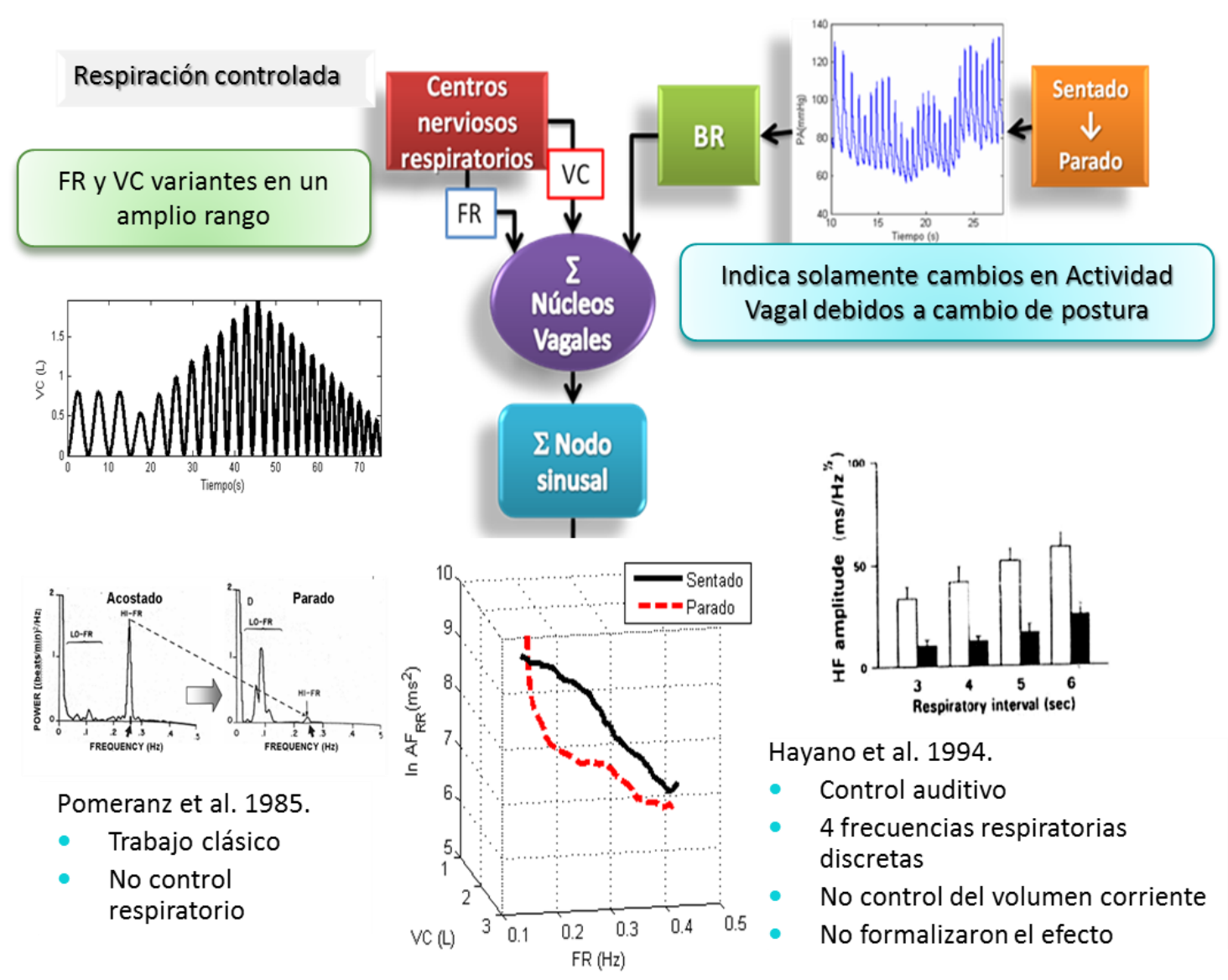

Fig. 52. El sistema núcleos vagales-nodo SA integra las aferencias de las variables respiratorias y barorreflejas. Gracias a las maniobras diseñadas, la influencia respiratoria se mantiene constante y los cambios en el InAF $F_{R R}$ reflejan únicamente el efecto del cambio de postura.

\section{Posibles mecanismos fisiológicos}

Cómo ejerce la respiración su influencia sobre las oscilaciones cardiacas es un tema largamente debatido y sólo parcialmente resuelto. El origen de la ASR ha sido explicado por los tres mecanismos siguientes (Larsen et al. 2010, Stankovski et al. 2013): (1) la influencia directa de las motoneuronas respiratorias centrales sobre las motoneuronas autonómico-cardiacas, (2) un efecto indirecto, mediado por el barorreflejo, de las fluctuaciones en la presión arterial inducidas por la respiración y (3) una respuesta cardiaca refleja al inflado mecánico de los pulmones (Fig. 53). 


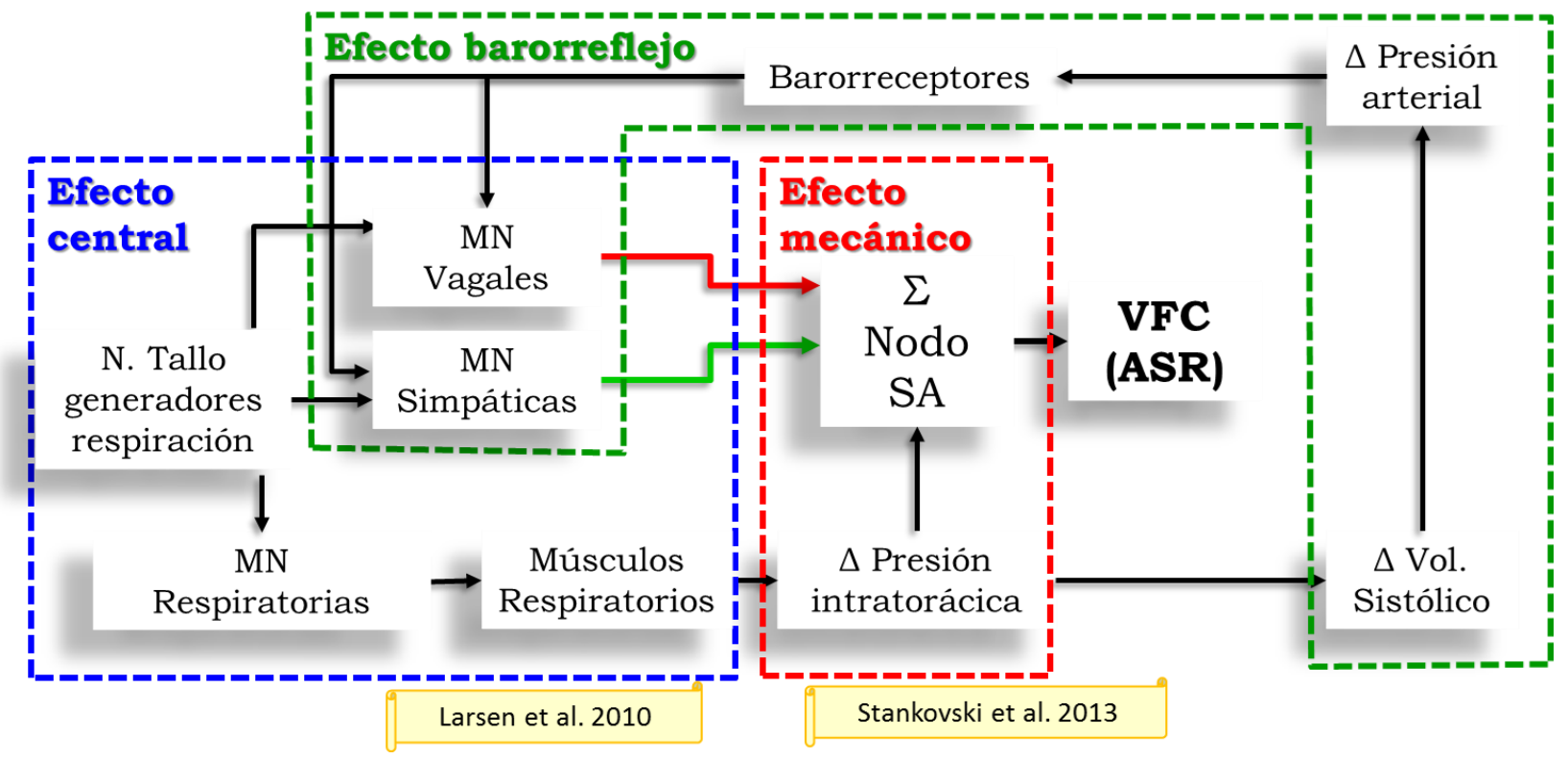

Fig. 53. Aún se debaten los mecanismos fisiológicos generadores de la ASR. Actualmente se acepta que es un fenómeno complejo, que resulta de la integración de al menos tres mecanismos: la influencia de la descarga de los núcleos del tallo generadores de la respiración espontánea sobre los núcleos autonómicos, denominado el mecanismo central; y dos efectos mecánicos de los cambios de presión intratorácica, uno directo debido a la deformación cíclica de la geometría del nodo SA y uno indirecto, mediado por el barorreflejo en respuesta a los cambios en el volumen sistólico causados por las modificaciones ventilatorias de la precarga y la poscarga.

La mayoría de los estudios acerca de la ASR se han enfocado más en la influencia respiratoria sobre la actividad vagal que sobre la simpática. Sin embargo, existen suficientes indicios publicados que apoyan que la actividad de los nervios simpáticos musculares es proporcional a la respiración (Badra et al. 2001), y que el bloqueo beta-adrenérgico incrementa la amplitud de la ASR, lo que en conjunto sugiere que la ASR no es mediada exclusivamente por la actividad vagal sino que también la actividad simpática participa, aunque el mecanismo preciso no ha sido dilucidado aún (Taylor et al. 2001).

Un estudio reciente documentó que los efectos de una maniobra respiratoria fueron ejercidos principalmente a través del mecanismo central y, en menor medida, por el barorreflejo. Así, incrementar la FR mediante las motoneuronas respiratorias causa una reducción sustancial en la actividad de las motoneuronas vagales y un débil incremento en la actividad de las motoneuronas simpáticas, afectando más a las oscilaciones que a los niveles medios de ambos sistemas (Stankovski et al. 2013). Análogamente, los presentes resultados pueden explicarse por los mecanismos generadores de ASR usuales, particularmente el central (Fig. 53): las maniobras de respiración controlada influyen de manera importante sobre la actividad de las motoneuronas 
respiratorias centrales, las cuales a su vez reducen la actividad de las motoneuronas vagales y, en menor medida, aumentan la actividad de las motoneuronas simpáticas. No obstante, pensamos que nuestros resultados son mejor explicados por un acoplamiento entre los núcleos somatomotores respiratorios y autonómicos (Fig. 54). La modificación voluntaria de la respiración durante el control del habla y la regulación volitiva de la respiración tienen en común que involucran la participación de áreas corticales motoras que controlan directamente a las motoneuronas medulares que inervan a los músculos respiratorios (Ackermann y Riecker 2010, Guz 1997, McKay et al. 2003). Sin embargo, el mecanismo mediante el cual el impulso voluntario de respirar puede influir sobre la ASR no es claro (Cooper et al. 2004), y su participación ha sido ignorada cuando se trata de explicar la respuesta autonómica a maniobras de control respiratorio voluntario. La ejecución precisa de las maniobras de respiración controlada guiadas visualmente involucra a múltiples estructuras cerebrales que corresponden, al menos parcialmente, al llamado comando central (CC), definido clásicamente como un sistema de anteroalimentación que implica la activación paralela de los centros de control somatomotor y autonómico-cardiovascular durante el ejercicio (Franke et al. 2000). Este concepto ha sido refinado y ampliado, de tal manera que actualmente se acepta que, en humanos conscientes, el CC está relacionado con el control motor voluntario (Vissing y Hjortsø 1996) y que consiste en la modulación de la función autonómica inducida por el esfuerzo percibido, aunque la activación somatomotora-autonómica paralela, sigue siendo el núcleo del concepto (Williamson et al. 2006).

Una posible explicación de nuestros resultados es que el CC integra información visual y propioceptiva compleja para llevar a cabo el complejo control somatomotor de los músculos respiratorios necesario para la ejecución de nuestras maniobras respiratorias. De manera paralela, simultánea y directa, el CC ajusta el tono y la modulación de las motoneuronas vagales al proveerlas de influencias inhibitorias y, en menor medida, proporcionar eferencias facilitatorias a las motoneuronas simpáticas (Fig. 54). 


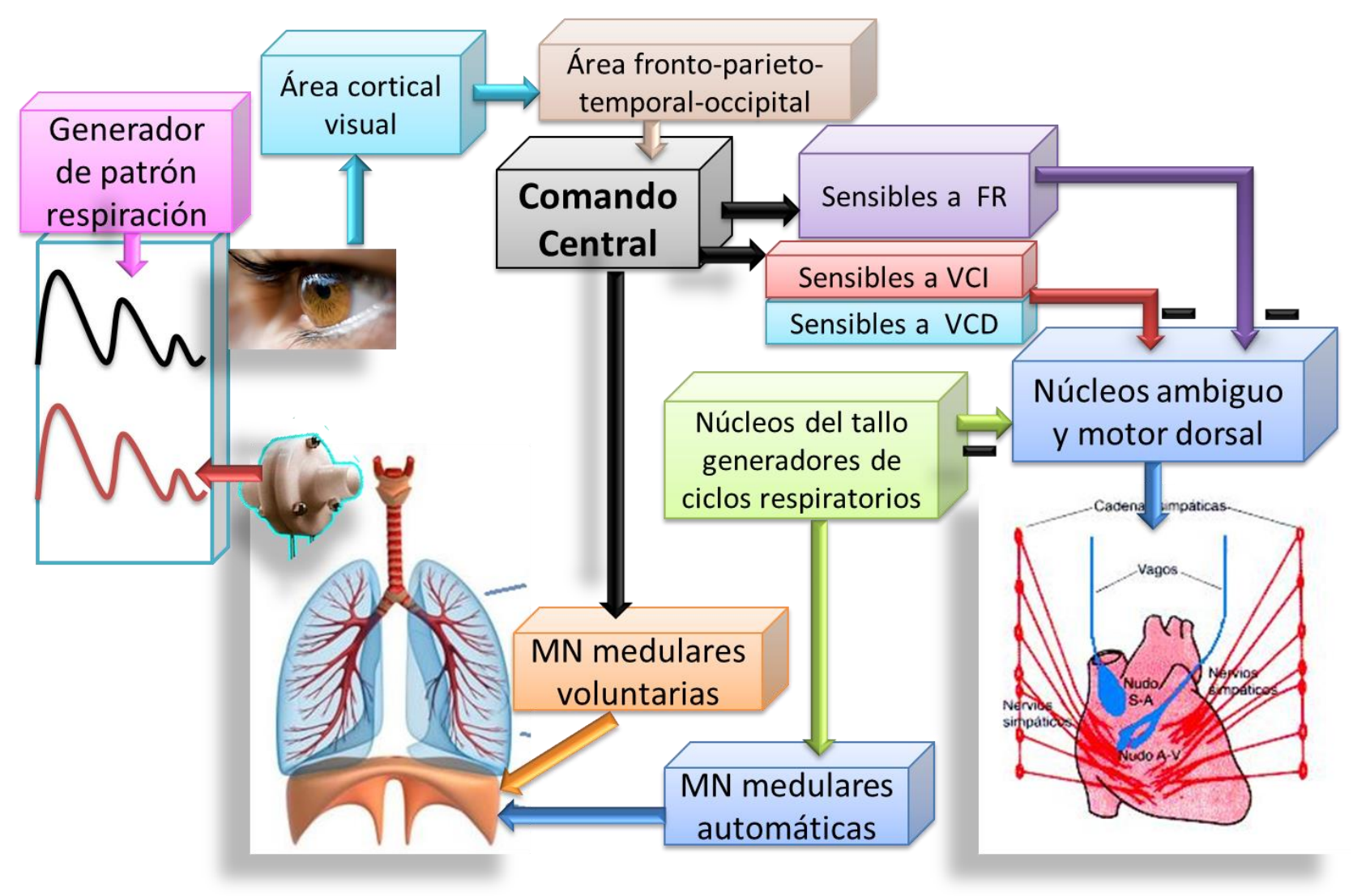

Fig. 54. Mecanismo fisiológico propuesto con base en nuestros resultados. Para lograr el control voluntario de los músculos respiratorios que permiten ajustar la respiración al patrón visualmente guiado, se involucran múltiples estructuras corticales que influyen de manera paralela a las motoneuronas medulares que controlan de manera voluntaria a los músculos respiratorios y a los núcleos originadores de los nervios autonómicos. Estos últimos parecen ser capaces integrar de manera independiente la influencia de la FR y del VC. Más aún, las diferencias entre las respuestas al $V_{C_{L I}}$ y al $V_{C_{L D}}$ podrían indicar que estos núcleos presentan diferentes sensibilidades a cada dirección de cambio de VC.

Análogamente al ejercicio desarrollado con los miembros, durante el cual ocurren incrementos concomitantes en la actividad del CC y la salida simpática, así como retiro vagal (Iellamo et al. 2001), la ejecución de nuestras maniobras respiratorias está asociada a incrementos no quimiorreflejos del VP y decrementos del $\mathrm{RR}_{\mathrm{m}}$, ambos proporcionales a la magnitud del esfuerzo de los músculos respiratorios (Figs. 34, 39 y 43). No obstante, la contribución de las motoneuronas respiratorias y del barorreflejo no debe ser pasada por alto, porque la combinación de estos mecanismos provee una explicación más plausible del complejo fenómeno de la ASR.

Durante la maniobra $\mathrm{FR}_{\mathrm{LI}}$ independiente, el incremento progresivo de la actividad del CC determina una inhibición proporcional del sistema núcleos vagales-nodo sinusal, como lo indica la fuerte correlación negativa encontrada ente $\ln \mathrm{AF}_{\mathrm{RR}}$ y $\mathrm{FR}_{\mathrm{LI}}$ (Fig. 45A, Tabla 4). Durante la maniobra $\mathrm{VC}_{\mathrm{LI}}-\mathrm{VC}_{\mathrm{LD}}$ independiente, a pesar de la actividad creciente-decreciente del $\mathrm{CC}$, la 
activación del sistema núcleos vagales-nodo sinusal no siempre cambia en proporción, como lo documentan las tres diferentes relaciones halladas entre $\ln \mathrm{AF}_{\mathrm{RR}}$ y $\mathrm{VC}_{\mathrm{LI}}-\mathrm{VC}_{\mathrm{LD}}$ (Fig. $45 \mathrm{~B}-\mathrm{C}$, Tabla 4). Éstas sugieren que los efectos de $\mathrm{VC}_{\mathrm{LI}}$ y $\mathrm{VC}_{\mathrm{LD}}$ son ejercidos mediante diferentes circuitos neurales del CC, lo que también podría explicar la histéresis observada. Pero una interpretación alterna podría ser que los núcleos autonómicos humanos son influidos por la memoria de las respiraciones pasadas, por lo cual la respuesta autonómica dependería más de la tendencia del cambio de las respiraciones previas que de la respiración actual, como ha sido propuesto para explicar los efectos diferentes de los cambios de FR de lento a rápido y de rápido a lento sobre la actividad autonómica (Stankovski et al. 2013). Aunque el esfuerzo respiratorio para la maniobra $\mathrm{VC}_{\mathrm{LI}}-\mathrm{VC}_{\mathrm{LD}}$ fue mayor que el de $\mathrm{FR}_{\mathrm{LI}}$, no determinó un efecto vagal mayor que este último (Tabla 3). Todo lo anterior sugiere que los núcleos vagales son menos sensibles a las variaciones del VC que de la FR (Fig. 54).

Durante la maniobra simultánea, en la parte correspondiente a $\mathrm{FR}_{\mathrm{LI}}-\mathrm{VC}_{\mathrm{LI}}$, la actividad del $\mathrm{CC}$ y el esfuerzo respiratorio son incrementados marcadamente, provocando una inhibición de la actividad del sistema núcleos vagales-nodo sinusal mayor a la causad por las maniobras independientes, que convierte las diferentes respuestas $\operatorname{lnAF}_{\mathrm{RR}}-\mathrm{VC}_{\mathrm{LI}}$ en una consistente relación con pendiente negativa (Fig. 45B, Tabla 5). Esta sistematización del efecto de $\mathrm{VC}_{\mathrm{LI}}$ refleja la mayor activación del sistema CC-núcleos vagales involucrada en el manejo de la variación conjunta de la FR y el VC. Este efecto es análogo a la disminución de la ASR en respuesta al incremento de la intensidad del esfuerzo y la VP durante el ejercicio dinámico (Arai et al. 1989). Durante la parte $\mathrm{FR}_{\mathrm{LI}}-\mathrm{VC}_{\mathrm{LD}}$ de la maniobra combinada, la activación del CC y el esfuerzo ventilatorio siguen elevados debido al incremento progresivo de la FR, produciendo una marcada inhibición de la actividad del sistema núcleos vagales-nodo sinusal, como lo documenta la reducción del intercepto de la relación $\ln \mathrm{AF}_{\mathrm{RR}}-\mathrm{VC}_{\mathrm{LD}}$ (Fig. 45B, Tabla 5).

La similitud entre los valores de las diferencias control-maniobra de $\ln \mathrm{AF}_{\mathrm{RR}}$ durante las maniobras independientes (Tabla 3) sugieren que $\mathrm{FR}_{\mathrm{LI}}$ y $\mathrm{VC}_{\mathrm{LI}}-\mathrm{VC}_{\mathrm{LD}}$ produjeron niveles de retiro vagal similares. Notablemente, el grado de inhibición vagal producido por la maniobra respiratoria simultánea, indicado por los valores de la diferencia control-maniobra en $\ln \mathrm{AF}_{\mathrm{RR}}$, es aproximadamente igual a la suma de las inhibiciones causadas por cada maniobra independiente (Fig. 44, Tabla 3). 
Para evaluar el tipo y grado de interferencia entre estímulos, en investigaciones de neurociencias se utiliza el II, definido como la razón entre la respuesta cortical que resulta de la aplicación simultánea de dos estímulos somatosensoriales dividida entre la suma de las respuestas producidas por cada estímulo aplicado por separado (Fig. 55). Si el II presenta un valor menor que uno, significa que los estímulos interactúan de manera oclusiva; si es mayor que uno, la interacción entre estímulos resulta en una potenciación de sus efectos. Un II de valor cercano a uno indica que los efectos de los estímulos no se interfieren mutuamente, lo que implica que las áreas corticales que responden a cada estímulo no se superponen y la integración neuronal de sus efectos es aditiva (Biermann et al. 1998, Hamada y Suzuki 2003).

En nuestro estudio, los cambios de amplitud de la ASR durante las maniobras donde FR y VC cambiaban simultáneamente fueron casi exactamente iguales a la suma de las respuestas a las maniobras independientes (Fig. 44), por lo que el II entre los efectos del FR y del VC sobre los valores medios del $R_{\mathrm{m}}$ y la potencia del $\ln \mathrm{AF}_{\mathrm{RR}}$ es muy cercano uno. Así, el comportamiento aditivo de los efectos vagales causados por la variación simultánea de FR y VC indica que sus aferencias sobre los núcleos autonómicos no se interfieren, sugiriendo que el procesamiento de los cambios en FR y VC lo llevan a cabo circuitos neurales vagales separados (Fig. 55). Aún más, si consideramos que el $\ln \mathrm{AF}_{\mathrm{RR}}$ presentó mayores correlaciones con $\mathrm{FR}_{\mathrm{LI}}$ que con $\mathrm{VC}_{\mathrm{LI}}$ o $\mathrm{VC}_{\mathrm{LD}}$, podemos suponer que la FR es el factor más influyente en la modulación de la actividad vagal. 

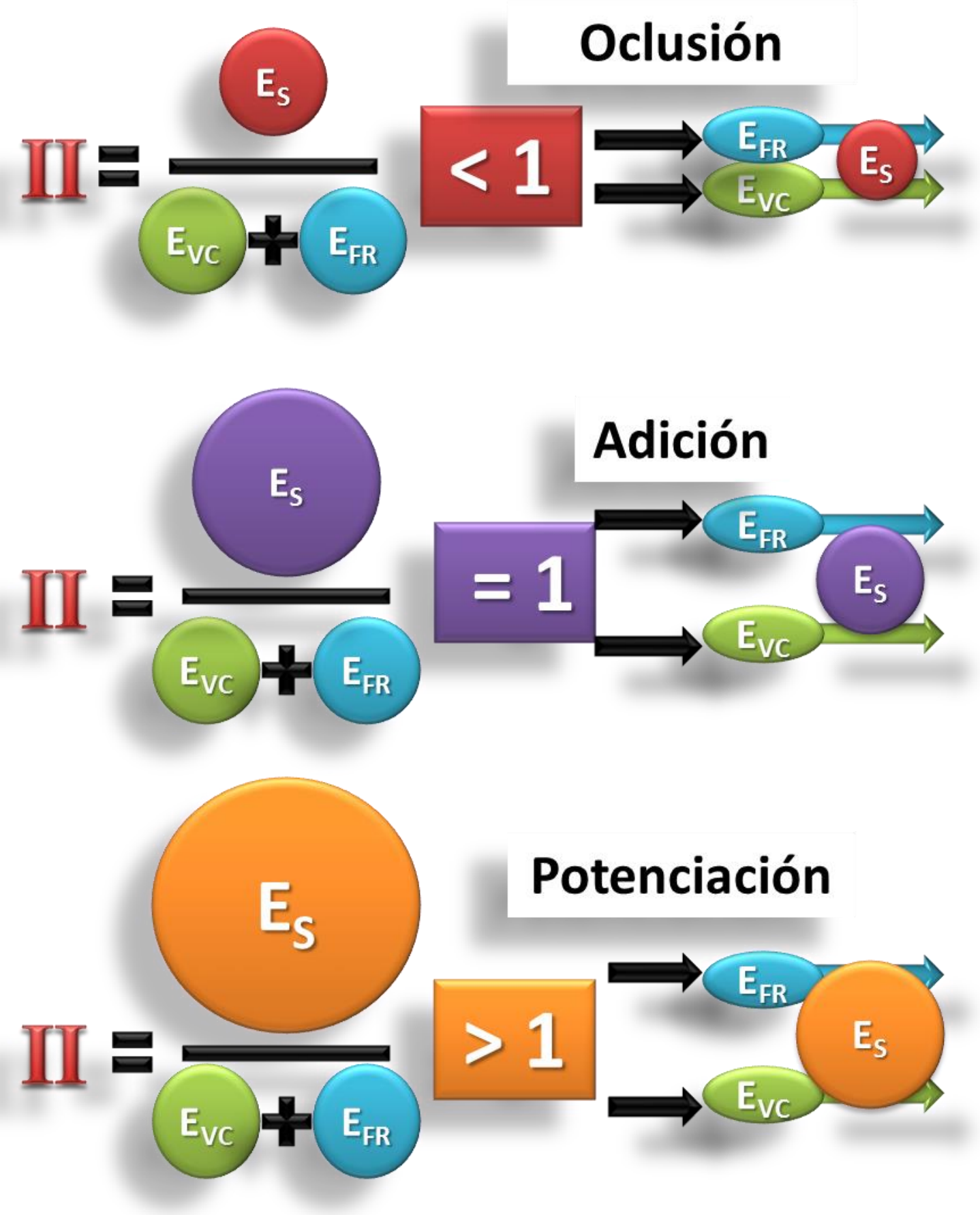

\section{Potenciación}

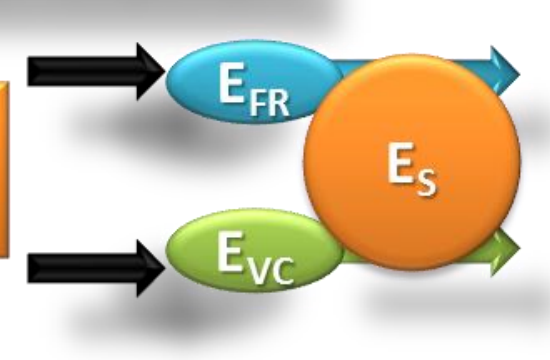

Fig. 55. El índice de interferencia (II) es utilizado en estudios de neurociencias (Biermann et al. 1998) para evaluar el tipo de interacción que ocurre entre dos estímulos con base en la respuesta que producen. Se obtiene calculando la razón entre el efecto que tiene la aplicación simultánea de los dos estímulos y la suma de los efectos de cada estímulo por separado. Si el II es menor que uno, los estímulos se ocluyen mutuamente; si es igual a uno, no se interfieren y la respuesta es aditiva, y si es mayor que uno, los estímulos se potencian. 
Las maniobras respiratorias utilizadas indujeron acortamientos significativos en el $\mathrm{RR}_{\mathrm{m}}$ debido a la reducción proporcional del tono vagal y/o al incremento del simpático (Figs. 34, 39, 44, Tabla 3). Este resultado concuerda con la reducción de los intervalos $\mathrm{RR}_{\mathrm{m}}$ producida al aumentar la $\mathrm{FR}$, recientemente publicada, y que fue explicada por una reducción del tono vagal (Stankovski et al. 2013). Pero nuestro hallazgo más notable es que la reducción de la media del $\mathrm{RR}_{\mathrm{m}}$ durante la maniobra simultánea es aproximadamente igual a la suma de las reducciones de $\mathrm{RR}_{\mathrm{m}}$ producidas por cada maniobra independiente (Fig. 44, Tabla 3). Por lo tanto, las reducciones de $\mathrm{RR}_{\mathrm{m}} \mathrm{y}$ $\ln A F_{R R}$ respaldan que las respuestas tanto de la modulación como del tono vagal son aditivas y están fuertemente asociadas entre ellas. Las reducciones de $\mathrm{RR}_{\mathrm{m}}$ observadas fueron proporcionales al esfuerzo respiratorio distintivo de cada maniobra respiratoria, indicado por el VP: a mayor esfuerzo, mayor reducción del $\mathrm{RR}_{\mathrm{m}}$ (Tabla 3). Se justifica especular que esta proporcionalidad es lograda por el CC mediante la reducción del tono vagal y/o el incremento del tono simpático, de manera similar a la explicación ofrecida para la relación encontrada entre la frecuencia cardiaca y la intensidad del ejercicio (Arai et al. 1989). Por lo tanto, bajo la condición de ejercicio respiratorio solo, los cambios en la $\mathrm{VP}$ y el $\mathrm{RR}_{\mathrm{m}}$ podrían ser utilizados como medida del grado de activación del CC-esfuerzo de músculos respiratorios, y de los ajustes concomitantes en el tono autonómico, respectivamente (Figs. 34, 39 y 44).

\section{Implicaciones}

Los hallazgos en torno a los efectos de las maniobras independientes $\mathrm{VC}_{\mathrm{LI}}-\mathrm{VC}_{\mathrm{LD}}$ sobre la amplitud de la ASR, v.g., el VC óptimo, las respuestas ambiguas y con histéresis que encontramos, tienen las siguientes implicaciones: 1) cuestionan a la noción comúnmente aceptada de que la amplitud de la ASR es lineal y directamente proporcional al VC, y por lo tanto a la validez de la práctica ampliamente extendida de normalizar a la ASR respecto al VC y 2) exhortan a controlar ambas variables respiratorias en estudios que emplean respiración controlada, que usualmente controlan la FR (Pinna et al. 2006) pero no el VC (Fig. 56). 


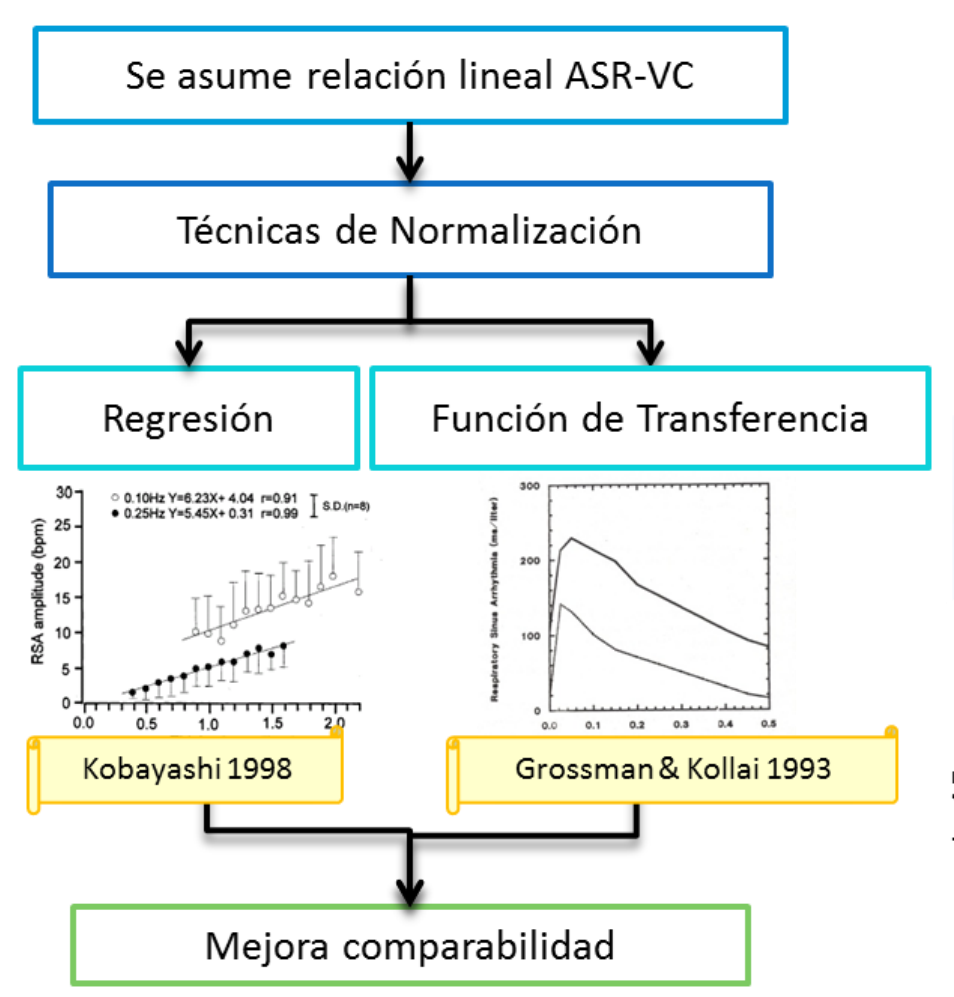

La mayoría de los estudios con

respiración controlada no controlan el VC

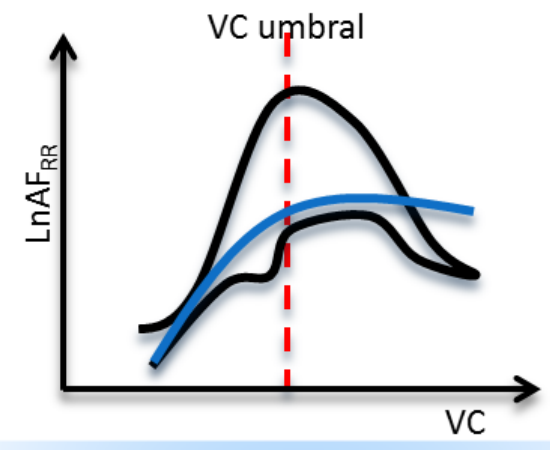

Efecto no lineal de VC sobre ASR $\rightarrow$ cuestiona validez de normalizar ASR respecto al VC

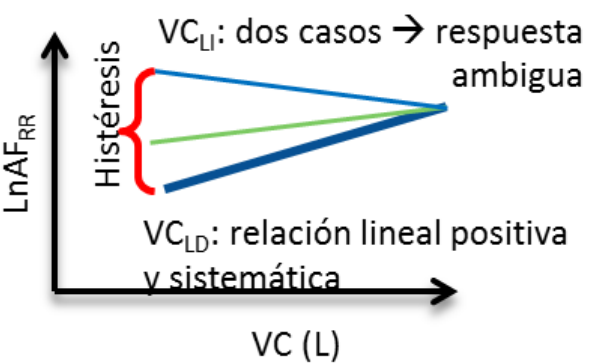

Por sus efectos ambiguos

$\rightarrow$ VC DEBE ser controlado experimentalmente

Fig. 56. Para mejorar su interpretabilidad como indicador autonómico, se han publicado diversas metodologías para normalizar a la amplitud de la ASR respecto al VC, todas ellas basadas en la asunción de que existe una proporcionalidad directa entre ambas ASR y VC. Los hallazgos de que la variación continua del VC en dirección creciente y luego decreciente produce respuestas ambiguas, con valores umbrales e histéresis cuestionan seriamente la validez de la normalización y encomia a que el VC sea controlado experimentalmente. 


\section{Perspectivas}

Los resultados del presente proyecto de investigación contribuyen al refinamiento del conocimiento acerca de la relación funcional entre los núcleos vagales-nodo SA y otros núcleos nerviosos que afectan su respuesta. Asimismo, plantean un buen número de preguntas por resolver:

- Examinar el desempeño del indicador vagal desarrollado en otras maniobras, como estimulación con frío en la cara, inclinación pasiva, estimulación con frío en la mano y ejercicio.

- Poner a prueba su aplicabilidad como tarea visual-motora, para la evaluación de la coordinación neuro-respiratoria en enfermos neurológicos.

- Examinar la ASR en respuesta a la aplicación del protocolo que implementamos en pacientes con neuropatía diabética o con insuficiencia cardiaca, dado que estos padecimientos han sido asociados con reducciones en la actividad vagal. Sería interesante evaluar: a) si el SNA de estos pacientes es todavía capaz de responder a variaciones continuas y lineales de FR y VC aplicadas de manera tanto independiente como combinada, b) si la modulación parasimpática presenta una reducción constante ante un amplio rango de valores de FR y VC o si existe alguna combinación de estas variables que la incremente, y c) si sus respuestas presentan valores umbrales de ambas variables respiratorias y cómo se comparan estos valores con respecto a individuos sanos.

- Investigar más a fondo los fenómenos de umbral, tanto en VC como en FR, que se presentan en las maniobras para evaluación fisiológica. En particular, la incorporación de la frecuencia cardiaca a la evaluación del aliasing cardiaco, ya sea como una tercera variable o como un factor de normalización de la FR, aportaría más información. También sería importante determinar el valor umbral de VC de cada sujeto y si guarda alguna relación con alguna variable espirométrica como la capacidad vital o los volúmenes inspiratorio o espiratorio de reserva.

- Explorar la existencia de histéresis en la consistente respuesta de la ASR a los cambios en la FR utilizando maniobras donde dicha variable respiratoria cambie de manera continua y lineal en dirección tanto creciente como decreciente. 
- Analizar cómo responden a las maniobras diseñadas otros indicadores de la ASR, como el valor pico-a-pico del tacograma o la raíz cuadrática media de las diferencias sucesivas (RMSSD por sus siglas en inglés Root Mean Square of Succesive Differences)

- Evaluar la coherencia tiempo-frecuencia entre la respiración y el tacograma.

- Explorar los efectos de las maniobras de control respiratorio sobre otras variables cardiovasculares, como la presión arterial, el volumen sistólico o el gasto cardiaco, a fin de obtener un panorama más completo que aporte más información sobre los posibles mecanismos generadores de la ASR y su contribución relativa.

- Confirmar y detallar la participación del CC en la generación de la ASR.

- Estudiar más a fondo los posibles efectos de la hipocapnia sobre la amplitud de la ASR, efectuando las mismas maniobras de respiración controlada pero ahora introduciendo $\mathrm{CO}_{2}$ a la boquilla del sujeto, a fin de mantener invariable el valor de la $\mathrm{ETCO}_{2}$ durante todo el protocolo. También se podría diseñar nuevas maniobras de control respiratorio que produjeran una VP constante.

- Analizar los efectos de la respiración sobre la fase de la modulación autonómica de la función cardiovascular. 


\section{Conclusiones}

La metodología desarrollada para esta investigación, consistente en el diseño y aplicación de maniobras de respiración controlada, rápidas, no fatigantes y con variaciones continuas y lineales de la FR y el VC, junto con el registro y análisis de la VFC en respuesta a estas maniobras mediante una herramienta apropiada para el estudio de señales no estacionarias, permitió documentar numerosos hallazgos originales, pertinentes y relevantes sobre la fisiología de la ASR (Fig. 57):

- La magnitud de la ASR cambia de manera similar en mujeres y hombres.

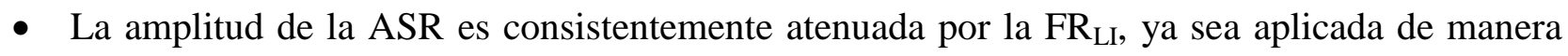
independiente o simultánea con cambios en el VC. Además, existe un valor límite de la FR para causar un efecto en la frecuencia del componente $\mathrm{AF}_{\mathrm{RR}}$.

- El componente de $\mathrm{AF}_{\mathrm{RR}}$ presenta respuestas ambiguas con histéresis a las variaciones de $\mathrm{VC}_{\mathrm{LI}^{-}}$ $\mathrm{VC}_{\mathrm{LD}}$, siendo los casos más frecuentes una relación lineal inversa con $\mathrm{VC}_{\mathrm{LI}} \mathrm{y}$ una relación lineal directa con $\mathrm{VC}_{\mathrm{LD}}$, con la ocurrencia de un valor de $\mathrm{VC}$ óptimo, más allá del cual la amplitud de la ASR no cambia o disminuye. Este efecto inconsistente de los cambios de VC en la amplitud de ASR encontrado cuestiona la práctica usual de normalizar la ASR con respecto a VC.

- La ASR se atenúa más cuando $\mathrm{FR}_{\mathrm{LI}}$ y $\mathrm{VC}_{\mathrm{LI}}-\mathrm{VC}_{\mathrm{LD}}$ se combinan, con una inhibición igual a la suma de las maniobras independientes. El efecto aditivo de la variación simultánea del VC y la FR sobre el componente de $\mathrm{AF}_{\mathrm{RR}}$ sugiere que los núcleos autonómicos tienen la capacidad de integrar los efectos de cada aferencia de manera independiente, aun cuando se aplican simultáneamente.

- Al realizar maniobras estimulantes autonómico-cardiacas junto con las maniobras de respiración controlada de corta duración se logró obtener un indicador vagal menos ambiguo que considera la influencia simultánea del VC y de la FR sobre la potencia de $\mathrm{AF}_{\mathrm{RR}}$.

Resulta importante resaltar que toda la información sobre las variables indicadoras del control autonómico-cardiovascular obtenida, analizada y reportada en la presente tesis se proporciona en un formato continuo y dinámico, que considera las variaciones temporales. Esto contrasta con la manera como normalmente se reportan las relaciones entre las variables autonómicocardiovasculares y respiratorias, que es mediante puntos aislados equivalentes al valor medio de la variable durante un cierto periodo de tiempo. 


\section{Metodología versátil y viable.}
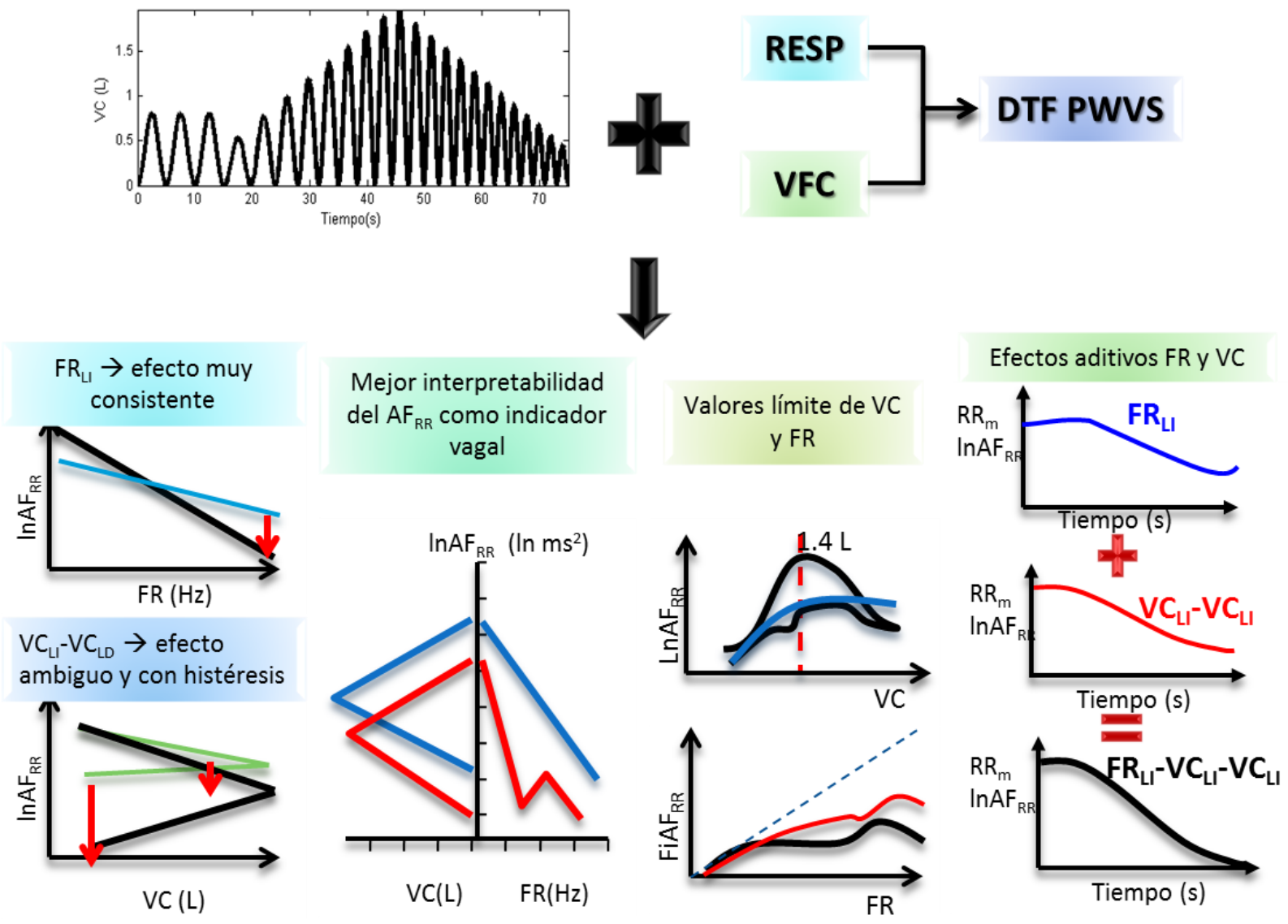

Fig. 57. La metodología desarrollada para la presente investigación redundó en múltiples hallazgos novedosos, originales y pertinentes respecto a la relación entre las variables respiratorias y la amplitud de la ASR. Una de las características más relevantes de estos hallazgos es que los estímulos fueron aplicados y las respuestas obtenidas y analizadas de manera continua, por lo que se puede estudiar la dinámica, es decir, la evolución temporal de las respuestas.

Todos estos hallazgos refuerzan la noción de que la respiración no es un artefacto, sino una influencia importante sobre la VFC, por lo que es indispensable el registro respiratorio en todo estudio de VFC (Brown et al. 1993).

Las importantes diferencias encontradas en la respuesta de la ASR a las variaciones respiración a respiración incrementales y decrementales del VC resaltan la importancia de que la ASR sea analizada en condiciones respiratorias estandarizadas, ya que su comportamiento puede verse afectado por diferencias metodológicas. 


\section{Referencias}

Ackermann H, Riecker A. The contribution(s) of the insula to speech production: a review of the clinical and functional imaging literature. Brain Struct Funct 214:419-433, 2010.

Ainslie PN, Duffin J. Integration of cerebrovascular $\mathrm{CO}_{2}$ reactivity and chemoreflex control of breathing: mechanisms of regulation, measurement, and interpretation. Am J Physiol Regul Integr Comp Physiol 296:R1473-R1495, 2009.

Arai Y, Saul JP, Albrecht P, Hartley LH, Lilly LS, Cohen RJ, Colucci WS. Modulation of cardiac autonomic activity during and immediately after exercise. Am J Physiol 256:H132-H141, 1989.

Badra LJ, Cooke WH, Hoag JB, Crossman AA, Kuusela TA, Tahvanainen KU, Eckberg DL. Respiratory modulation of human autonomic rhythms. Am J Physiol Heart Circ Physiol 280:H2674H2688, 2001.

Bailón R, Mainardi L, Laguna P. Time-frequency analysis of heart rate variability during stress testing using "a priori” information of respiratory frequency. Comput Cardiol 33:169-172, 2006.

Bianchi AM, Mainardi LT, Meloni C, Chierchia S, Cerutti S. Continuous monitoring of the sympathovagal balance through spectral analysis. IEEE Eng Med Biol Mag 16:64-73, 1997.

Biermann K, Schmitz F, Witte OW, Konczak J, Freund HJ, Schnitzler A. Interaction of finger representation in the human first somatosensory cortex: a neuromagnetic study. Neurosci Lett 251:13-16, 1998.

Bristow JD, Brown EB Jr., Cunningham DJ, Howson MG, Lee JR, Pickering TG, Sleight P. The effects of raising alveolar $\mathrm{PCO}_{2}$ and ventilation separately and together on the sensitivity and setting of the baroreceptor cardiodepressor reflex in man. J Physiol 243,401-425, 1974.

Brown TE, Beightol LA, Koh J, Eckberg DL. Important influence of respiration on human R-R interval power spectra is largely ignored. J Appl Physiol 75:2310-2317, 1993.

Burnum JF, Hickam JB, McIntosh HD. The effect of hypocapnia on arterial blood pressure. Circulation 9:89-95, 1954.

Cohen L. Time-Frequency analysis. Prentice Hall, USA, 1995.

Cooke WH, Cox JF, Diedrich AM, Taylor JA, Beightol LA, Ames JE, Hoag JB, Seidel H, Eckberg DL. Controlled breathing protocols probe human autonomic cardiovascular rhythms. Am J Physiol 274:H709-H718, 1998.

Cooper HE, Clutton-Brock TH, Parkes MJ. Contribution of the respiratory rhythm to sinus arrhythmia in normal unanesthetized subjects during positive-pressure mechanical hyperventilation. Am J Physiol Heart Circ Physiol 286:H402-H411, 2004. 
Cottin F, Médigue C, Leprêtre PM, Papelier Y, Koralsztein JP, Billat V. Heart rate variability during exercise performed below and above ventilatory threshold. Med Sci Sports Exerc 36:594-600, 2004.

Eckberg DL. Point: Respiratory Sinus Arrhythmia is due to a central mechanism. J Appl Physiol 106:1740-1742, 2009.

Eckberg DL. The human respiratory gate. J Physiol 548:339-352, 2003.

Franke WD, Boettger CF, McLean SP. Effects of varying central command and muscle mass on the cardiovascular responses to isometric exercise. Clin Physiol 20:380-387, 2000.

Gaitán MJ, Carrasco S, González CR, Yañez SO. Detección de onda R por diferenciación estadística del complejo QRS. IV Simposio del Departamento de Ciencias de la Salud, Univ. Autónoma Metropolitana Iztapalapa, 1998.

Gilbey MP, Jordan D, Richter DW, Spyer KM. Synaptic mechanisms involved in the inspiratory modulation of vagal cardio-inhibitory neurones in the cat. $J$ Physiol 356:65-78, 1984.

Grossman P, Taylor EW. Toward understanding respiratory sinus arrhythmia: Relations to cardiac vagal tone, evolution and biobehavioral functions. Biological Psychology 74:263-285, 2007.

Guz A. Brain, breathing and breathlessness. Respir Pysiol 109:197-204, 1997.

Hamada Y, Suzuki R. Hand posture modulates neuronal interaction in the primary somatosensory cortex of humans. Clin Neurophysiol 114:1689-1696, 2003.

Hankinson JL, Odencrantz JR, Fedan KB. Spirometric reference values from a sample of the general U.S. population. Am J Respir Crit Care Med 159:179-187, 1999.

Hayano J, Taylor JA, Yamada A, Mukai S, Hori R, Asakawa T, Yokoyama K, Watanabe Y, Takata K, Fujinami T. Continuous assessment of hemodynamic control by complex demodulation of cardiovascular variability. Am J Physiol 264:H1229-H1238, 1993.

Hayano J, Yasuma F, Okada A, Mukai S, Fujinami T. Respiratory Sinus Arrhythmia, a phenomenon improving pulmonary gas exchange and circulatory efficiency. Circulation 94:842-847, 1996.

Hayano J, Yasuma F. Hypothesis: respiratory sinus arrhythmia is an intrinsic resting function of cardiopulmonary system. Cardiovasc Res 58:1-9, 2003.

Henry RA, Lu IL, Beightol LA, Eckberg DL. Interactions between $\mathrm{CO}_{2}$ chemoreflexes and arterial baroreflexes. Am J Physiol 274:H2177-H2187, 1998.

Hirsch JA, Bishop B. Respiratory sinus arrhythmia in humans: how breathing pattern modulates heart rate. Am J Physiol 241: H620-H629, 1981.

Hrushensky W, Fader D, Schmitt O, Gilbertsen V. The respiratory sinus arrhythmia: a measure of cardiac age. Science, 224:1001-1004, 1984. 
Jasson S, Médigue C, Maison-Blanche P, Montano Nicola, et al. Instant power spectrum analysis of heart rate variability during orthostatic tilt using a time-frequency-domain method. Circulation 96:35213526, 1997.

Karemaker JM. Counterpoint: Respiratory Sinus Arrhythmia is due to the baroreflex mechanism. $J$ Appl Physiol 106:1742-1743, 2009.

Kim S, Euler D. Baroreflex sensitivity by complex demodulation of cardiovascular variability. Hypertension 29:1119-1125, 1997.

Kobayashi H. Normalization of respiratory sinus arrhythmia by factoring in tidal volume. Appl Human Sci 17:207-213, 1998.

Kollai M, Mizsei G. Respiratory sinus arrhythmia is a limited measure of cardiac parasympathetic measure in man. J Physiol 424:329-342, 1990.

Larsen PD, Tzeng YC, Sin PY, Galletly DC. Respiratory sinus arrhythmia in conscious humans during spontaneous respiration. Respir Physiol Neurobiol 174:111-118, 2010.

LeMarbre G, Stauber S, Khayat RN, Puleo DS, Skatrud JB, Morgan BJ. Baroreflex-induced sympathetic activation does not alter cerebrovascular $\mathrm{CO}_{2}$ responsiveness in humans. $J$ Physiol 551:609616, 2003.

Mainardi LT, Bianchi AM, Cerutti S. Time-frequency and time-varying analysis for assessing the dynamic responses of cardiovascular control. Crit Rev Biomed Eng 30:175-217, 2002.

Mainardi LT. On the quantification of heart rate variability spectral parameters using time-frequency and time-varying methods. Phil Trans R Soc A 367:255-275, 2009.

Malliani A, Pagani M, Lombardi F, Cerutti S. Cardiovascular neural regulation explored in the frequency domain. Circulation 84:482-492, 1991.

Malliani A, Pagani M, Montano N, Mela GS. Sympathovagal balance: a reappraisal. Circulation 98:2640-2643, 1998.

Mansier P, Médigue C, Charlotte N, Vermeiren C, Corabouef E, et al. Decreased heart rate variability in transgenic mice overexpressing atrial $\alpha_{1}$-adrenoreceptors. Am J Physiol 271:H1465-H1472, 1996.

Margaria R. Biomechanics and energetics of muscular exercise. Oxford University Press, 1976.

McKay LC, Evans KC, Frackowiak RS, Corfield DR. Neural correlates of voluntary breathing in humans. J Appl Physiol (1985) 95:1170-1178, 2003.

Meste O, Khaddoumi B, Blain G, Bermon S. Time-varying analysis methods and models for the respiratory and cardiac system coupling in graded exercise. IEEE Trans Biomed Eng 52:1921-1930, 2005 .

Monti A, Médigue C, Mangin L. Instantaneous parameter estimation in cardiovascular time series by harmonic and time-frequency analysis. IEEE Trans Biomed Eng 49:1547-1556, 2002. 
Mortola JP, Marghescu D, Siegrist-Johnstone R. Respiratory sinus arrhythmia in young men and women at different chest wall configurations. Clin Sci (Lond) 128:507-516, 2015.

Orini M, Bailón R, Mainardi LT, Laguna P, Flandrin P. Characterization of dynamic interactions between cardiovascular signals by time-frequency coherence. IEEE Trans Biomed Eng 59:663-673, 2012.

Pagani M, Lombardi F, Guzzetti S. Power spectral analysis of heart rate and arterial pressure variabilities as a marker of sympathovagal interaction in man and conscious dog. Circ Res 59: 178-193, 1986.

Parati G, Mancia G, Di Rienzo M, Castiglioni P. Point: cardiovascular variability is/is not an index of autonomic control of circulation. J Appl Physiol (1985) 101:676-678, 2006.

Parati G, Saul JP, Di Rienzo M, Mancia G. Spectral analysis of blood pressure and heart rate variability in evaluating cardiovascular regulation. Hypertension 25:1276-1286, 1995.

Peebles KC, Ball OG, MacRae BA, Horsman HM, Tzeng YC. Sympathetic regulation of the human cerebrovascular response to carbon dioxide. J Appl Physiol (1985) 113:700-706, 2012.

Pinna GD, Maestri R, La Rovere MT, Gobbi E, Fanfulla F. Effect of paced breathing on ventilatory and cardiovascular variability parameters during short-term investigations of autonomic function. Am $J$ Physiol Heart Circ Physiol 290:H424-H433, 2006.

Pomeranz B, Macaulay RJ, Caudill MA, Kutz I, Adam D, Gordon D, Kilborn KM, Barger AC, Shannon DC, Cohen RJ. Assessment of autonomic function in humans by heart rate spectral analysis. Am J Physiol 248: H151-H153, 1985.

Richardson DW, Kontos HA, Raper AJ, Patterson JL Jr. Systemic circulatory responses to hypocapnia in man. Am J Physiol 223:1308-1312, 1972.

Rocchetti M, Malfatto G, Lombardi F, Zaza A. Role of the input/output relation of sinoatrial myocytes in cholinergic modulation of heart rate variability. J Cardiovasc Electrophysiol 11:522-530, 2002.

Ruiz JM, Uchino BN, Smith TW. Hostility and sex differences in the magnitude, duration, and determinants of heart rate response to forehead cold pressor: parasympathetic aspects of risk. Int $J$ Psychophysiol 60:274-283, 2006.

Sasano N, Vesely AE, Hayano J, Sasano H, Somogoyi R, Preiss D, Miyasaka K, Katsuya H, Iscoe S, Fisher J. Direct effect of PaCO2 on respiratory sinus arrhythmia in conscious humans. Am J Physiol 282:H973-H976, 2002.

Saul JP, Berger RD, Chen MH, Cohen RJ. Transfer function analysis of autonomic regulation: II, respiratory sinus arrhythmia. Am J Physiol 256:H153-H161, 1989.

Saul JP, Cohen RJ. Respiratory Sinus Arrhythmia. En: Vagal Control of the heart: experimental basis and clinical implications. Editores: Levy M, Schwartz P. pp. 511-535. Futura Publishig, USA, 1994. 
Sébert P. Heart rate and breathing pattern: interactions and sex differences. Eur J Appl Physiol Occup Physiol 50:421-428, 1983.

Snieder H, van Doornen LJ, Boomsma DI, Thayer JF. Sex differences and heritability of two indices of heart rate dynamics: a twin study. Twin Res Hum Genet 10:364-372, 2007.

Stankovski T, Cooke WH, Rudas L, Stefanovska A, Eckberg DL. Time-frequency methods and voluntary ramped-frequency breathing: a powerful combination for exploration of human neurophysiological mechanisms. J Appl Physiol (1985) 115:1806-1821, 2013.

Stark R, Schienle A, Walter B, Vaitl D. Effects of paced respiration on heart period and heart period variability. Psychophysiology 37:302-309, 2000.

Tarvainen MP, Ranta-Aho PO, Karjalainen PA. An advanced detrending method with application to HRV analysis. IEEE Trans Biomed Eng 49:172-175, 2002.

Task Force of the European Society of Cardiology and the North American Society of Pacing and Electrophysiology. Heart rate variability, standards of measurement, physiological interpretation and clinical use. Circulation 93:1043-1065, 1996.

Taylor JA, Myers CW, Halliwill JR, Seidel H, Eckberg DL. Sympathetic restraint of respiratory sinus arrhythmia: implications for vagal cardiac tone assessment in humans. Am J Physiol Heart Circ Physiol 280:H2804-H2814, 2001.

Tzeng YC, Sin PY, Galletly DC. Human sinus arrhythmia: inconsistencies of a teleological hypothesis. Am J Physiol Heart Circ Physiol 296: H65-H70, 2009.

Vissing SF, Hjortsø EM. Central motor command activates sympathetic outflow to the cutaneous circulation in humans. J Physiol 492:931-939, 1996.

Williamson JW, Fadel PJ, Mitchell JH. New insights into central cardiovascular control during exercise in humans: a central command update. Exp Physiol 91:51-58, 2006.

Witte H, Zwiener U, Rother M, Glaser S. Evidence of a previously undescribed form of respiratory sinus arrhythmia (RSA)-the physiological manifestation of 'cardiac aliasing'. Eur J Physiol 412:442-444, 1988.

Yasuma F, Hayano J. Respiratory sinus arrhythmia: why does the heartbeat synchronize with respiratory rhythm? Chest 125:683-690, 2004. 


\title{
Anexo 1. Consentimiento Informado
}

\author{
$\Delta$ al \\ UNIVERSIDAD AUTONOMA METROPOLITANA \\ Laboratorio de Fisiología Médica
}

\section{Consentimiento Informado para participar como voluntario en un protocolo de investigación en Fisiología Médica}

Nombre del Investigador Principal: Dr. Salvador Carrasco Sosa

Dirección del Sitio de Investigación Laboratorio de Fisiología Médica, EdificioS, cubículo 353. Universidad

Autónoma Metropolitana, Unidad Iztapalapa. Av. San Rafael Atlixco No. 186, Col. Vicentina, 09340 México, D.F. Teléfono: 58046495. E-mail:scas@xanum.uam.mx

Por medio de la presente ACEPTO participar como voluntario en el protocolo de investigación titulado "Efectos de la variación simultánea de la frecuencia respiratoria y el volumen corriente sobre la actividad autonómica cardiovascular", cuyo objetivo es: generar conocimiento original y relevante sobre la interrelación funcional de la respiración controlada y el control autonómico cardiovascular, con énfasis en la amplitud de la Arritmia Sinusal Respiratoria (ASR), mediante el análisis de las respuestas de la frecuencia cardiaca a la estimulación de respirar cambiando voluntariamente la cantidad de respiraciones por minuto y la cantidad de aire en cada respiración.

Se me ha explicado que mi participación en este estudio consistirá en:

Visitar tres veces el Laboratorio de Fisiología Médica para el registro no invasivo de variables cardiovasculares y respiratorias mientras realizo diferentes maniobras de respiración controlada siguiendo las indicaciones de los investigadores, de un minuto o dos minutos y medio de duración. Esto implica que me colocarán tres electrodos adhesivos sobre la piel del tórax, deberé respirar a través de una boquilla y me pondrán una pinza que evitará que respire por la nariz. Cada visita durará entre 30 y 45 minutos.

Declaro que se me ha informado ampliamente sobre los posibles riesgos y molestias derivados de mi participación voluntaria en este estudio, los cuales son:

Ligeras molestias relacionadas con la colocación y/o retiro de los electrodos, el respirar por la boca en una boquilla con la nariz tapada o la restricción de movilidad general que implica la conexión de los sensores al equipo de medición y registro. Una leve posibilidad de presentar síntomas pasajeros relacionados con hiperventilación y/o el cambio de postura como: mareo, dolor de cabeza o pérdida del conocimiento. Si esto llegara a ocurrir, se interrumpirá inmediatamente el estudio y se me proporcionará la atención médica necesaria.

Declaro que se me ha dado oportunidad de hacer todas las preguntas que he querido y se han aclarado todas mis dudas, y que los investigadores se han comprometido a responder cualquier pregunta que pueda surgir en cualquier momento. Conservo el derecho pleno de retirarme del estudio en cualquier momento sin explicaciones. Se me ha garantizado el anonimato en todas las presentaciones y publicaciones que se deriven de este estudio y que toda la información personal recabada será manejada confidencialmente. Los investigadores se han comprometido a comunicarme toda la información derivada del estudio, aunque pudiera hacerme cambiar de parecer respecto a seguir participando.

Nombre del participante

Firma del Participante

Fecha de la firma

Nombre de quien obtiene el Consentimiento

Firma

$\overline{\text { Fecha de la firmal }}$

UNIDADIZTAPALAPA

Av. San Rafael Atlixco No. 186, Col. Vicentina, 09340 México, D.F. 


\section{Anexo 2. Publicaciones derivadas del proyecto}

Guillén-Mandujano A, Carrasco-Sosa S. Interactive Effects of Simultaneously Varying Respiratory Frequency and Tidal Volume on Respiratory Sinus Arrhythmia. Computing in Cardiology 39:165-168, 2012.

Guillén-Mandujano A, Carrasco-Sosa S. Additive effect of simultaneously varying respiratory frequency and tidal volume on respiratory sinus arrhythmia. Auton Neurosci. 186:69-76, 2014. 